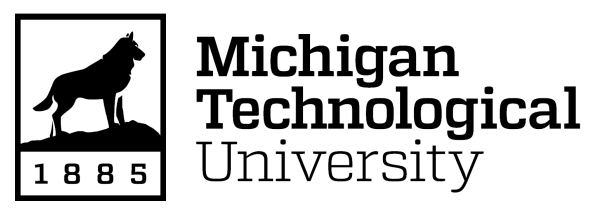

Michigan Technological University Digital Commons @ Michigan Tech

Dissertations, Master's Theses and Master's Reports

2017

Electrospinning Novel Aligned Polymer Fiber Structures for Use in Neural Tissue Engineering

Rachel Martin

Michigan Technological University, rachelm@mtu.edu

Copyright 2017 Rachel Martin

Recommended Citation

Martin, Rachel, "Electrospinning Novel Aligned Polymer Fiber Structures for Use in Neural Tissue Engineering", Open Access Dissertation, Michigan Technological University, 2017.

https://doi.org/10.37099/mtu.dc.etdr/521

Follow this and additional works at: https://digitalcommons.mtu.edu/etdr

Part of the Biology and Biomimetic Materials Commons, and the Nanoscience and Nanotechnology Commons 


\title{
ELECTROSPINNING NOVEL ALIGNED \\ POLYMER FIBER STRUCTURES FOR USE IN NEURAL TISSUE ENGINEERING
}

\author{
By \\ Rachel Ann Martin \\ A DISSERTATION \\ Submitted in partial fulfillment of the requirements for the degree of \\ DOCTOR OF PHILOSOPHY \\ In Chemical Engineering \\ MICHIGAN TECHNOLOGICAL UNIVERSITY \\ 2017 \\ (C) 2017 Rachel Ann Martin
}


This dissertation has been approved in partial fulfillment of the requirements for the Degree of DOCTOR OF PHILOSOPHY in Chemical Engineering.

Department of Chemical Engineering

$\begin{aligned} \text { Dissertation Co-Advisor: } & \text { Dr. Michael E. Mullins } \\ \text { Dissertation Co-Advisor: } & \text { Dr. Feng Zhou } \\ \text { Committee Member: } & \text { Dr. Caryn Heldt } \\ \text { Committee Member: } & \text { Dr. Jeremy Goldman } \\ \text { Department Chair: } & \text { Dr. Pradeep Agrawal }\end{aligned}$




\section{Table of Contents}

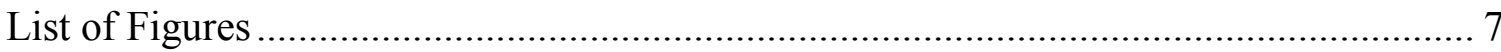

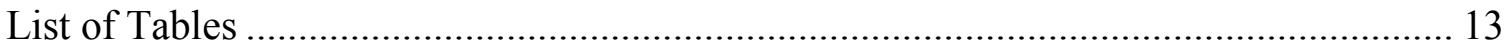

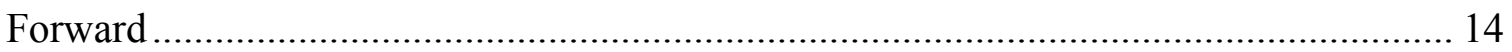

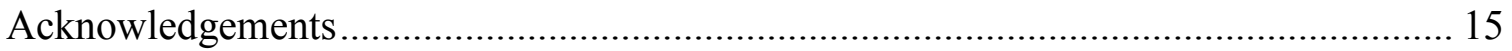

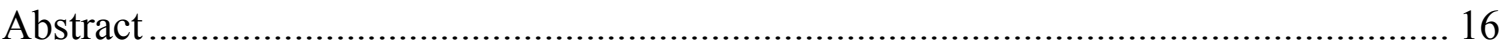

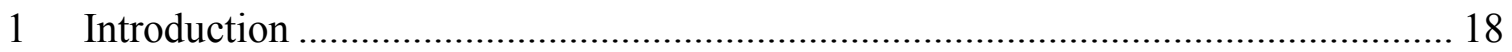

1.1 Motivation and Organization of Dissertation ................................................. 18

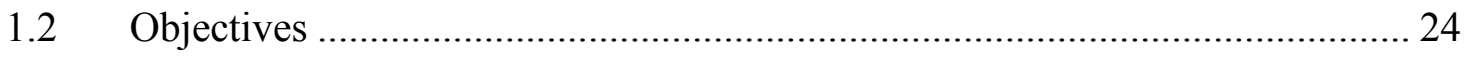

2 Electrospinning Techniques for Use in Neural Tissue Engineering ......................... 27

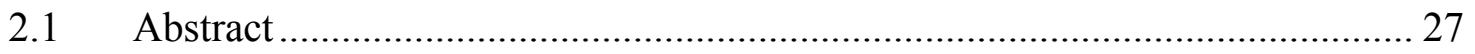

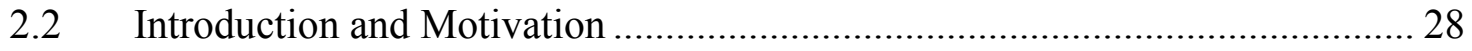

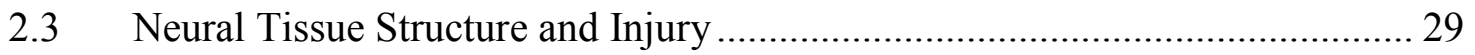

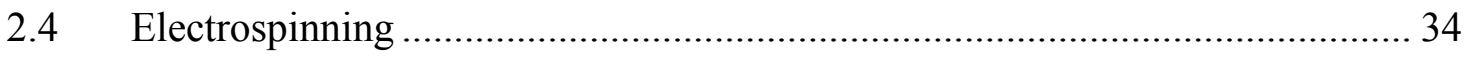

2.5 Applications of Electrospun Fibers .............................................................. 40

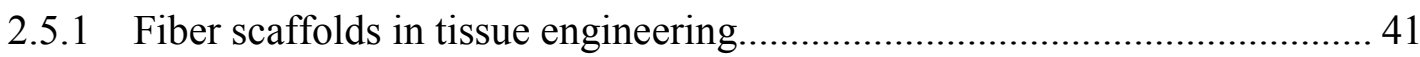

2.5.2 Aligned fiber scaffolds for neural tissue repair.......................................... 42

2.5.3 Aligned scaffolds in other tissue engineering applications........................... 48

2.5.4 Alternative polymers and blends for electrospun neural scaffolds ............... 48

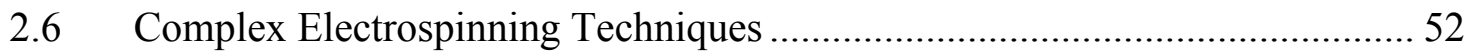

2.6.1 Coaxial fibers for polymer scaffolds in tissue engineering ......................... 52 
2.6.2 Application of conducting polymer fibers in scaffolds for neural tissue

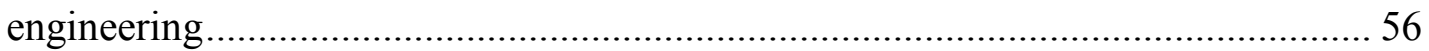

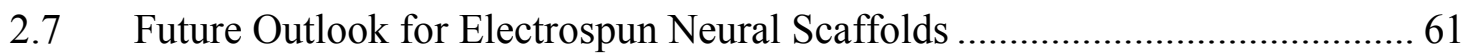

3 Electrospinning Aligned, Free-Standing PLLA Microfiber Scaffolds on Elevated

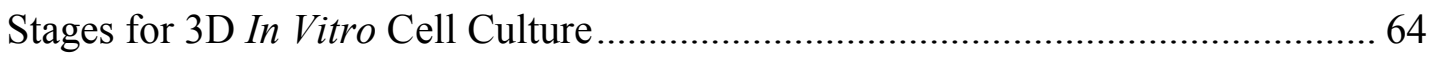

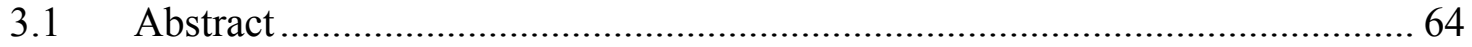

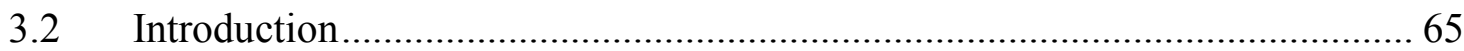

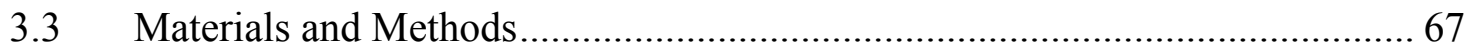

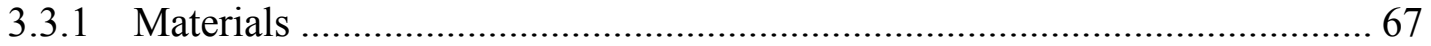

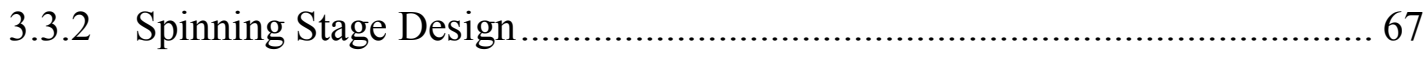

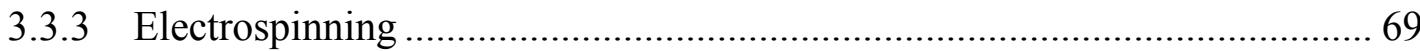

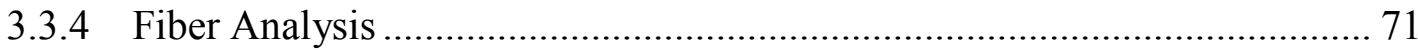

3.3.5 Dorsal Root Ganglia (DRG) Isolation and Culture................................... 72

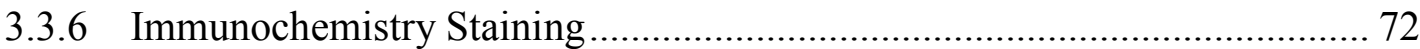

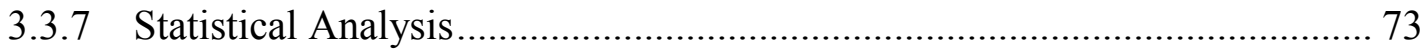

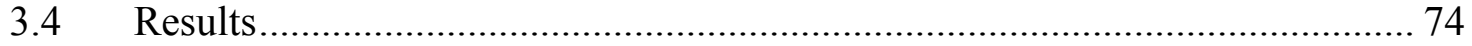

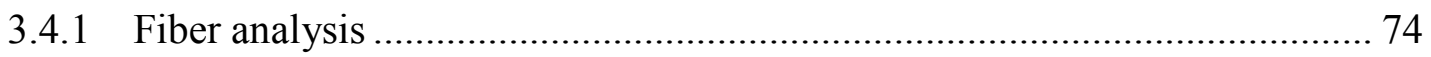

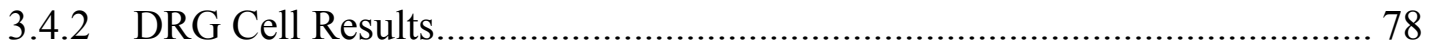

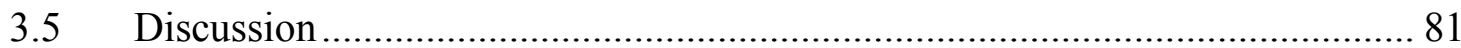

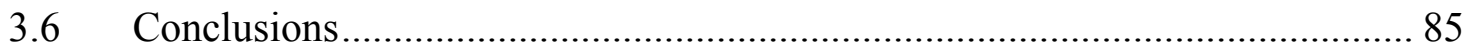

4 Formation of Aligned Core/Sheath Microfiber Scaffolds with a poly-L-lactic acid (PLLA) Sheath and a Conductive poly(3,4-ethylenedioxythiophene) (PEDOT)

Core 


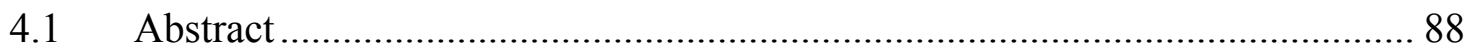

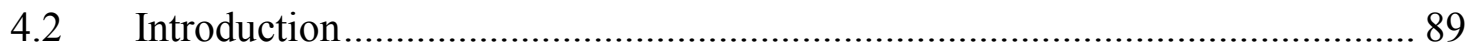

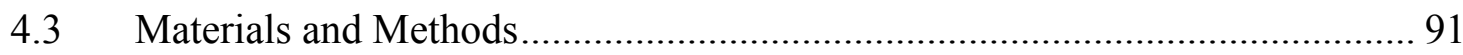

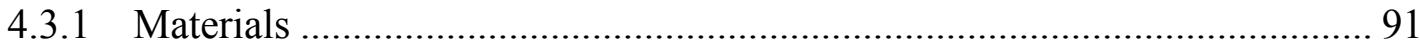

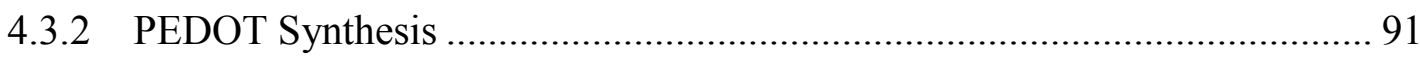

4.3.3 Coaxial Electrospinning Apparatus Design ................................................ 93

4.3.4 Testing of the Sheath Solution................................................................... 97

4.3.5 Preparation of the Core Solution/Suspension .............................................. 98

4.3.6 Coaxial Electrospinning Procedure............................................................ 99

4.3.7 Fluorescence Optical Imaging and FTIR Fiber Analysis and

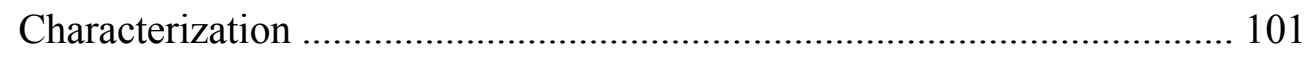

4.3.8 Field Emission Scanning Electron Microscopy Fiber Analysis and

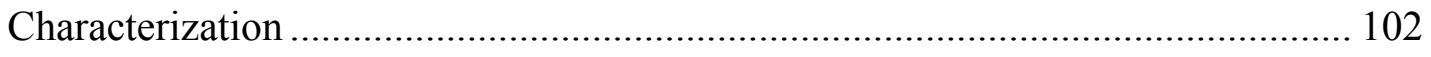

4.3.9 Mechanical Testing of Coaxial Microfibers ………….............................. 103

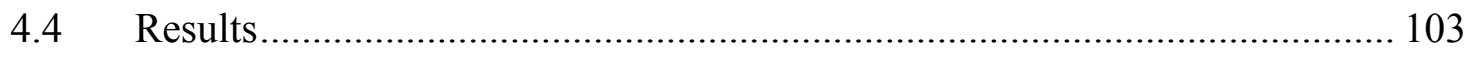

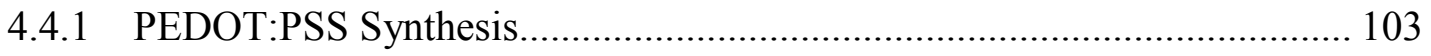

4.4.2 Viscosity and Flow Rate Analysis ........................................................... 105

4.4.3 Coaxial Fiber Characterization ................................................................ 109

4.4.4 Chemical and Mechanical Property Analysis ............................................. 115

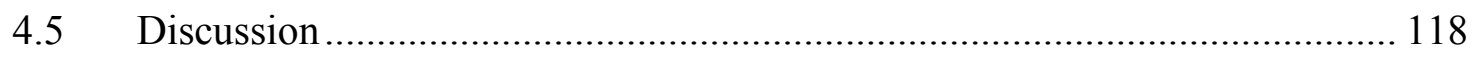

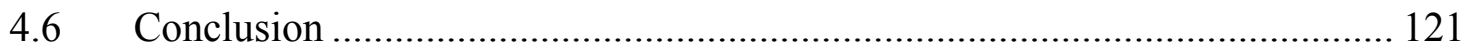

5 In Vitro Chick Dorsal Root Ganglia Studies on PEDOT/PLLA Core/Sheath

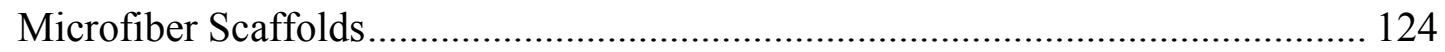




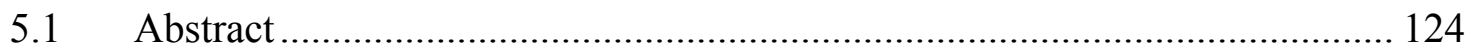

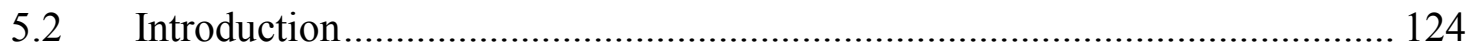

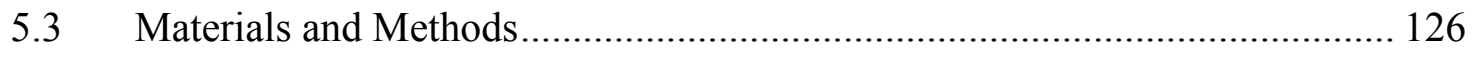

5.3.1 Coaxial Fiber Formation …………………............................................. 126

5.3.2 Dorsal Root Ganglia (DRG) Isolation and Culture.................................... 126

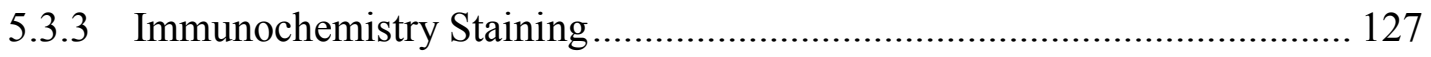

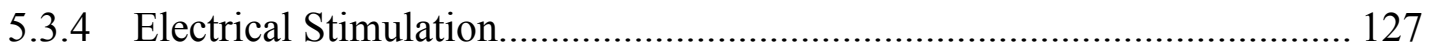

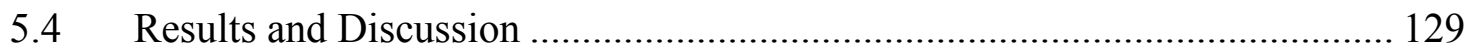

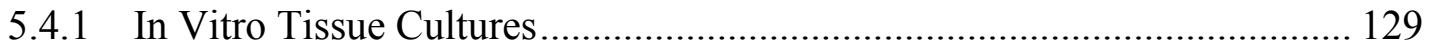

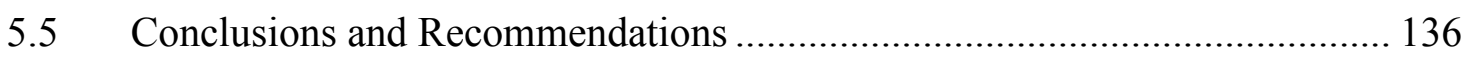

6 Overall Conclusions and Recommendations for Future Work............................... 140

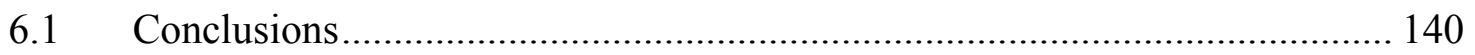

6.2 Recommendations for Future Work.......................................................... 145

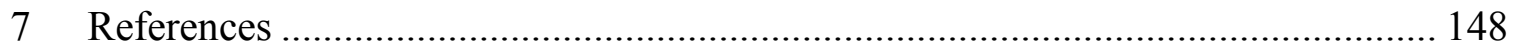

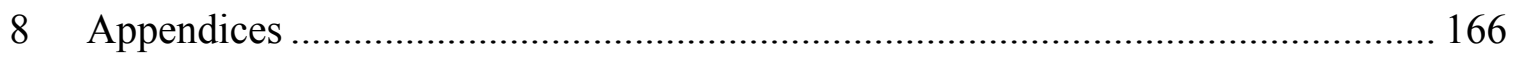

8.1 2015 MRS Conference Proceedings: Electrospinning 3D Scaffolds for use in

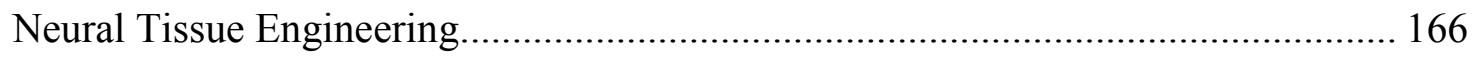

8.2 Additional Optical Microscopy and FE-SEM Images Showing Core-Sheath

Structure

8.3 Copyright Clearance for Images used in Chapter 2 …………..................... 184 


\section{List of Figures}

Figure 2-1: Cellular response to injury to the CNS.

Figure 2-2: Electrospinning apparatus for collection on a flat, stationary collector and a rotating wheel collector.

Figure 2-3: Poly-L-lactic acid nanofibers (A) random fibers on a station collector (B) aligned fibers on a rotating collector ...... 39

Figure 2-4: Chick DRG cell cultures. (A)Cell cultured without topographical cues (B)Cell cultured on aligned fiber scaffold 44

Figure 2-5: A-C: SEM images of various alignment of electrospun PLLA fibers. D-F: FFT images from the SEM images in A-C. G-I: Neurite outgrowth from DRG cells. J-L: FFT images from the images in G-I. Reused with permission from Wiley (Appendix 8.3)

Figure 2-6:(A) Highly aligned electrospun PLLA nanofibrous mat. (B) Stage E9 chick dorsal root ganglia (DRG) showing axonal extension along fiber alignment. The aligned fibers aided in highly extensive axonal outgrowth. Reused with permission from IOP Science (Appendix 8.3)

Figure 2-7: (A) Micrograph of DRG neurite outgrowth field on random polymer fiber mat with PCL-PPY coaxial fibers. (B) Aligned PCL-PPY coaxial fibers. (C) Micrograph of DRG neurite outgrowth field on aligned PCL-PPY coaxial fibers. Reused with permission from Wiley (Appendix 8.3) 61

Figure 3-1: 3D-printed electrospinning stages 69 
Figure 3-2: Taylor's cone observed. right-hand image: captured during electrospinning. left-hand image: drawing. 71

Figure 3-3: Optical microscope images of electrospun PLLA fibers. Spinning time for samples A and B was $32 \mathrm{~min}$ with a voltage of $17 \mathrm{KV}$ and a flow rate of $0.9 \mathrm{~mL} / \mathrm{hr}$. The fibers were spun on 3D stages with $3 \mathrm{~mm}$ columns. C and D show fibers spun on flat coverslips at a spinning time of $23 \mathrm{~min}$ with a voltage of $17 \mathrm{KV}$ at a flow rate of $1.05 \mathrm{~mL} / \mathrm{hr}$ 75

Figure 3-4: Plotted number of fibers at certain degrees from alignment. Top: $23 \mathrm{~min}$ Middle: 32min Bottom: coverslip 76

Figure 3-5: Frequency plots from alignment data. Top: 23min Middle: 32min Bottom: coverslip 77

Figure 3-6 Immunochemical staining of 5-day chick DRG cultures on (A) coverslip (B) fibers. Without the topographical cues provided by the aligned fiber scaffolds, the axons become entangled in a wreath-like structure around the center of the cell and form dystrophic growth cones. The alignment of the fibers allows the axons to grow outward along the alignment of the fibers. 79

Figure 3-7 Immunochemistry staining of 3-day DRG cultures on free-standing fiber scaffolds. The aligned, free-standing fiber scaffolds aided in axonal extension despite lack of underlying, supporting surface. 80

Figure 4-1: Synthesized PEDOT:PSS polymer after 24 hours of polymerization, double filtration and overnight drying. 93

Figure 4-2: Schematic of coaxial electrospinning. The inner and outer solutions are kept separate until they reach the nozzle. The two different nozzle designs that were 
tested are shown. A coaxial strand is emitted from the Taylor's cone at the tip of the nozzle and collected on a rotating, grounded collector to achieve aligned, coaxial fiber scaffolds

Figure 4-3: Different nozzle designs. (A) Stainless Steel nozzle (B) Teflon ${ }^{\circledR}$ nozzle (C) Dual needle nozzle

Figure 4-4: Coaxial Taylor's cone Left: During electrospinning, Right: Drawing. The inner solution can be seen as being fully encased in the outer solution. 101

Figure 4-5: FTIR spectrum of synthesized PEDOT:PSS. Major characteristic peaks are labeled. 104

Figure 4-6: Viscosity profile of PLLA solutions from viscosity testing. 105

Figure 4-7: Images obtained from fibers spun during viscosity testing. (A) $2 \mathrm{wt} \%$ solution (B) $4 \mathrm{wt} \%$ solution (C) $6 \mathrm{wt} \%$ solution (D) $8 \mathrm{wt} \%$ solution (E) $10 \mathrm{wt} \%$ solution . 108 Figure 4-8: Flow rate analysis for coaxial fibers. (A) $0.5 / 1$ (B) $1 / 1$ (C) $0.8 / 1.2$ (D) $0.5 / 1.5$ 109

Figure 4-9: Fluorescent optical images of core-sheath electrospun fibers. The upper left photo shows the red image taken, the upper left photo the green image taken and the bottom photo the overlay of the two. The consistent yellow fibers across the sample indicate successful formation of core-sheath fibers. 110

Figure 4-10: SEM image of core-sheath fiber comparing the lower detector image to the higher detector image. The brighter appearance of the sheath while the core has no change in appearance indicates the formation of core-sheath fibers with a conductive core and insulating sheath. 
Figure 4-11: SEM of smaller diameter core-sheath fibers. The sheath is split down the middle, revealing the conductive core inside. 113

Figure 4-12: Fibers spun with different diameters. The left-hand figure spun with22-17G system. Right hand spun with $22 \mathrm{G}-16 \mathrm{G}$ system. 114

Figure 4-13: IR spectrum for (upper) PLLA fibers and (lower) coaxial fibers. Key characteristic peaks are labeled. 116

Figure 5-1:Drawing and working prototype of stimulation cover. 129

Figure 5-2: Results of first round of chick DRG cell cultures on coaxial fibers. (A) 5-day DRG on pure PLLA (B) 7-day DRG on coaxial fibers with PEDOT nanoparticles (C) 7-day DRG on coaxial fibers with PEDOT:PSS (D) Glial stain of 5-2C (E) 5day DRG on coaxial fibers with PEDOT:PSS 132

Figure 5-3: Results from subsequent cell studies. Axonal outgrowth was not enhanced.

(A) 7-day culture on fibers with PEDOT:PSS core and large diameter (B) 5-day culture on fibers with a PEDOT:PSS core and small diameter (C) 5-day culture on fibers with a PEDOT:PSS core and large diameter (D) 5-day culture on fibers with oleic acid core 133

Figure 5-4: Results from electrical stimulation studies. (A) 24-hour stimulation and culture on stage, coaxial fibers with PEDOT:PSS core (B) Glial stain of A (C) 18hour stimulation and 5-day culture on PLLA, on coverslip (D) 18-hour stimulation and 5-day culture. 134

Figure 8-1: Left: A schematic of the electrospinning apparatus. (A) syringe pump, (B) syringe, (C) injector port, (D) needle with fiber strand production, (E) motor with speed control, (F) 6" aluminum wheel, $(\mathrm{G})$ vibration table, $(\mathrm{H})$ 
temperature/relative humidity meter, (I) humidity control with air flow, (J) light with high powered halogen bulbs, (K) camera, (L) TV monitor, (M) recorder. Right: Photo of spinning apparatus.

Figure 8-2: Video image of Taylor cone during spinning 171

Figure 8-3: 3D printed polylactic acid stages. The dimensions of the base are $1 \mathrm{~cm} x 1 \mathrm{~cm}$, height of the columns ranges from $3 \mathrm{~mm}$ to $4 \mathrm{~mm}$ in height. The stages are placed on the wheel and the fibers are spun across the columns so they are suspended above the base of the stage. 172

Figure 8-4: Optical microscopic images of the electrospun PLLA fibers. Spinning time was $23 \mathrm{~min}$ with a voltage of $17 \mathrm{KV}$ and a flow rate of $1.05 \mathrm{~mL} / \mathrm{hr}$. A and B show fibers spun on 3D stages with columns at $4 \mathrm{~mm}$ column height. $\mathrm{C}$ and $\mathrm{D}$ show fibers spun on flat coverslips. The samples were obtained on 12/11/14. 174

Figure 8-5: Fluorescent imaging of chick DRG cell cultures after 5 and 7 days. (A) shows a 5 day culture of a DRG performed on 3/19/15 seeded on fibers spun on a flat coverslip . The axons are growing straight outwards, along the alignment of the fibers. (B,C) 5 day cell culture performed on 3/19/15 and 7 day cell culture performed on 12/18/14. Both cells were seeded on stages. (B) The axons of the nerve cell seeded on the fibers spun on the stage still appear to be following the fibers, but it does not appear to have adhered very well. (C) The image is showing dual results. The right-hand side of the cell (I) appears to have axons following the fibers. However, the left-hand side of the cell and the top of the cell (II) appear to have grown vertically and jumped to another area of the fiber network. 
Fiber conditions for the samples cultured on 3/19/15 are 8 wet $\%$ solution spun for 23 minutes on $12 / 11 / 14$. 175

Figure 8-6: Yellow overlay created from red and green fluorescence images obtained from a coaxial fiber sample with PEDOT:DBSA nanoparticles in the core. Spun at $2500 \mathrm{rpm}$ wheel rotation speed and $16 \mathrm{G}$ outer needle. 181

Figure 8-7: Yellow overlay created from red and green fluorescent images from a coaxial fiber sample with PEDOT:PSS in the core. Spun at 2250rpm wheel rotation speed and $16 \mathrm{G}$ outer needle. 182

Figure 8-8: FE-SEM image obtained showing core-sheath structure. The core is pulled out of the sheath and is shown to be conductive. Apparant brightening of the sheath does not occur for the core fiber strand. 183 


\section{List of Tables}

Table 2-1: Key polymer solution parameters that effect electrospinning

Table 2-2: Key process parameters that effect electrospinning ................................... 39

Table 3-1 FFT Analysis of fiber alignment, fiber diameter and fiber density ................. 76

Table 3-2 Average axonal outgrowth for DRG culture study .................................... 81

Table 4-1: Viscosity data of PLLA solutions for viscosity profile.............................. 106

Table 4-2: Diameter, alignment and density data for different fiber samples ............... 114

Table 4-3: Mechanical testing data for coaxial fibers................................................. 117 


\section{Forward}

The work in this dissertation was done jointly with the research group of Dr. Feng Zhao in the Biomedical Engineering Department. Zichen Qian and Daniel Renke helped perform the various cell cultures that were used in the studies. Marie Wendling also aided in the cell cultures, as well as assisting with the spinning of fibers and the finalizing of the parameters. Daniel also assisted in writing the review article and Marie aided in article searching. Dr. Michael Mullins and Dr. Feng Zhao provided guidance in the writing of articles, the direction of the research and funding.

Dr. Joshua Pearce provided the 3D-printed scaffolds. Dr. Stephen Hackney performed the SEM imaging on the coaxial fibers. Dr. Ibrahim Miskioglu performed the nanoindentation testing on the coaxial fiber samples.

Chapter 2 will be submitted as a review article in Tissue Engineering Reviews B. Chapter 3 will be submitted as a research article in Journal of Neural Engineering. Chapter 4 will be submitted as a research article in Materials Science and Engineering C.

This work was presented at two national conferences. Some of the work in Chapter 2 was presented at the Materials Research Society Spring Meeting in San Francisco in April 2015. Some of the work in Chapters 2 and 3 was presented at the American Institute of Chemical Engineering National Meeting in San Francisco in November 2016. 


\section{Acknowledgements}

I would like to thank my advisor Dr. Michael Mullins for his guidance, insight and support during this project. I also want to thank Dr. Feng Zhao for agreeing to be my coadvisor and for her guidance throughout the project, specifically while Dr. Mullins was in Sweden on sabbatical. I would like to thank my committee members Drs. Caryn Heldt and Jeremy Goldman for agreeing to be on my committee and taking time out of their busy schedules to provide assistance.

Thank you to Dr. Joshua Pearce in the Materials Department at Michigan Technological University for suppling the 3D-printed stages for the elevated fiber scaffold work. Thank you to Dr. Stephen Hackney, also with the Materials Department, for assisting with the SEM imaging, doing much of the work pro bono. Thank you to Dr. Ibrahim Miskioglu with the Mechanical Engineering and Engineering Mechanics Department for assisting with performing the nanoindentation mechanical testing.

Thank you to all Marie Wendling, my undergraduate student researcher that has worked with me the past year and a half. She was a tremendous help in aiding me to reach various deadlines and motivating me to get work completed.

I would like to thank my family, especially my parents, for all their love and support throughout the years. I would not be where I am today without their guidance. I would like to specifically thank my mother for her encouragement throughout my collegiate career and for being such an extraordinary role model for me in all aspects of my life. I would not be who I am today without her.

Lastly, I would like to thank my wonderful and loving fiancé, Daniel Ring, for all his help and support. He was always there if I needed a shoulder to cry on or needed reminding of why I was working towards this goal. His patience while I have been in graduate school has been key to my successful completion. 


\begin{abstract}
A suitable tissue scaffold to support and assist in the repair of damaged tissues or cells is important for success in clinical trials and for injury recovery. Electrospinning can create a variety of polymer nanofibers and microfibers, and is being widely used to produce experimental tissue scaffolds for neural applications. This dissertation examines various approaches by which electrospinning is being used for neural tissue engineering applications for the repair of injuries to the central nervous system (CNS) and the peripheral nervous system (PNS). Due to the poor regeneration of neural tissues in the event of injury, tissue scaffolds are being used to promote the recovery and restoration of neural function. Next generation scaffolds using bioactive materials, conductive polymers, and coaxial fiber structures are now being developed to improve the recovery of motor functions in in vivo studies. This dissertation includes fabrication techniques, the results of neural cell cultures performed both in vivo and in vitro on electrospun fiber scaffolds, examines barriers to full functional recovery, and future directions for electrospinning and neural tissue engineering.
\end{abstract}

Aligned, free-standing fiber scaffolds using poly-L-lactic acid (PLLA) were developed as an in vitro model to study cell interaction on free-standing fiber scaffolds in vivo. Stages were designed to allow for the formation of free-standing fiber scaffolds that were not supported by an underlying surface. Fibers were spun across the columns of the stages to produce free-standing fiber scaffolds. The scaffolds were then used for in vitro cell culture using chick dorsal root ganglia (DRG). Fiber scaffolds were also spun on a flat substrate and used for in vitro cell studies for comparison. The axonal outgrowth 
observed for DRG cells cultured on free-standing fiber scaffolds was comparable to those grown on fibers with an underlying surface, indicating that cells follow the alignment of fibers even without an underlying support.

Electrospinning coaxial fibers is a more complex application of electrospinning techniques that has been explored here as a method of creating a core-sheath fiber structure to act as a scaffold across glial scar tissue present in spinal cord injuries (SCIs). Here, we looked at altering the basic electrospinning set-up to spin core-sheath fibers. The core was spun with a conductive polymer, poly(3,4-ethyelenedixoythiophene): poly(styrene sulfonate) (PEDOT:PSS) and the sheath was spun PLLA to create coaxial fibers with a conductive core and an insulating sheath. A conductive polymer was used so that electrical stimulation could be applied along the fibers during cell culture to examine if the additional external stimulation would further assist in axonal outgrowth when combined with the topographical cues of the fiber scaffolds. This allows for the combination of electrical stimulation with the topographical guidance provided by aligned fiber scaffolds to improve axonal outgrowth and functional recovery in vivo. 


\section{Introduction}

\subsection{Motivation and Organization of Dissertation}

Spinal cord injuries (SCI) and other neural tissue injuries to the central nervous system (CNS) and peripheral nervous system (PNS) continue to plague hundreds of thousands of people in the United States. Patients who experience injury to the PNS often experience site numbness, neural tissue death, and lack of function or disability. Patients who experiences SCI and injury to the CNS often experience various forms of paralysis, loss of function, life-long disability and even death. These injuries cost patients millions of dollars each year. The complex nature of neural tissue makes self-regeneration extremely limited, so these injuries do not heal on their own and require some sort of clinical intervention. There is currently no treatment available that allows for full functional recovery after SCI. Recently, neural tissue scaffolds made from electrospun polymer fibers have shown promise in acting as a bridge over scar tissue present after SCI and allow for axonal reconnection. Aligned polymer scaffolds produced from electrospinning have shown to aid in axonal outgrowth in vitro and have shown in vivo to regenerate spinal cord tissue and aid in recovery to peripheral nerve tissue. However, there is still no clinically approved method for which to use the fiber scaffolds as treatment for these injuries. 
Electrospinning is a technique that has been extensively studied within the past decade and a half as a method to produce scaffolds for a variety of tissue engineering applications. The benefits of using electrospun fiber scaffolds for tissue engineering is that the scaffolds can be made to mimic the natural extracellular matrix (ECM) and allow for the transfer of proteins, water, and other nutrients into the tissue. The electrospun fiber mats can be used as a scaffold to support new tissue growth. Cardiovascular, bone, skin, and neural tissues have all been explored as possible applications for electrospun tissue scaffolds to repair damaged or diseased tissue. Each type of tissue has different requirements as far as mechanical properties, degradation rates of implants, healing time, and pharmaceuticals such as growth factors. The tunable nature of the electrospinning technique makes it a very attractive method for producing tissue scaffolds for repair of multiple types of tissues, as it allows for the use of different polymers and parameters to achieve the desired resulting scaffold with the required properties. Electrospinning has also been used to create drug delivery scaffolds and has been used in dialysis treatments as an alternative to machines to remove toxins from individuals experiencing kidney failure.

In this dissertation, we aim to further the understanding of the interactions of axons with the fiber scaffolds in vivo. We also aim to develop more complex fiber structures that will improve the efficacy of the scaffolds and further aid axonal outgrowth, increasing the possibility of clinical use for repair of SCI and other neural injuries. Electrospinning presents many variables and challenges that need to be considered to successfully spin consistent fibers for scaffolds. Despite these challenges, 
the technique offers a great deal of flexibility to spin fibers made of different polymers, fibers with different diameters, and fibers with complex structures, such as core-sheath structures, and is a way to create fiber scaffolds directly. The option to use a variety of polymers and techniques with electrospinning allows for the opportunity to use nerve growth factor (NGF), other biocompatible pharmaceutical agents, conductive polymers, proteins, etc. in the electrospinning process. Having fibers that contain some of these other agents can help improve the biocompatibility and restorative properties of the scaffolds. Spinning fibers with these agents can be done either by creating blends with the base polymer solution or in core-sheath fibers where the desired component, such as NGF, is imbedded in the core of a core-sheath fiber structure with a polymeric sheath. Imbedding NGF and other biochemical agents in the core helps limit the burst release often associated with in vivo delivery of pharmaceuticals and keeps it viable in the body for a longer period, making the growth factor more useful and available throughout the healing process.

Using conductive polymers in the formation of electrospun fiber scaffolds may assist in the repair of neural tissue. Neural tissue is electrical in nature due to the concentration of salt ions present in the tissue. It has been documented that axonal outgrowth is affected by electrical stimulation and in some studies a degree of function was restored to PNS injuries when the tissue was exposed to electrical stimulation during regeneration. However, electrical stimulation has not provided a method for full tissue and functional regeneration. It is our hypothesis that by using the external cues of electrical stimulation with the topographical cues provided by aligned polymer fiber 
tissue scaffolds in a combined strategy approach, the regeneration of tissue and restoration of function can be increased and eventually used in a clinical approach.

This dissertation is split into four main chapters, each with their own aims and objectives, with a final conclusions and future works chapter. The aim of Chapter 2 is to explore the science behind electrospinning and the various parameters. This is key in moving forward for designing experiments in which to electrospin various fiber scaffolds. It also explores the biology behind the nervous system, and the obstacles that present when injury occurs. We also sought to explore the research that has been done in studying the use of electrospun scaffolds for tissue engineering applications, with an emphasis on neural tissue, through a literature review. This also allowed us to search for opportunities and gaps in the research. We could then design our approach based on what had been previously studied and what had yet to be explored. While other comprehensive electrospinning reviews have been written, ours is unique in that it explores the electrospinning technique itself in detail, focuses on the neural tissue application of electrospun fiber scaffolds, and explores complex fiber structures in one review.

Chapter 3 explores the spinning of poly-L-lactic acid (PLLA) fiber scaffolds across the columns of an elevated stage to create scaffolds with fibers suspended over the surface. This work was performed in response to criticism by reviewers of the previous research about the growth mechanism of axons in vitro on the fiber scaffolds. Suspending the fibers over a surface and then performing in vitro tissue cultures on the scaffolds removed the two-dimensional constraint of performing the cultures on a flat surface. The aim of the work was to determine whether the axonal outgrowth observed was due to the 
topographical cues provided by the aligned fiber scaffold or was because of the constraint of the two-dimensional system. The previous work was performed on flat coverslips and it was the opinion of some reviewers that the extensive axonal outgrowth observed was caused by the axons following the channels between the fibers or along the surface of the coverslip rather than due to the aligned fiber scaffolds. It was our hypothesis that the fibers were providing the topographical cues for axonal outgrowth and suspending the fibers would show results similar to those seen previously.

To study this criticism, an elevated stage was designed that would allow fibers to be directly spun across the columns of the elevated stage and thus be suspended above the surface of the stage. Cell tissue cultures using chick dorsal root ganglia (DRG) similar to those performed previously were then performed to determine the growth mechanism. The design of an elevated stage was carefully considered and studied, and eventually produced using 3D-printing and classic machining methods. By producing elevated fiber scaffolds that would create a three-dimensional tissue environment, the interactions of the axons with free-standing fiber scaffolds in an in vivo environment when DRG cells were implanted on the scaffolds could also be studied and modeled. Analysis of the fiber diameter, alignment and density of different fiber scaffolds that were used in cell culture studies was also performed. This was done to compare the free-standing fiber scaffolds to the fibers spun on a flat surface. The results are summarized in Chapter 3 and show that our hypothesis was correct. This work was unique in that is the first time that suspended fibers were spun directly onto a free-standing support that was not connected directly to 
ground. Partial completion of this work was published as conference proceedings for the Annual Spring 2015 Materials Research Society Meeting and can be seen in Appendix A.

Chapter 4 was performed to examine the potential of using electrospun coaxial fibers with a conductive core and insulating sheath as fiber scaffolds for repair of neural tissue. Much work was done to examine and finalize the parameters to spin consistent coaxial fibers. Different coaxial nozzles that had been previously designed were tested to determine the proper nozzle to use to spin consistent coaxial fibers. Fibers were spun with different spinneret combinations to determine the effect on fiber parameters and cell culture. Different core polymer solutions were also tested to determine the correct core polymer to use to produce coaxial fibers. A conductive polymer was then polymerized through oxidative polymerization and then imbedded in the core solution of the coaxial fibers so fibers could be spun with a conductive core and an insulating PLLA sheath. The idea was to create a type of artificial axon and then examine axonal interaction and outgrowth with the new fibers. The specific methods used are outlined in Chapter 4. Coaxial fibers with a conductive core were successfully spun as determined through various analytical techniques such as FTIR and optical imaging. Fiber properties such as diameter, alignment, density, modulus and stiffness were determined through analytical techniques using imaging software and nanoindentation. While coaxial fibers and conductive polymers have been studied for use in neural tissue scaffolds, this work distinguishes itself by the conductive polymer used as the conductive core, the imaging techniques and the creation of an "artificial axon". 
Fibers spun both on flat coverslips and stages were used for examination. To study the axonal interactions, in vitro chick DRG cultures were performed. The effect of electrical stimulation on axonal outgrowth was also studied. A stimulation chamber was designed using the cover of a six-well culture plate and carbon electrodes that would supply voltage either through solution medium for fibers on a flat coverslip or across fibers when placed on either side of a three-dimensional stage. The results are summarized in Chapter 5.

The final chapter lists the overall conclusions of each chapter and the recommendations for future work to further the research. The suspended scaffold work showed that the axons follow the alignment of fibers during outgrowth and show that this is the behavior experienced in vivo when the scaffolds are implanted as a bridge. The coaxial work showed that conductive, coaxial fibers with a conductive core and insulating sheath were produced. The idea of using conductive coaxial fibers is to eventually communicate with individual axons during regeneration and effect the axonal outgrowth and reconnections to achieve successful regeneration of neural and spinal cord tissue and restore motor function of those who have suffered SCI.

\subsection{Objectives}

Electrospinning scaffolds for neural tissue applications has shown much promise. This project aims to build on previous work done by other researchers to increase the efficacy of neural tissue scaffolds. I hypothesize that by manipulating the electrospinning process, spinning fibers on a new platform, and spinning novel coaxial structures, a scaffold can be produced that will act as a growth permissive bridge across the glial scar 
and can be used in spinal cord injury (SCI) repair. There are two specific aims that are addressed in this dissertation, each with various tasks that were performed in order to achieve the aims.

Specific Aim 1: Spin free-standing fiber scaffolds without a supporting, underlying surface and to determine the effect of topographical cues provided by the fibers on axonal outgrowth.

Task 1: Design and construct an elevated stage to spin free-standing fibers and perform electrospinning using stages.

Task 2: Perform in vitro DRG axonal outgrowth studies on the elevated scaffolds.

Specific Aim 2: Use a modified electrospinning nozzle to spin coaxial fiber scaffolds, both on flat coverslips and elevated stages, with a conductive core and insulating sheath.

Task 1: Determine electrospinning parameters such as different flow rates, spinning wheel speeds, voltage value, etc. to spin consistent coaxial fibers.

Task 2: Synthesize conductive polymer PEDOT:PSS through oxidative polymerization methods and use characterization methods to determine proper synthesis. Preform electrospinning using synthesized polymer in the core.

Task 3: Use imaging techniques such as Fluorescence Optical Microscopy and Scanning Electron Microscopy to determine presence of coaxial fibers.

Task 4: Preform in vitro cell cultures on coaxial fibers to determine efficacy of fiber scaffolds and effect on axonal outgrowth. 
Task 5: Perform preliminary studies in vitro DRG studies with electrical stimulation along the fibers. 


\section{Electrospinning Techniques for Use in Neural Tissue}

\section{Engineering ${ }^{1}$}

\subsection{Abstract}

A suitable tissue scaffold to support and assist in the repair of damaged tissues or cells is important for success in clinical trials and for injury recovery. Electrospinning can create a variety of polymer nanofibers and microfibers, and is being widely used to produce experimental tissue scaffolds for neural applications. This chapter examines various approaches by which electrospinning is being used for neural tissue engineering applications for the repair of injuries to the central nervous system (CNS) and the peripheral nervous system (PNS). Due to the poor regeneration of neural tissues in the event of injury, tissue scaffolds are being used to promote the recovery and restoration of neural function. Scaffolds produced from polymers such as poly-lactic acid (PLA), poly(caprolactone) (PCL), and poly(L-lactide-co-glycolide) acid (PLGA) have shown promise in facilitating nerve regrowth and axonal guidance over the past decade. Next generation scaffolds using bioactive materials, conductive polymers, and coaxial fiber structures are now being developed to improve the recovery of motor functions in in vivo studies. This chapter includes fabrication techniques, the results of neural cell cultures performed both in vivo and in vitro on electrospun fiber scaffolds, examines barriers to

\footnotetext{
${ }^{1}$ The material within this chapter has been submitted for review to the journal "Tissue Engineering $B$ Reviews."

Citation:

Martin, Rachel, Wendling, Marie, Radke, Dan, Zhao, Feng and Mullins, Michael. "Electrospinning Techniques for use in Neural Tissue Engineering Applications."
} 
full functional recovery, and future directions for electrospinning and neural tissue engineering.

\subsection{Introduction and Motivation}

One of the most significant areas in which electrospun fiber scaffolds are being applied is in repairing neural tissue. Neural tissue is the main component of the both the central nervous system (CNS) and the peripheral nervous system (PNS). Neural tissue is made up of two different types of cells, neurons and neuroganglia ${ }^{1}$. Neurons connect the entire body to the brain and spinal cord, while the neuroganglia support and protect the neurons ${ }^{1}$. The neuron is made up of a cell body, dendrites and an axon. Dendrites are nerve fibers that extend from the cell body and receive or input information from different parts of the body. Axons relay nerve impulses until the impulses reach their destination ${ }^{1}$. Damage to the nervous system results in crushed or severed axons, causing a disruption in neural impulses. Due to a lack of regenerative capabilities of neural tissues, these injuries are debilitating and costly, resulting in decreased life expectancy and fatalities. As of 2016, the rate of spinal cord injury (SCI) in the United States was about 17,000 new cases per year ${ }^{2}$. There are estimated to be around 282,000 people currently living with some degree of SCI in the United States ${ }^{2}$. In incidents of SCI, over $40 \%$ of cases are classified as being complete quadriplegia, and $0.4 \%$ experience complete recovery after any degree of $\mathrm{SCI}^{2}$. The cost of injury to someone who has experienced quadriplegia is over $\$ 1,000,000$ in the first year and over $\$ 180,000$ for each subsequent year. Even those who experience some recovery of motor function still experience a cost of almost $\$ 350,000$ for the first year and over $\$ 40,000$ for each subsequent year ${ }^{2}$. The life 
expectancy of someone with a quadriplegic injury at the age of 20 is only 55, and the life expectancy drastically decreases as the age at the time of injury increases ${ }^{2}$. As a result of the high cost of these injuries, the frequency of occurrence and poor prognosis, new medical interventions are necessary to provide an opportunity for repair of neural injuries.

\subsection{Neural Tissue Structure and Injury}

The CNS and PNS are made up of different types of neuroganglia, with the CNS possessing 4 types and the PNS possessing 2 types ${ }^{1}$. The CNS contains three types of glial cells, consisting of two types of macroglial cells, astrocytes and oligodendrocytes, and one set of microglial cells ${ }^{3}$. The CNS also contains ependymal cells, which produce cerebrospinal fluid. Cerebrospinal fluid protects the brain and spinal cord in the CNS ${ }^{1}$. Microglial cells act as macrophages that are activated by injury, disease, infection and cell death. The two types of neuroganglia that reside in the PNS are Schwann cells and satellite cells. Satellite cells are flat cells that provide structural support by surrounding the cell bodies and regulate the transfer of materials within the PNS. Schwann cells, which myelinate the axons in the PNS, are surrounded by neurolemma, which aid in regeneration and are not present in the $\mathrm{CNS}^{1}$.

Injuries to both the PNS and CNS are often traumatic, due to lack of regeneration and neurogenesis. When the spinal cord in the CNS is severed or crushed upon injury, the cell body can be damaged, which causes functional loss 3 . There are a variety of growth inhibitory cues the neural tissue experiences after injury which prevents regeneration: (1) myelin debris can accumulate and act as a barrier to regeneration, (2) astrocytes and 
oligodendrocytes can also be overstimulated in response to injury, (3) the glial cells over time cause the formation of a glial scar, which acts as a barrier to regeneration and axonal extension, and (4) the expression of proteoglycans are also stimulated by the formation of the glial scar tissue, and halt the regeneration of CNS tissue ${ }^{3}$ (Figure 2-1). While both inhibiting and promoting molecules are up-regulated in the response to injury to the CNS tissue, the inhibitory cues experience a higher level of up-regulation ${ }^{4}$. Because there is some up-regulation of promoting molecules, the severed axons do experience some extension at their ends, but become deformed in the presence of inhibitory molecules and form misshapen, dystrophic growth cones within the scar tissue. This causes the axons to stop growing and regenerating, and they become entangled with other axons during regrowth ${ }^{4}$. Due to the combination of the inhibiting growth molecules and the lack of Schwann cells and neurolemma, the CNS does not experience any significant selfregeneration.

The PNS has some ability for self-repair, but only if the cell bodies remain intact and if Schwann cells are present and active ${ }^{1}$. However, neural regeneration can only occur over very small gaps. For a gap of $2 \mathrm{~cm}$ or less, recovery is possible, but can be moderate and full functional recovery may not occur. With gaps of $2 \mathrm{~cm}$ or larger, selfrecovery is not possible and requires surgical intervention ${ }^{5}$. In some injuries to the PNS, the severing of the nerve occurs, causing so much damage that regeneration cannot occur, and surgery is required ${ }^{6}$. Even in instances where surgery is required, there is a strict time-line after which surgery is no longer effective. Within 12-18 months of the injury 
having occurred, permanent atrophy sets in due to the lack of movement caused by the nerve injury, thus rendering surgery ineffective ${ }^{6}$.

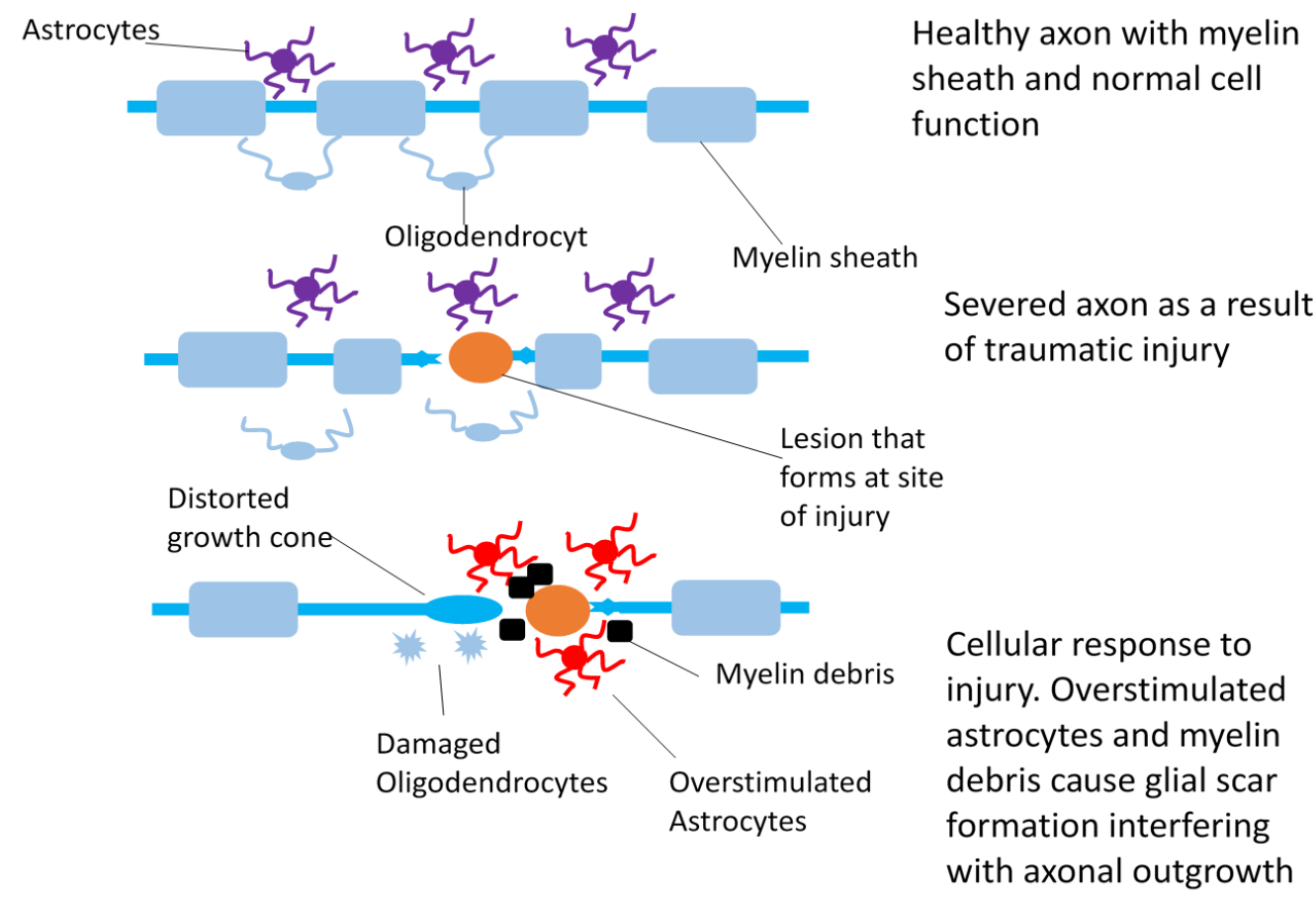

Figure 2-1: Cellular response to injury to the CNS. 
There are clinical methods currently used to surgically repair injuries to the PNS when the gap in the injured nerve is greater than $2 \mathrm{~cm}$. Nerve autografts produce the best results, but they also present risk of complications after implantation. They often leave patients with donor site morbidity and significant disability even under optimal circumstances ${ }^{7}$. Another method for repairing damaged nerves is coaptation, which draws together the two nerve ends to repair the nerve. This can be done with two nerves side by side, two nerves end to end, two nerves side to end, or with an autograft ${ }^{8}$. Coaptation can only be used for nerve injuries with small gaps, with anything more than $20 \mathrm{~mm}$ requiring a different type of treatment. There is still a risk of site morbidity and residual nerve damage ${ }^{8}$.

In the event of a spinal cord injury (SCI), it may be necessary to bridge a significant gap and the glial scar. In both cases, a scaffold may be used to facilitate and provide guidance for axonal outgrowth. Much work has been focused on the creation of a natural or polymeric scaffold with the current method being the use of autografts or allografts ${ }^{9-11}$. Autografts are nerve grafts in which neural tissue is taken from a different part of the body of the injured individual and grafted into the site of injury in injuries to the PNS. In an allograft nerve graft, neural tissue is taken from a donor individual and then grafted into the site of injury. Autografts are only used in the case of PNS injury and have numerous drawbacks. Allografts often result in rejection or infection, and in the case of both allografts and autografts, there is a shortage of available tissue ${ }^{7}$. More effective clinical solutions are needed to repair both PNS and CNS injuries. Synthetic alternatives to nerve grafts are being explored, which include hydrogels ${ }^{12}$, nerve growth 
factors ${ }^{13}$, and implantable scaffolds as growth conduits. Current neural scaffolds focus primarily on providing physical guidance cues to developing axons through the use of channels or conduits fabricated from polymers or biologically derived materials, often placing emphasis on the surface morphology to promote alignment ${ }^{14-17}$. Conduits should be mechanically pliable and highly aligned to mimic the structure of natural neural tissue while having enough structural strength to support the regeneration SCs, neurons, and connective tissues, and would ideally be biodegradable and utilize biochemical growth signaling to replace the graft with native tissues over time without the loss of function. Hydrogels have been reported to support high cell viability and stem cell differentiation $^{12}$, and support the formation of a functional neural cellular network ${ }^{18}$. Highly aligned fibrillary fibrin (AFG) hydrogel has been used to mimic the soft and aligned structure of natural nerve tissue. Yao et al. found the physical cues caused dorsal root ganglion (DRG) neurons to rapidly grow aligned to the AFG fibers without being supplemented by neurotropic factors ${ }^{19}$. This suggests physical guidance may dominate the regeneration process of peripheral neurons, and that scaffolds need only recreate the proper surface interactions and bulk mechanical properties of the natural connective tissue sheath for functional repair. In particular, the use of electrospinning techniques to form polymeric fiber scaffolds to bridge nerve gaps of $2 \mathrm{~mm}$ or larger and promote growth across glial scars has shown promise ${ }^{20}$. Fiber scaffolds provide surface cures for the axons during outgrowth. Without such guidance cues, the axons extend out from the cell body and become entangled, creating a halo-like structure around the cell body called dystrophic growth cones. By providing an aligned fiber surface for axonal outgrowth, the outgrowth can be controlled and directed. Figure 2-4A shows a chick dorsal root ganglia 
(DRG) cell that has been cultured without underlying growth cues. The axonal outgrowth is entangled around the cell body and dystrophic growth cones are present.

\subsection{Electrospinning}

Electrospinning is a technique used to create polymeric fibers that are of the nanoscale, as small as tens of nanometers, or of the microscale, as large as several microns $^{21}$ (Figure 2-2). Electrospinning provides a direct way to spin polymeric fibers ${ }^{21}$. There are three basic pieces of equipment required for small scale electrospinning, including a spinneret, being either a nozzle or metallic needle, a high voltage power supply, and a grounded collector ${ }^{22}$. Electrospinning can be done under either a positive or negative electric field, and the collector can be grounded or of the opposite charge to the electric field. A viscous polymer solution is pumped to the tip of the spinneret that is connected to a high voltage power supply. If the potential at the tip of the spinneret is sufficient to overcome the surface tension of the solution droplet formed at the tip of the spinneret, the electrostatic forces will distort the droplet. When an equilibrium between the two forces is reached, it causes the formation of what is known as a Taylor's cone ${ }^{21}$. Once the electrostatic forces become dominant, the cone is further distorted to the point that one or more polymeric strands are emitted from the tip of the cone. The charged strands are then attracted to a grounded collector to maintain electroneutrality (Figure 22).

Both the polymer solution properties and the electrospinning process parameters need to be considered for electrospinning fibers. Much work has been done to examine and mathematically model these parameters and their effects on electrospinning ${ }^{21,23,24}$. 
However, due to the large number of electrospinning variables, electrospinning remains almost as much of an art as a science. There are several parameters that have a significant effect on the electrospinning process, and we will highlight a few.

Relevant solution properties include surface tension of the droplet, density of the solution, viscosity of the solution and the charge density. A list of the important solution properties is listed in Table 2-1. The polymer should be of sufficient molecular weight to be electrospun, and this is determined by the degree of chain entanglements that occur when the polymer is in solution ${ }^{25}$. The typical molecular weight of polymers used in electrospinning is between $30 \mathrm{kDa}$ and $400 \mathrm{kDa}^{22}$. The polymer solution must contain charge carriers to interact with the electric field in order for the electrospinning process to occur $^{22}$. The charge carriers can be provided by the solvent or by the solution itself. Charge carriers provide a charge density in the solution and allow the transfer of an electric charge from the power supply to the solution at the tip of the spinneret. Charges build up on the surface of the Taylor's cone and travel to the grounded or oppositely charged collector.

The concentration and viscosity of the polymer solution are important factors in electrospinning. The minimum viscosity that is required is a value at which the concentration of the solution is greater than the critical concentration. The critical concentration is determined by the average molecular weight of a polymer and the solvent, so it varies with each polymer solution ${ }^{25}$. Typical solvents used in electrospinning include acetone, chloroform, dichloromethane and toluene ${ }^{25}$. The nozzle feed rate also has an impact on the size and diameter of the fibers that are collected. It is 
important to have a balanced flow, with the feed rate of the polymer solution matching the rate at which the polymer strand is being pulled from the tip of the spinneret ${ }^{25}$. Typical feed rates generally range from $0.5 \mathrm{~mL} / \mathrm{hr}$ to $4 \mathrm{~mL} / \mathrm{hr}$.

Table 2-2 shows a list of process parameters which apply to the electrospinning environment and equipment used that effect the formation and morphology of fibers. These include applied voltage, collector geometry, gap distance between the spinneret and collector, electric field strength, spinneret diameter and design, and the electrospinning environment. The grounded collector used can have a great impact on the geometry of the fiber mats collected. Using a stationary plate or foil is the most common and creates a random, disordered two-dimensional mat of fibers ${ }^{7,25-31}$ (Figure 3-3). If the collector is moving or rotating, the density of the mat formed can be controlled, allowing for some control of the geometry of the mat. The formation of aligned or ordered fiber

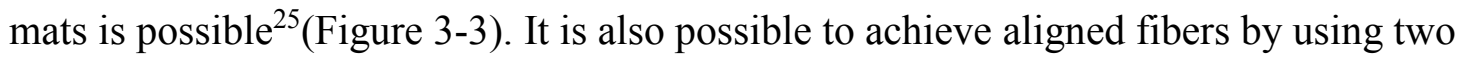
charged plates placed at a certain distance apart from each other. The plates are of the opposite charge as the charged fiber strand emitted from the Taylor's cone, or are grounded. As the fiber strand whips, it becomes attracted to the closer plate and then is whipped to the adjacent plate. As the charged strand whips back and forth between the two plates, and aligned fiber mat is formed ${ }^{25}$.

The applied voltage effects the morphology of the fibers. A higher voltage results in greater elongation of the jet and a greater instability, which results in a smaller diameter fiber ${ }^{25}$. The gap distance between the spinneret and the collector effects the applied electric field during electrospinning, as the applied electric field is directly related 
to the ratio of the applied voltage to the gap distance. Shortening the gap distance without changing the applied voltage will increase the electric field. If the gap distance is not large enough, there will not be enough time for the solvent to evaporate and the fibers to solidify. If the gap distance is too large, the field strength will not be high enough to overcome the surface tension of the droplet. Although the ideal voltage field is dependent on the electrical and physical properties of the electrospinning solution, the range is typically between 1 and $2 \mathrm{kV} / \mathrm{cm}$. The humidity and temperature of the environment effects the electric field and drying time of the fibers, and must be properly controlled and in balance in order to spin uniform fiber mats ${ }^{32}$.

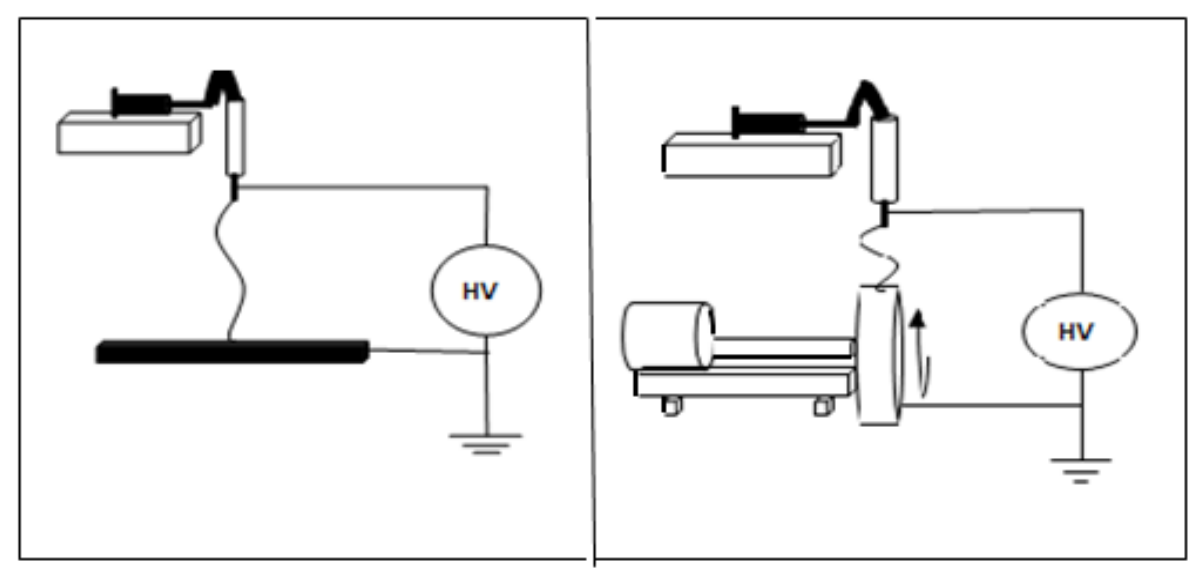

Figure 2-2: Electrospinning apparatus for collection on a flat, stationary collector and a rotating wheel collector 
Table 2-1: Key polymer solution parameters that effect electrospinning

\begin{tabular}{|c|c|c|}
\hline $\begin{array}{c}\text { Solution } \\
\text { Parameter }\end{array}$ & Symbol & Parameter function variables \\
\hline $\begin{array}{l}\text { Flow rate } \\
(\mathrm{mL} / \mathrm{hr})\end{array}$ & $Q$ & viscosity, concentration \\
\hline $\begin{array}{l}\text { Solution } \\
\text { density } \\
(\mathrm{g} / \mathrm{mL})\end{array}$ & $\rho$ & solvent, polymer, concentration \\
\hline $\begin{array}{l}\text { Solution } \\
\text { viscosity } \\
\text { (cP) }\end{array}$ & $\boldsymbol{\mu}$ & solvent, polymer, concentration \\
\hline $\begin{array}{l}\text { Solution } \\
\text { concentration } \\
\text { (wt.\%) }\end{array}$ & $c$ & solvent, polymer \\
\hline $\begin{array}{l}\text { Polymer type } \\
\text { and length } \\
(\mathrm{kDa})\end{array}$ & $M_{w}$ & polymer, fiber application, dielectric constant \\
\hline Solvent type & - & polymer \\
\hline $\begin{array}{l}\text { Vapor } \\
\text { pressure } \\
(\mathrm{kPa}) \\
\end{array}$ & V.P. & solvent \\
\hline $\begin{array}{l}\text { Surface } \\
\text { tension } \\
(\mathrm{N} / \mathrm{m}) \\
\end{array}$ & $\sigma$ & polymer, viscosity, solvent, concentration \\
\hline $\begin{array}{l}\text { Dielectric } \\
\text { constant }\end{array}$ & $\boldsymbol{k}$ & polymer, solvent \\
\hline
\end{tabular}


Table 2-2: Key process parameters that effect electrospinning

\begin{tabular}{|l|c|c|}
\hline \multicolumn{1}{|c|}{$\begin{array}{c}\text { Process } \\
\text { Parameter }\end{array}$} & Symbol & Parameter function variables \\
\hline $\begin{array}{c}\text { Applied voltage } \\
(\mathrm{KV})\end{array}$ & $\boldsymbol{V}$ & $\begin{array}{c}\text { polymer type, concentration, viscosity, solution } \\
\text { conductivity, solution surface tension }\end{array}$ \\
\hline $\begin{array}{l}\text { Electric field } \\
\text { strength(KV/cm) }\end{array}$ & $\boldsymbol{E}$ & applied voltage, electrode spacing \\
\hline $\begin{array}{c}\text { Gap distance } \\
(\mathrm{cm})\end{array}$ & $\boldsymbol{L}$ & solvent, solution conductivity \\
\hline $\begin{array}{l}\text { Spinneret } \\
\text { diameter and } \\
\text { design (mm) }\end{array}$ & $\boldsymbol{d}$ & polymer, viscosity, fiber application \\
\hline $\begin{array}{l}\text { Temperature } \\
\left({ }^{\circ} \mathrm{C}\right)\end{array}$ & $\boldsymbol{T}$ & solvent, polymer, density \\
\hline Humidity $(\%)$ & $\boldsymbol{R} \boldsymbol{H}$ & fiber application, electric field polarity, fiber \\
& conductivity \\
\hline $\begin{array}{l}\text { Collector } \\
\text { material and } \\
\text { geometry }\end{array}$ & - & \multicolumn{2}{|l}{} \\
\hline
\end{tabular}

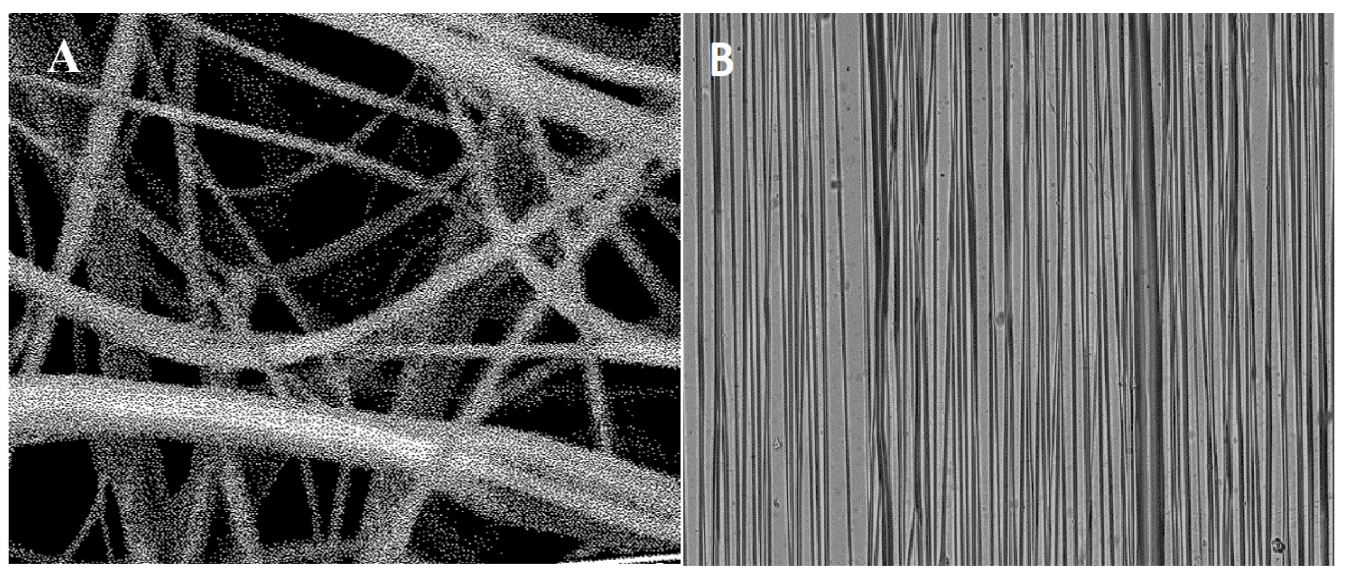

Figure 2-3: Poly-L-lactic acid nanofibers (A) random fibers on a station collector (B) aligned fibers on a rotating collector

Electrospinning techniques use many different polymers and a variety of both natural and synthetic polymers have been explored as neural tissue scaffolds. Polylacitc 
acid (PLA) $)^{26,27,33-37}$, silk fibroin (SF) $)^{38}$, poly(caprolactone) (PCL) $)^{15,28,39,40}$, poly(L-lactideco-glycolide) acid (PLGA $)^{29,30,41}$, cellulose acetate butyrate ${ }^{40}$ as well as composite

polymeric solutions ${ }^{7,31,42-45}$ have been used to produce fiber scaffolds for neural tissue. A grounded rotate collector can create aligned fiber mats. By changing the arrangement of the fibers within the mat it is possible to alter the interactions between the cells and the fiber scaffold. Aligned fiber scaffolds have shown more promise in guiding axonal outgrowth during in vitro cell cultures than randomly aligned fiber mats ${ }^{33-35}$.

\subsection{Applications of Electrospun Fibers}

The technique of electrospinning has attracted a great deal of attention within the last two decades as a useful and direct way to produce fibers that can be used as scaffolds in tissue engineering applications ${ }^{9,22}$. In tissue engineering, there are three main components that all play vital roles: (a) cells, (b)tissue scaffolds and (c) signaling molecules ${ }^{46}$. Different cell types, such as cardiac cells or neural cells, may require different materials or different approaches. For example, cardiac tissue, which experiences a great deal of mechanical stress, requires the use of materials that can withstand the amount of stress in the cardiac environment. Neural tissue, being highly ordered in structure, requires materials that can mimic the natural environment. Signaling molecules can be used to enhance the growth of implanted tissues and are usually cellspecific. Tissue scaffolds are used to provide support for existing or implanted cells and tissue and are sometimes used to deliver signaling molecules. While all three elements are equally important, it is the scaffolds to which electrospinning methods are applicable. Scaffolds act as a platform to support cells during the regeneration process and if stem 
cells are used, allow cells to spread and differentiate into the desired tissue ${ }^{46}$. Scaffolds also allow for the entry of various nutrients, proteins, water, growth factors and other vital biomaterials to enter the cell system during regeneration, helping to result in the formation of healthy tissues.

\subsubsection{Fiber scaffolds in tissue engineering}

Fibers obtained using electrospinning methods have been used in bone, neural, cardiovascular, and cartilage tissue engineering using various polymers and electrospinning techniques. Injury and degenerative diseases dictate the need for bone repair methods such as implants, transplants, and other techniques ${ }^{47}$. The current repair methods have various restrictions such as infection, nerve injury, and the risk for disease transfer ${ }^{47}$. These limitations necessitate the development of new technology to repair various chondral defects and injuries. Nanofibers produced by electrospinning, given their high surface area to volume ratio, flexibility for a wide range of polymers, and continuous structure give these scaffolds the mechanical properties necessary in order to mimic natural bone tissue ${ }^{47}$. The fibrous nature of the scaffolds mimic the fibrous nature of bone tissue ${ }^{48}$. Xu et al. showed that electrospun nanofibers can be altered in such a way as to promote a higher potency chrondogenic differentiation in mouse bone marrow mesenchymal stem cells, showing the vast applications of this technique and its potentials in tissue engineering ${ }^{49}$.

Cardiovascular disease continues to be the leading cause of death worldwide ${ }^{50}$. Heart attacks are among the leading causes of deaths related to cardiovascular diseases. Cardiac tissue has a very limited capacity for regeneration after an instance of a heart 
attack, leading to a lack of ability to restore normal function to the heart ${ }^{51}$. Heart transplants continue to be the best way to regenerate cardiac function, but are extremely limited due to the lack of donors and possibility for rejection or other serious and lifethreatening side-effects ${ }^{51}$. Electrospinning as a tissue engineering application is emerging as a promising alternative approach to regenerate existing cardiac tissue using an implanted scaffold.

\subsubsection{Aligned fiber scaffolds for neural tissue repair}

Given the highly-structured nature of neural tissue, researchers have found that spinning aligned fiber scaffolds have improved the function of the scaffolds for neural tissue repair ${ }^{34,35}$. Aligned scaffolds have shown an increase in axonal extension during both in vitro and in vivo cell cultures (Figure 2-4). One of the most studied polymers is poly-L-lactic acid(PLLA). PLLA has proven to be an effective polymer in numerous cases in in vitro studies for neural applications. PLLA seems to allow just enough adhesion to prevent the neural cells from falling off the scaffolds but does not have such high adhesion that it hinders axonal outgrowth. It is also biocompatible and biodegradable. By electrospinning aligned PLLA nanofibers, Corey et al. were able to show that highly aligned fiber mats increased the neurite outgrowth of the dorsal root ganglia (DRG) of Sprague-Dawley rat embryos cultured in vitro $20 \%$ over random fiber mats $^{33}$ (Figure 2-5). It was shown that the neurites extended parallel to the direction of the fibers, and on aligned fibers extended $760 \pm 71 \mu \mathrm{m}$. In a similar study, Wang et al. demonstrated that highly aligned fiber mats produced from specialized electrospinning parameters showed extensive neurite outgrowth of stage E9 chick DRG cultured in vitro, 
and also demonstrated that the occurrence of crossing fibers in the scaffold that fall out of alignment inhibit neurite outgrowth, in some instances stopping outgrowth altogether when the neurite comes into contact with the junction of crossed fibers ${ }^{34}$ (Figure 2-6). The highly aligned mats produced by Wang showed that $84 \%$ of fibers deviated by only $\pm 2^{\circ}$ from a parallel lined as measured using Fast Fourier Transform analysis, and 99\% of fibers deviated by $\pm 10^{\circ 34}$. Neurite extension on the studies performed by Wang was measured at $1306 \pm 204 \mu \mathrm{m}$ on aligned fiber mats as opposed to $854 \pm 204 \mu \mathrm{m}$ when cells were cultured on a flat PLLA film, indicating an increase of neurite outgrowth of $53 \%{ }^{3}$. They also showed that an increase in fiber density within the mat resulted in an increased number of extended neurites. In a later study, the same group demonstrated that altering the diameter of the fiber can have a positive impact on the outgrowth of neurites and on the migration of Schwann cells (SCs), an important factor in PNS repair. Schwann cells are cells that are present in the PNS and assist in the regeneration process. Wang demonstrated that fibers with a larger dimeter of $1325 \pm 383 \mathrm{~nm}$ exhibited longer neurite outgrowth than those with the smallest diameter of $293 \pm 65 \mathrm{~nm}$, indicating that fibers in the micrometer range are better suited to aid in neural tissue repair. The fibers with the largest diameters exhibited the greatest packing density, resulting in the greatest SC migration $^{35}$. 


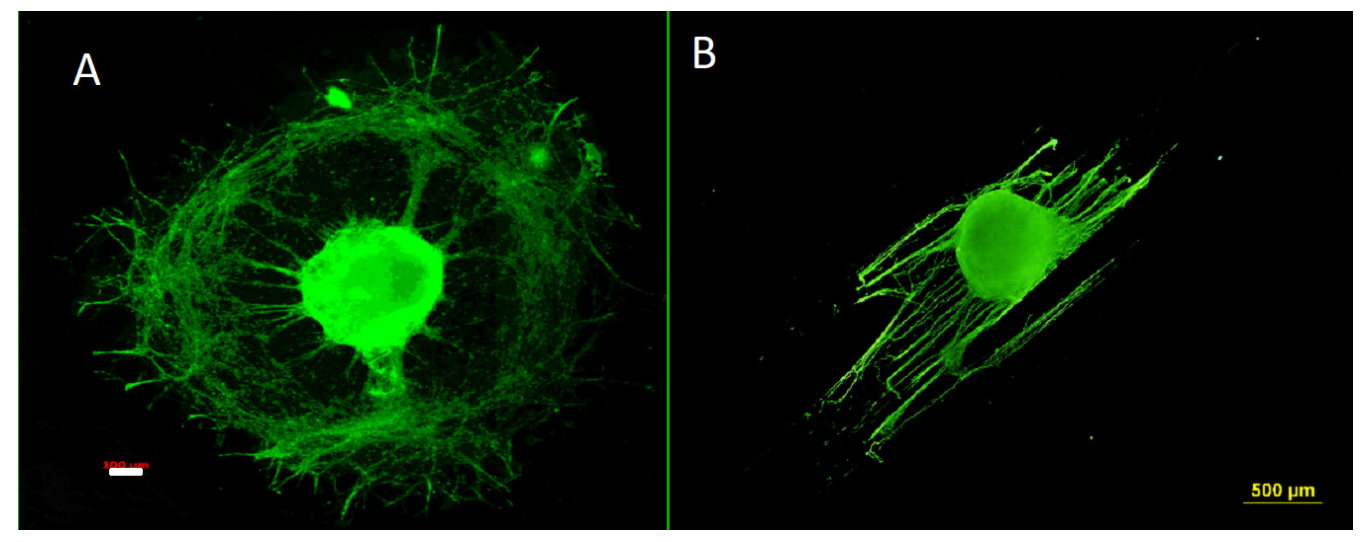

Figure 2-4: Chick DRG cell cultures. (A)Cell cultured without topographical cues (B)Cell cultured on aligned fiber scaffold 

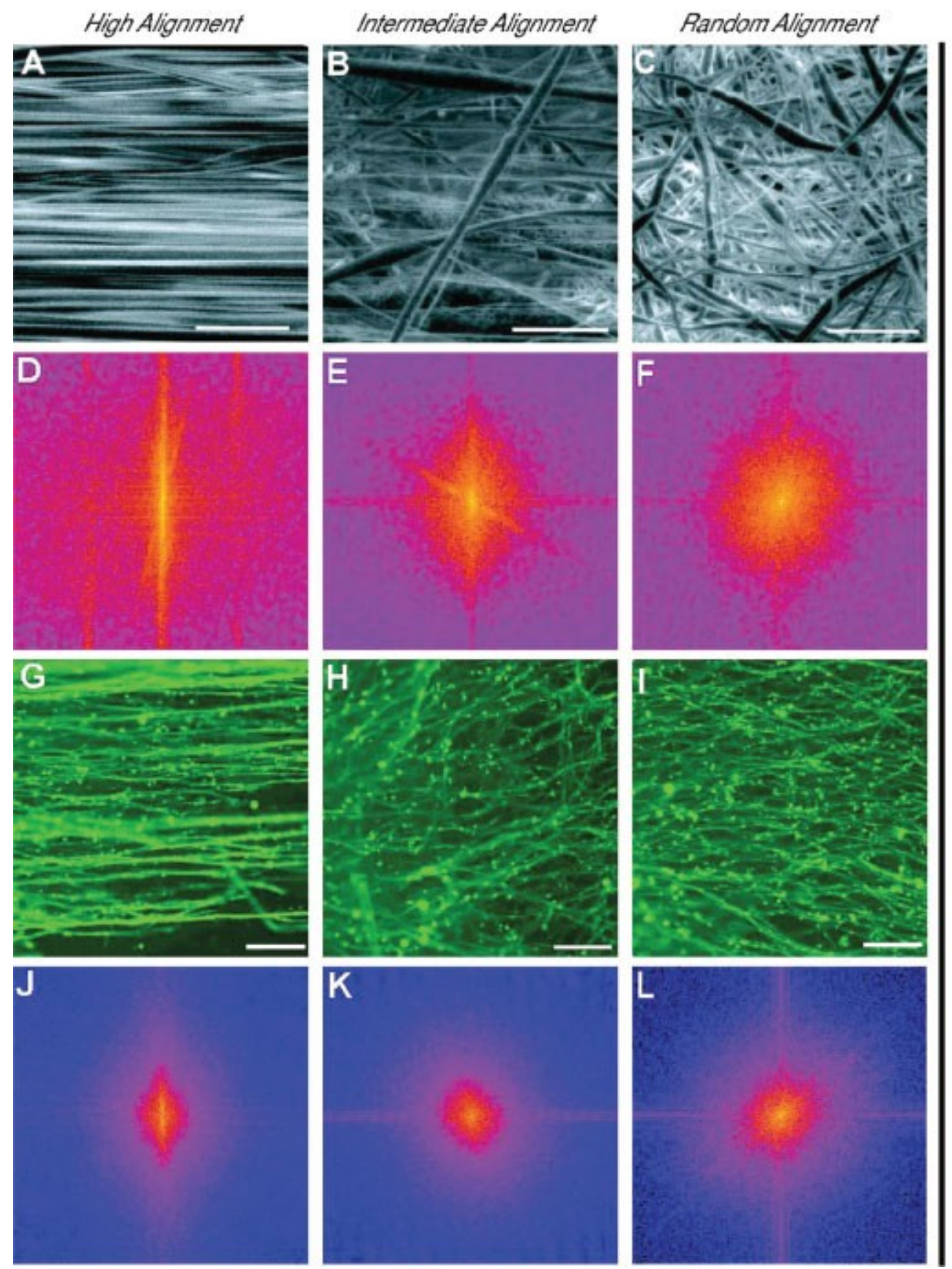

Figure 2-5: A-C: SEM images of various alignment of electrospun PLLA fibers. D-F:

FFT images from the SEM images in A-C. G-I: Neurite outgrowth from DRG cells. J-

L: FFT images from the images in G-I. Reused with permission from Wiley (Appendix 

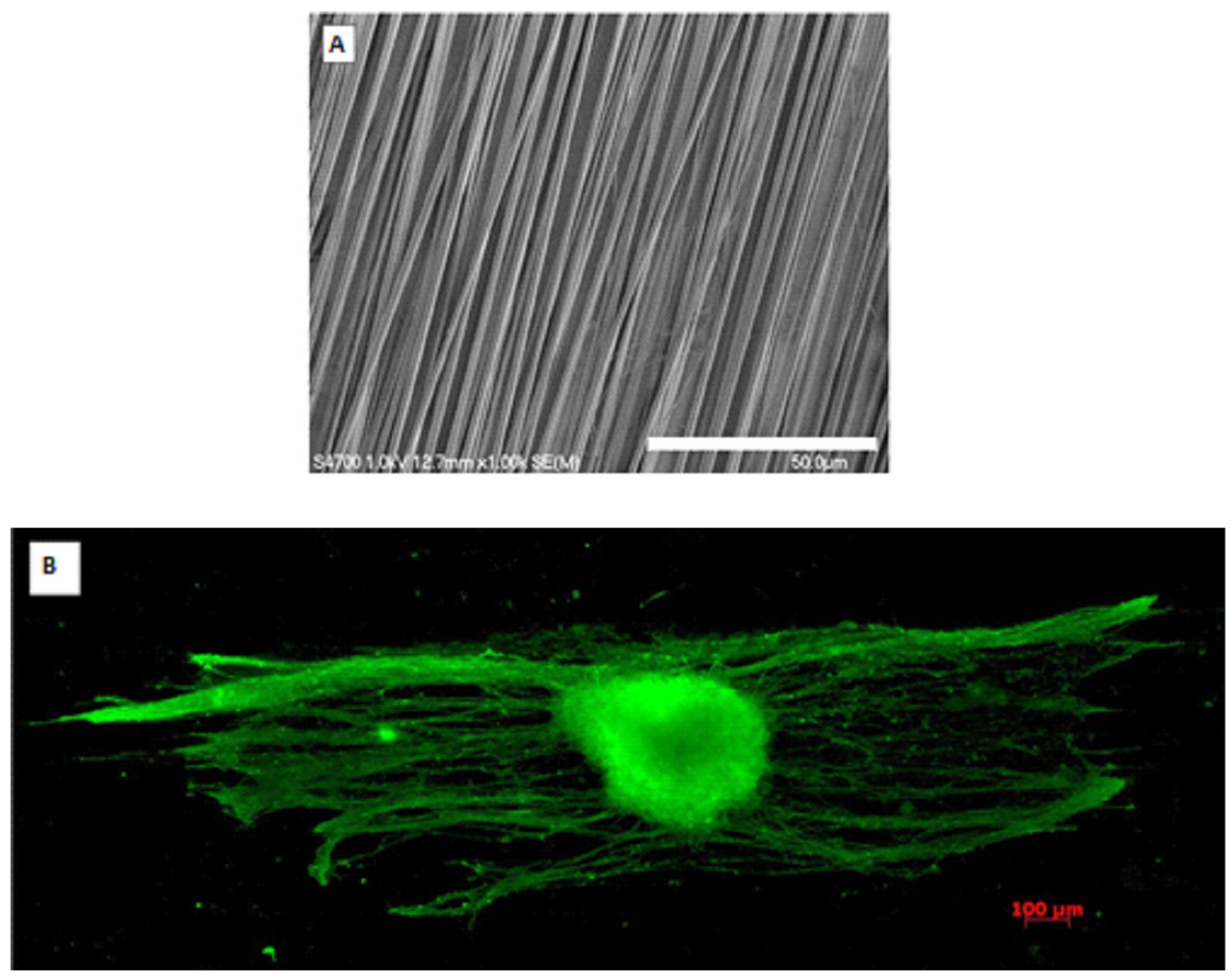

Figure 2-6:(A) Highly aligned electrospun PLLA nanofibrous mat. (B) Stage E9 chick dorsal root ganglia (DRG) showing axonal extension along fiber alignment. The aligned fibers aided in highly extensive axonal outgrowth. Reused with permission from IOP Science (Appendix 8.3)

While a rotating drum or wheel is the most common technique used for creating aligned scaffolds ${ }^{3,20,34,52-55}$, some researchers have found other methods to achieve aligned fiber mats. Jing et al. used parallel, conductive copper plates to achieve parallel aligned poly(propylene carbonate) fibers ${ }^{56}$. The electrostatic interactions between the 
positively charged polymer strand and the conductive copper plates allowed for the fibers to stretch across the gap between the plates in an aligned fashion. They achieved aligned fiber mats using this set-up, with $95 \%$ of fibers being $\pm 20^{\circ}$ of the direction of alignment ${ }^{56}$. Zhao et al. used a parallel electrode method to create aligned fiber scaffolds made of polyacrylonitrile ${ }^{57}$. The method the group used was meant to overcome the disadvantage of a rotating drum that limits the ability to collect a wide area due to geometric limits imposed by the size of the wheels and drums that are used. They modified their parallel electrode set-up by placing a positively charged ring in the space between the spinneret and the collecting electrodes to limit the area of whipping of the jet during electrospinning, thus allowing for more control during the electrospinning process $^{57}$. By using the ring to modify the parallel electrode set-up, the group increased the alignment of the fibers while also decreasing the diameter. The fiber diameter decreased from a majority of the fibers being around $250 \mathrm{~nm}$ in diameter to being around $150 \mathrm{~nm}$ in diameter, and the alignment increased by more than $20 \%{ }^{57}$. The improved topography makes the scaffolds more suitable for application ${ }^{57}$. Dippold et al. employed a similar technique to spin aligned fibers using parallel alumina rods ${ }^{58}$. The rods were grounded, and used to collect aligned PCL-collagen fibers. Bhutto et al. employed parallel magnets at either end of a very slowly rotating collector to collect highly aligned poly(L-lactide-co-caprolactone)(P(LLA-CL)) fibers ${ }^{59}$. They used two strong magnets, a south pole magnet at one end of the collecting rod and a north pole magnet at the other end, to create the aligned fiber scaffolds. 


\subsubsection{Aligned scaffolds in other tissue engineering applications}

Aligned fiber scaffolds have also been employed in other tissue engineering applications to improve the compatibility of the fibers. The use of fiber mats in cardiovascular engineering to increase their mechanical properties and their interactions with the target cells has been explored. Xu et al. demonstrated that by using an aligned structure of nanofibers they were able to observe more favorable interactions between smooth muscle cells (SMCs) and the scaffold, and the alignment of the fibers provided growth of cells in a specific direction and increased adhesion ${ }^{60}$. Xu et al. demonstrated that aligned fiber scaffolds, when used with the chemical signals provided by a proprietary bioglass material providing ionic products, on the fibers, could increase the signaling between bone marrow cells and endothelial cells ${ }^{61}$. They spun scaffolds that were a mixture of poly(D,L-lactide) (PDLLA) and polycaprolactone (PCL), and achieved aligned scaffolds by collecting the fibers on a roller that was rotating at a speed of $2000 \mathrm{rpm}^{61}$. The expression of vascular endothelial growth factor (VEGF) in a co-culture of human bone marrow stromal cells (HBMSCs) when cultured on aligned fiber mats with bioglass with human umbilical vein endothelial cells (HUVECs) was increase four times $^{61}$. The combined structural and chemical signaling also increased the junctional communication between the HBMSCs and HUVECs.

\subsubsection{Alternative polymers and blends for electrospun neural scaffolds}

Using mixed polymeric solutions may have some benefits in tissue engineering scaffolds, such as increased biocompatibility. For example, a composite solution may be 
made with a biodegradable, synthetic polymer and a natural biopolymer such as a protein. By combining the two types of polymers one can achieve increased biocompatibility of the scaffold while also capitalizing on the mechanical properties of a biodegradable synthetic polymer. Kijenska et al. showed that by spinning poly(L-lactic acid)-co-poly( $\varepsilon$ caprolactone) (P(LLA-CL)) with collagen I and collagen III protein they could make the fibers more hydrophilic when compared to spinning just P(LLA-CL), and thus more biocompatible ${ }^{7}$. They also showed that cell proliferation was higher during in vitro cultures on the composite scaffolds. While adding collagen to the fibers did slightly decrease the tensile strength, that was offset by spinning aligned fiber mats using a rotating steel disc at $1500 \mathrm{rpm}^{7}$. Aligning the fibers also increased the cell proliferation during in vitro cell cultures using neonatal mouse cerebellum stem cells ${ }^{7}$. A nerve guidance scaffold made from poly(serinol hexamethylene urea)/polycaprolactone blend (PSHU/PCL) solution electrospun as nanofibers was studied by Jenkins et al. ${ }^{62}$. The scaffold was modified with microchannels to mimic nerve topography and conjugated with a short peptide specific to cell binding to mimic biochemical cues. The scaffolds were seeded with human neural stem cells (hNSCs), and were found to increase hNSC survival and differentiation compared to a pure PCL control conduit ${ }^{62}$. Cells migrated and extended axons into the PSHU/PCL modified scaffolds, but did not show this behavior on the PCL conduits. Cells proliferated along the full length of the PSHU/PCL conduit by 14 days $^{62}$.

While electrospun fibers have shown the ability to aid axonal extension of in vitro DRG cells and proliferate stem cell lines, it has been difficult to duplicate that success in 
the complex environment of the neural system in vivo. Kakinoki et al. demonstrated nonaligned fiber scaffolds formed into hollow conduits on rabbits in vivo. They tested the action potential of the injured nerves repaired with nerve guide conduits (NGCs) made from electrospun PLLA-laminin nanofibers against those of a healthy nerve. None of the injuries repaired with NGCs reached near the active potential of healthy nerve tissue ${ }^{26}$.

Jiang et al. investigated the use of PCL NGCs in a peripheral nerve injury in rats ${ }^{28}$. Aligned PCL fiber mats were electrospun using a rotating target at a speed of 2,200rpm and then rolled into conduits. For comparison purposes, PCL films were fabricated by compressing PCL solution into a flat film. The films were then rolled into a conduit ${ }^{28}$. The in vivo tests were performed by placing a conduit, either consisting of PCL fibers or PCL film, into a transected sciatic nerve of a rat. The results showed that the rats who received fibrous PCL grafts experienced a higher degree of recovery than the rats who received a graft made from the PCL film. However, no rat experienced full recovery and the recovery was still lower than the recovery observed when using autografts ${ }^{28}$.

Most in vitro tissue cultures are performed in a two-dimensional environment, with fibers spun on a flat coverslip or glass surface, and then the cells are placed on the flat fiber surface. However, the spinal cord and in vivo environment are three-dimensional. Very few researchers have performed in vitro cell studies on fiber mats that are freestanding, not lying on a flat surface, and thus in a three-dimensional environment. The substrate in the two-dimensional environment of in vitro studies may also support neurite outgrowth. Spinning the fibers to create unsupported mats may remove the 2-D restraint of the axonal outgrowth of supported fibers. If axons grow between the small gaps that 
occur between fibers in the mats, the axon would extend along the flat surface, and be forced to grow outward along the channel between the aligned fibers. This phenomenon traps the axons in the channel even if they are not truly adhered to the fiber surface. If cells are cultured on fibers that are spun elevated from the surface, the environment becomes three dimensional, and the axons are not constrained to the channels between the fibers. Xie et al. performed cell studies on free-standing, suspended, aligned fiber mats using $\mathrm{PCL}^{39}$. The in vitro DRG cell culture results performed by their group did indicate that neurites followed the alignment of the free-standing nanofibers well with some minor deviations from fiber alignment.

The surface properties of the fibers, such as smoothness and porosity, have also been shown to impact cell growth during culture. Schaub et al. showed that the surface of electrospun PLLA nanofibers can be altered from smooth to rough by changing the solvent mixture of the electrospinning solution ${ }^{36}$. Regular, smooth fibers were electrospun from a solution of PLLA in a chloroform and dichloromethane solvent mixture. To determine whether the topography of the fibers affected cell cultures, a base solution of PLLA in chloroform was prepared. Then, various solvents were added to different amounts of the base solution. Water, ethanol and dimethyl sulfoxide (DMSO) solutions in chloroform base were all made and used for electrospinning ${ }^{36}$. Changing the solvent mixture affects the drying time of the fibers and the viscosity of the solution, which in turn can alter the surface of the fibers. In some cases, rapid evaporation of the solvent can cause the surface of the polymer to break down into solvent rich regions, which when dried cause roughness in the surface of the fiber ${ }^{36}$. In the study by Schaub, 
different solvents created different surface textures on the fibers. Water produced small, round depressions. Ethanol produced small but elongated depressions. Adding DMSO to the electrospinning solution caused large, more distinct depressions to form. The fibers with large depressions were tested for cell interaction. The group found that when cells were cultured on fibers containing large depressions, they do not elongate and spread the way a cell does when cultured on a smooth fiber surface, thus indicating that fiber topography can alter cell interactions with the fibers ${ }^{36}$

\subsection{Complex Electrospinning Techniques}

\subsubsection{Coaxial fibers for polymer scaffolds in tissue engineering}

The spinning of coaxial fiber scaffolds is a more complex extension of electrospinning techniques. By using a dual-spinneret, coaxial nanofibers can be electrospun using different core and sheath materials. Coaxial fibers have many advantages in tissue engineering applications. This geometry may allow incorporation of different pharmaceuticals, growth factors or polymers into the core component of the fibers to further enhance their effect on neural tissue regeneration ${ }^{63-65}$. The incorporation of proteins to promote growth or the biocompatibility of the scaffold into the core of a coaxial fiber protects the proteins from denaturation in the harsh solvent environment during electrospinning ${ }^{66}$. By placing drugs or growth factors into the core of the fibers, the polymer sheath guards against burst release and keeps the drugs active in vivo longer, as they are released based on the slower degradation rate of the polymer sheath ${ }^{66}$. Coaxial

fibers can also be spun in aligned mats similar to single-stranded fibers, further enhancing the abilities of the fibers to direct axonal outgrowth. 
Kuihua et al. demonstrated that by encapsulating nerve growth factor (NGF) into the core of aligned coaxial fibers, they improved the in vivo results, showing increased nerve regeneration in the sciatic nerve of rats. Encapsulating NGF into the core of polymeric coaxial nanofibers decreased the degradation rate of the NGF and caused a slower, more consistent release to occur. However, the results did not show complete regrowth, and did not include tests to show functional recovery of the injured leg ${ }^{44}$. Su et al. showed that drug release in vitro experienced a much more stable, steady release profile rather than the initial burst release and then drop-off profile of drugs spun in mixed solution as a single layer fiber ${ }^{67}$. A steadier drug release profile from coaxial fibers makes them more suited for in vivo applications, since the scaffold must remain viable in vivo long enough to assist complete tissue regrowth, which can take weeks. If the drugs encapsulated in the fiber scaffolds are released and degrade too quickly, they are not able to support sustained tissue growth.

Nguyen et al. used coaxial electrospinning techniques to spin fibers with a salicylic acid and poly(ethylene glycol) core and a porous PLLA sheath ${ }^{68}$. The study compared the drug release profile of salicylic acid released from the core at different core feed rates of $0.1 \mathrm{~mL} / \mathrm{h}, 0.2 \mathrm{~mL} / \mathrm{h}$, and $0.4 \mathrm{~mL} / \mathrm{h}$, and compared the release of the drug from porous and non-porous sheath fibers ${ }^{68}$. The study found that as the feed rate of the core increased, so did the initial burst release of the drug due to an increase in drug being present. Nonporous fibers had a lower burst release due to a decrease in membrane transfer of the drugs $^{68}$. Jalaja et al. spun coaxial fibers of a chitosan shell and a gelatin core to examine the possible uses for the fibers in biomedical applications ${ }^{65}$. The group spun randomly 
oriented fibers with an average diameter of $150 \pm 60 \mathrm{~nm}$. Samples were cross-linked with dextran aldehyde, sucrose aldehyde or glutaraldehyde. Cell proliferation of human osteoblast-like cells on the fiber samples was comparable to the proliferation of the control on a TPS plate during in vitro culture ${ }^{65}$.

Zhang et al. explored the use of coaxial fibrous structures with a silk-fibroin sheath and using brain-derived neurotrophic factor (BDNF) combined with vascular endothelial growth factor (VEGF) for peripheral nerve repair ${ }^{55}$. They explored putting BDNF in the sheath with the silk fibroin and VEGF in the core, as well as reversing the placement of the two different growth factors. They used a rotating drum covered with aluminum foil rotating at 2000rpm to achieve aligned fiber mats ${ }^{55}$. The average diameter achieved for the fibers was $2.5 \pm 0.7 \mu \mathrm{m}$, and most of the fibers were from 0 to $30^{\circ}$ from the axis of alignment ${ }^{55}$. At three days of in vitro cell culture of Schwann cells, both types of mats had increased proliferation as compared with controls, and at seven days the scaffolds that contained VEGF in the core and BDNF in the sheath achieved a higher rate of proliferation than the scaffolds with BDNF in the core and VEGF in the sheath ${ }^{55}$. It was thought that the increased release of BDNF in the sheath aided proliferation. Studies for in vivo results were also performed. Rats received a nerve injury in the pelvic nerve. Rats that received the two different types of scaffolds with growth factor both had a higher percentage of nerve fiber content and vascularization as compared with rats that received a scaffold containing only silk fibroin fibers. The scaffolds that contained VEGF in the sheath and BDNF in the core had a higher rate of vascularization and innervation than the other growth factor-containing scaffolds ${ }^{55}$. The VEGF in the sheath of the fiber meant a 
rapid release of vascular growth factor, thus vascularization occurred more quickly. The faster rate of vascularization aided in a higher degree of innervation. The coaxial structure did prove more beneficial than the single-stranded, aligned silk-fibroin fibers.

Wang et al. spun aligned core-shell fibers with an NGF/PEG core and a poly(DLlactide-co-glycolide) (PLGA) shell ${ }^{69}$. Aligned fibers were achieved by collecting on a rotating drum rotating at 4000rpm. The average diameter of the fibers was $513 \pm 174 \mathrm{~nm}$, larger than that of plain PLGA fibers with a diameter of $299 \pm 67 \mathrm{~nm}^{69}$. Studies were performed in vivo to examine the effect of the scaffolds on a sciatic nerve injury in rates. The PLGA and the PLGA/NGF scaffolds were compared to autografts. After 12 weeks of implantation, the PLGA/NGF scaffolds achieved nerve conduction velocities (NCV) similar to those observed with the autograft, the current standard of peripheral injury repair. The NCV of the PLGA/NGF scaffolds was $46.8 \pm 2.9 \mathrm{~m} / \mathrm{s}$, while for the autograft it was $49.05 \pm 1.3 \mathrm{~m} / \mathrm{s}$ and for the PLGA scaffold it was $26.9 \pm 2.9 \mathrm{~m} / \mathrm{s}^{69}$. The scaffolds containing coaxial fibers with an NGF core were much more effecting in functional recovery after injury. The same phenomenon was observed during the muscle weight test in the rats. The weight ratio was 0.61 for the PLGA/NGF scaffold, 0.64 for the autograft and 0.48 for the PLGA scaffolds ${ }^{69}$. The coaxial structure allowed for a steadier release of NGF from the core and aided in recovery.

Gluck et al. demonstrated that by spinning a sheath of composite PCL/gelatin and a core of polyurethane in a random mat that they increased the mechanical properties of the coaxial fibers as compared to single layer, traditional fibers. The Young's modulus of the coaxial fiber scaffolds was found to be $93.36 \pm 9.11 \mathrm{MPa}$ as opposed to $76.80 \pm$ 
14.66Mpa for single stranded fibers. The tensile strength was also improved in the coaxial mat, being $1.67 \pm 0.47 \mathrm{MPa}$ for the coaxial mats versus $0.83 \pm 0.25 \mathrm{MPa}$ for mats with single-stranded fibers. These improved mechanical properties make them more suitable for the biological, in vivo environment ${ }^{70}$.

\subsubsection{Application of conducting polymer fibers in scaffolds for neural tissue engineering}

The axons in neural tissue transmit information via an electrical signal. The electric potential of the nervous system is provided by high salinity within the tissue. Sodium, chloride, and potassium ions are all found in high concentrations, sodium and chloride being on one side of the cell and potassium being on the other side. The distribution of the opposite ions causes a positive potential on one side of the cell and a negative potential on the other side ${ }^{71}$. Given the electrical nature of neural tissue, one possible way to increase the affinity of neural tissue to electrospun fiber scaffolds is to make them conductive using conductive polymers. It has been demonstrated that electrical stimulation has the ability to enhance neuronal extension when applied across the growth media $^{72-77}$, and it has been demonstrated that even when repair of the injury is delayed, electrical stimulation can still positively affect neural tissue regrowth ${ }^{78,79}$.

Electrical stimulation of the culture well imitating spontaneous bursts during neuronal maturation of retinal progenitor cells (rPCs) resulted in decreased expression of $\mathrm{N}$-cadherin and increased $\beta$ III-tubulin expression on both a protein and gene level, indicating differentiation into retinal neurons, as well as calcium ion signaling correlating to functional action potential firing ${ }^{80}$. Human neural progenitor cells (nHPCs) 
preferentially differentiate into neurons on electrospun piezoelectric polyvinylidene fluoride-trifluoroethylene (PVDF-TrFE) scaffolds, identifying the benefits of electrical in addition to physical cues by demonstrating higher neuronal preference on scaffolds with higher piezoelectricity ${ }^{81}$. Adams et al. demonstrated that by supplying electrical stimulation across a collagen gel they could improve neurite extension ${ }^{73}$. By subjecting E11 chick DRG cells cultured on a collagen gel to an electrical field of $24 \mathrm{~V} / \mathrm{m}$ combined with NGF, neurite lengths were measured as being significantly longer than cells cultured only in the presence of NGF. Lengths of neurites on cells subjected to stimulation were $159.6 \pm 11.0 \mu \mathrm{m}$, while the neurite length of cells with only NGF present were $120.1 \pm$ $11.4 \mu \mathrm{m}^{73}$. Chang et al. also demonstrated that providing electrical stimulation in addition to NGF improved neurite outgrowth ${ }^{75}$. Rat pheochromocytoma stem cell line (PC12) cells were seeded on collagen and subjected to a field of either $50 \mathrm{mV} / \mathrm{mm}, 100 \mathrm{mV} / \mathrm{mm}$ or $200 \mathrm{mV} / \mathrm{mm}$ and were also treated with NGF, then compared to cells that were only treated with NGF. They found that the average length of neurites of cells treated with NGF for 46 hours was $76 \mu \mathrm{m}$, while cells treated with NGF and electrical stimulation for 46 hours had an average neurite length of $110 \mu \mathrm{m}^{75}$.

A major obstacle to using conductive polymers in neural tissue scaffolds is the lack of biocompatible and biodegradable conductive polymers. Some research has focused on the use of two: poly(3,4-ethylenedioxythiophene) (PEDOT) and polypyrrole (PPY), often doped with poly(styrene sulfonate) (PSS). The dopant improves the electrical properties of the chemical and often aids in solubility. PPY has been used in many biological electrical applications ${ }^{82-87}$. It has high conductivity ${ }^{82,83}$, is easy to prepare from the 
pyrrole monomer ${ }^{83-88}$, is biocompatible when tested ${ }^{83,85-88}$, and is easily modified through surface modification techniques ${ }^{83,87}$. These characteristics make PPY attractive to use in electrically conducting tissue scaffolds. However, PPY has an extremely slow biodegradable rate, in some cases taking months to degrade, and tends to make brittle fibers when produced via electrospinning techniques ${ }^{87}$. It also loses its conductive properties too quickly over time ${ }^{89,90}$. Yamato et al. found that after only sixteen hours of experiencing a constant applied voltage of $400 \mathrm{mV}$ that PPY:PSS films lost all of their electrical activity ${ }^{89}$. PPY has defects in the structure of the polymer that limit the electrical response and ultimately lead to a breakdown in the conductive properties ${ }^{90}$. PEDOT, on the other hand, is highly conductive $\mathrm{e}^{91-94}$ and maintains its conductive properties over a much longer period than $\mathrm{PPY}^{89,90}$. Yamato tested the electrochemical stability of PEDOT:PSS films at the same conditions as the PPY:PSS films, and found that even after eighty hours of exposure to a voltage field of $400 \mathrm{mV}, 76 \%$ of the conductive activity of the film was still present ${ }^{89}$. It is also found to be biocompatible $\mathrm{e}^{92,93,95}$.

Sebaa et al. tested the effects of PEDOT coatings on magnesium degradation in vivo for medical applications, as well as the biocompatibility of the PEDOT with stem cells cultured in vitro. Magnesium is being explored for biological implants because of its biocompatibility and conductivity, but it has a very high degradation rate in vivo. The group found that coating magnesium particles with PEDOT increased the stem cell viability as compared to uncoated magnesium nanoparticles ${ }^{92}$. Luo et al. cultured fibroblast cells on PEDOT-coated indium-tin-oxide (ITO) substrates in vitro and found 
through assay studies that cell viability was greater than $90 \%$ and much higher than the viability of cells cultured on plain ITO films ${ }^{93}$. In addition, in vivo implantation of PEDOT-coated glass substrate showed no immune reaction or immune cell infiltration ${ }^{93}$. Asplund et al. found that human neuroblastoma cells cultured in vitro on PEDOT materials showed no signs of abnormal cell deat ${ }^{95}$. The group also demonstrated that increasing the amount of PEDOT did not lead to a negative correlation in cell viability ${ }^{95}$.

Researchers have used other methods to combine the benefits of conductive polymers with the more complex structure of core-sheath fibers to produce more complex fiber structures as tissue scaffolds. Xie et al. demonstrated that by electrospinning polymeric nanofibers made from PCL or PLLA solutions and then coating with PPY, that they could combine the mechanical properties of polymeric, single-strand nanofibers with the electrical properties of PPY. The in vitro results showed that the neurites of DRGs extended further on the conductive fibers ${ }^{96}$ (Figure 2-7). Neurites from DRG cells had a maximum length of $1723 \pm 339 \mu \mathrm{m}$ on aligned fiber structures as opposed to $946 \pm$ $164 \mu \mathrm{m}$ o random fiber scaffolds ${ }^{96}$. However, when applying a current to conductive coresheath random fibers, the maximum axonal length was actually longer than that observed with aligned fibers without axonal stimulation $(1733 \pm 141 \mu \mathrm{m})^{96}$.

Zhang et al. used coaxial spinning techniques to spin both aligned and random $\mathrm{P}(\mathrm{LLA}-\mathrm{CL}) / \mathrm{SF}$ composite fibers with conductive polyaniline (PANI) polymer as the sheath and P(LLA-CL)/SF with NGF as the core. In vitro cell cultures performed with rat PC12 cells demonstrated a longer neurite growth along the fibers when electrical stimulation was applied ${ }^{97}$. Xu et al. utilized emulsion electrospinning techniques to spin 
PVA/EDOT solution with a special solvent concentration such that when the solution is spun and the fibers dry a core-shell structure results with EDOT monomer in the core ${ }^{98}$. Emulsion electrospinning is a method which can be used to spin core-sheath fiber structures. It involves using a mixture of two solvents which are not miscible in each other. One solvent is present in a continuous phase and the other solvent is present as droplets $^{22}$. When the solution is electrospun, the resulting fibers have a core-sheath structure with the continuous phase encapsulating the droplet phase ${ }^{99}$. In the case of the study by Xu et al., PVA was the continuous phase and thus the sheath of the fiber, and the EDOT monomer was the drop phase forming the core. Once the fibers were spun, the monomer was then polymerized using chlorine gas to achieve a PEDOT core ${ }^{98}$. In the Xu et al. study their core-shell fibrous structures were examined for polymerization of the PEDOT core using FTIR and UV-Vis spectroscopy techniques. The FTIR showed bands associated with carbon double bonds and sulfoxy-groups, indicating the presence of PEDOT as opposed to the monomer. The coaxial fibers also exhibited good conductive properties when measured with cyclic voltammetry ${ }^{98}$. However, the study did not include cell culture research. 

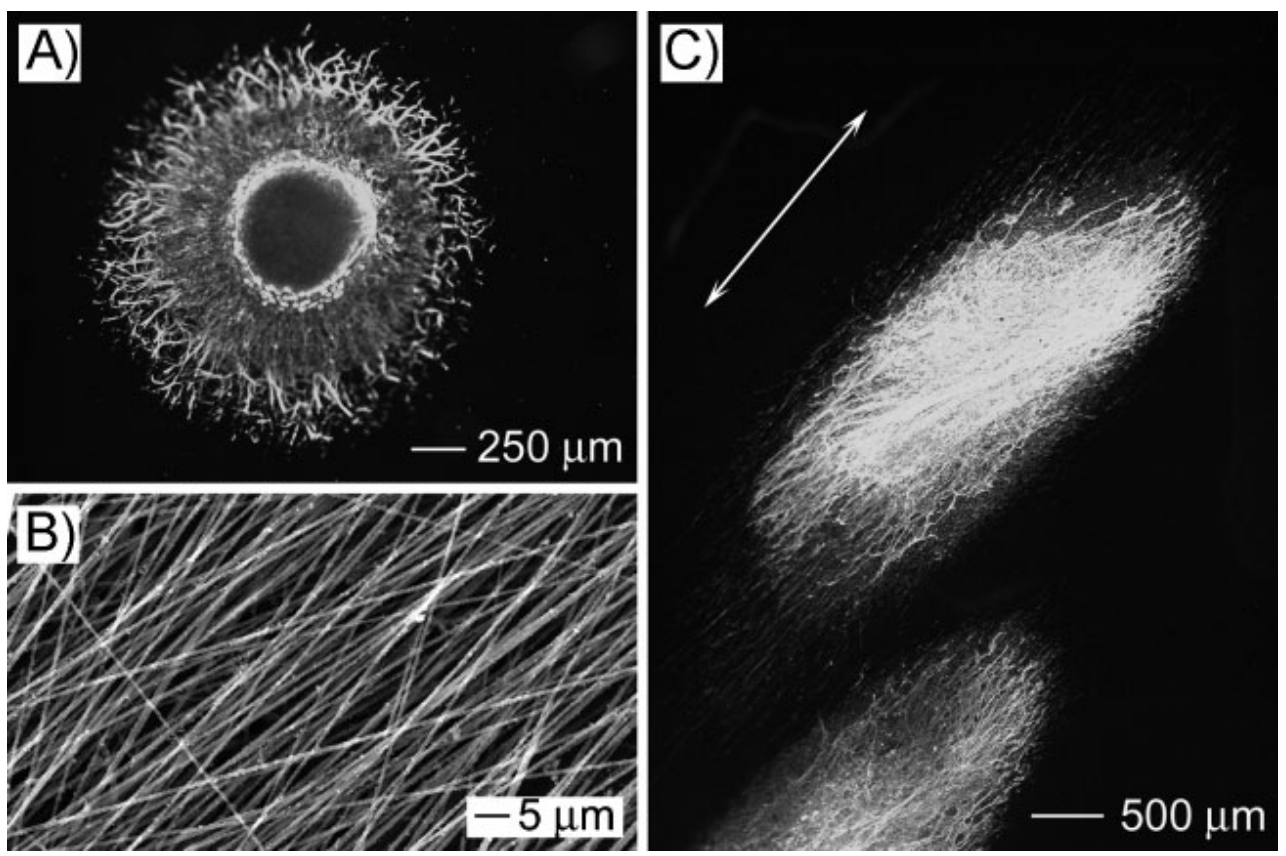

Figure 2-7: (A) Micrograph of DRG neurite outgrowth field on random polymer fiber mat with PCL-PPY coaxial fibers. (B) Aligned PCL-PPY coaxial fibers. (C) Micrograph of DRG neurite outgrowth field on aligned PCL-PPY coaxial fibers. Reused with permission from Wiley (Appendix 8.3).

\subsection{Future Outlook for Electrospun Neural Scaffolds}

Electrospinning techniques for neural applications have been extensively studied to produce nano-and microfiber scaffolds for neural tissue engineering applications. In vitro studies using embryonic DRGs have shown impressive results in terms of axonal growth and guidance; however, corresponding success using in vivo models has not been achieved. Hurtado et al. demonstrated impressive neural tissue regeneration in an in vivo model of a transected rat spinal cord, but the results did not indicate significant functional recovery ${ }^{20,100}$. Their work showed that extensive axonal regeneration occurred at the 
rostral end, but not to the same extent at the distal end. Although phenomenologically extensive axonal growth and regeneration is observed, the mechanisms for this process are not adequately understood. To achieve success in vivo, the growth and guidance cues provided by aligned fiber scaffolds need to be systematically examined. These cues could include textural/morphological effects, surface chemistry and cell adhesion, biochemical signals, environmental factors including associated support cells, or electrical/ionic stimulation. The surface texture and mechanical properties of the fibers may inhibit or enhance axonal outgrowth, but it is unclear how this works with the fiber surface chemistry to achieve the optimal cell growth. Biochemical signals can be controlled in vitro by dosage, but in vivo, careful control is difficult to achieve. Coaxial fibers used for neural engineering allow for the direct incorporation of biochemical factors and by tailoring the sheath properties, can provide a method for controlled, extended release. This is an exciting research opportunity in the area of neural tissue engineering. Similarly, electrical stimulation has been shown to promote axonal growth, and fibers with either a conducting core or sheath may provide an in situ method for applying the stimulus directly to the scaffold. The use of such conducting fibers has shown promise in further improving axonal outgrowth in vivo, as demonstrated by Xie et al. By encasing these conductive fiber cores within an insulating sheath, one can effectively create an artificial axon which can be implanted directly in vivo.

As with most tissue engineering approaches, a combined strategy for in vivo neural tissue regeneration will probably be required to achieve clinical success. For example, Martin et al. are examining combining the structural cues of aligned PLLA microfibers 
with direct electrical stimulation via a core/shell structure with an insulating sheath and a conductive polymer core. A similar approach can be taken for controlled release of biochemical agents by using a porous sheath material tailored to the desired release rates with a core material impregnated with the desired active ingredient. The sophistication of nano-engineered fibrous scaffolds is rapidly evolving, and the application of these new structures in vivo one of the most promising approaches for resolving PNS and CNS injuries. 


\section{Electrospinning Aligned, Free-Standing PLLA Microfiber Scaffolds on Elevated Stages for 3D In Vitro Cell Culture ${ }^{2}$}

\subsection{Abstract}

Electrospinning is a technique that has been studied as a method to produce fiber scaffolds as a repair strategy for spinal cord injuries (SCIs). In this chapter, aligned, freestanding fiber scaffolds using poly-L-lactic acid (PLLA) were developed as an in vitro model to study cell interaction on free-standing fiber scaffolds in vivo. Stages were designed to allow for the formation of free-standing fiber scaffolds that were not supported by an underlying surface. The elevated stages had column heights of $3 \mathrm{~mm}$ and the columns were $1 \mathrm{~cm}$ apart. Fibers were spun across the columns of the stages to produce free-standing fiber scaffolds. Fiber analysis of the free-standing scaffolds showed the fibers were fairly aligned with a $2 \sigma$ of $16.8^{\circ}$ and $22.2^{\circ}$. Average diameters were $4.4 \pm 2.7 \mu \mathrm{m}$ and $4.6 \pm 2.1 \mu \mathrm{m}$. Fiber densities were $162.5 \pm 17.1$ and $85.0 \pm 25.2$ fibers $/ \mathrm{mm}$. The scaffolds were then used for in vitro cell culture using chick dorsal root ganglia (DRG). Fiber scaffolds were also spun on a flat substrate and used for in vitro cell studies for comparison. The average axonal outgrowth of the cell grown on fibers with an underlying support was $833 \pm 204 \mu \mathrm{m}$. The axonal outgrowth for the cells grown

\footnotetext{
${ }^{2}$ The material within this chapter will be submitted for review for publication. The journal has not yet been chosen.

Citation:

Martin, Rachel, Wendling, Marie, Qian, Zichen, Zhao, Feng and Mullins, Michael. "Electrospinning Aligned, Free-Standing PLLA Microfiber Scaffolds on Elevated Stages for 3D In Vitro Cell Culture."
} 
on free-standing fiber scaffolds was $522 \pm 113 \mu \mathrm{m}, 649 \pm 320 \mu \mathrm{m}$ and $1100 \pm 221 \mu \mathrm{m}$. The axonal outgrowth observed for DRG cells cultured on free-standing fiber scaffolds was comparable to those grown on fibers with an underlying surface, indicating that cells follow the alignment of fibers even without an underlying support. This serves as an in vitro model for in vivo cell behavior with free-standing fiber scaffolds and shows the ability of aligned fiber scaffolds to aid in axonal outgrowth.

\subsection{Introduction}

Injuries to the central nervous system (CNS) are among the most debilitating, since the complex nature of neural tissues results in poor regeneration, leading to lifelong disabilities ${ }^{1}$. Injuries to the CNS include spinal cord injuries (SCI), with over 17,000 new SCIs occurring in the United States each year ${ }^{2}$. This significant problem has led to the exploration of alternative methods for enhancing nerve tissue regrowth. When axons are severed, glial scar tissue forms at the site of the injury. Since new axons cannot grow through the scar tissue, this prevents axonal outgrowth and reconnection of the severed nerves. One strategy to overcome this is a growth-permissive scaffold across the scar to guide axons during extension, allowing them to reconnect over the scar along the scaffold.

One promising method for tissue scaffold construction is electrospinning polymer micro- and nanofiber structures that provide guidance for tissue growth. Tissue applications that have been explored include bone ${ }^{48,49,101}$ cardiac $^{60,102}$, cartilage $^{47,103}$ and neural tissues $3,7,15,28,33,35,42,43,104$, using a variety of polymers. Polymers used include synthetic polymers like polycaprolactone (PCL), poly-lactide-co-glycolic acid (PLGA), 
polylactic acid (PLA), as well as natural polymers such as collagen and silk fibroin and various blends of polymers. Electrospun fiber scaffolds can provide guidance cues for cell growth in tissues, particularly for axonal outgrowth of neurons, where the scaffolds act as a bridge across glial scar tissue in SCIs. Aligned poly-L-lactic acid (PLLA) fiber scaffolds have proven successful in facilitating aligned, structured outgrowth of axons in chick dorsal root ganglia (DRG) during in vitro cell studies ${ }^{34,35}$. Aligned fiber scaffold conduits implanted in rat in vivo injury models showed significant regrowth of the injured spinal cord section; however, significant improvements in hind limb motor function were not observed, hindering the clinical use of fiber scaffolds for SCI repair ${ }^{20,100}$.

One explanation for insufficient recovery in vivo is that in previous in vitro studies, the fibers were electrospun on a flat substrate, and the subsequent in vitro cultures thus performed on this flat surface ${ }^{34,35}$. This substrate restricted axonal outgrowth to a two-dimensional plane, which is different from the three-dimensional in vivo environment. For the flat substrate, the axons could be growing in the spaces or channels between the fibers, and not truly attaching to the fibers themselves. To investigate this explanation, a free-standing fiber scaffold was created on an elevated culture stage to examine the axonal outgrowth in vitro but without the restriction of the substrate surface. Thus, if the extending axons are not truly attaching to the fibers themselves, they can detach and grow out of the plane of the scaffold, or simply fall off. This is more similar to the environment of implanted scaffolds in vivo. Our approach is to create a method for collecting free-standing electrospun fibers unsupported by a surface, thus providing a three-dimensional in vitro tissue culture environment. This would help to determine if the 
aligned fibers are providing the growth cues for axonal outgrowth that was observed in vitro rather than a constricted two-dimensional surface. It is our hypothesis that the axons adhere directly to the fiber surface and take directional growth cues from the fiber alignment. Therefore, removing a supporting surface for the fibers will not affect axonal outgrowth, and that the axons will continue to align and extend along the free-standing fibers. An experimental model to test this hypothesis was accomplished by creating a fiber collection stage with columns at a predetermined height and electrospinning fibers that collected across the top of the columns. The collected fibers are suspended in the culture media above the surface of the stage, and by using chick DRG cells, comparative in vitro studies between the free-standing and the surface supported fiber scaffolds were performed.

\subsection{Materials and Methods}

\subsubsection{Materials}

All materials were used as purchased. Poly-L-lactic acid (PLLA) was obtained from NatureWorks LLC. Chloroform was used as the polymer solvent in all cases (Sigma Aldrich). The elevated stages were obtained through two methods. Some of the stages were manufactured using 3D-printing and made from poly-lactic acid (PLA). Some of the stages were manufactured using conventional machining methods and were made from acrylic tubing.

\subsubsection{Spinning Stage Design}

Some of the elevated stages were manufactured using 3D-printing and were made from poly-lactic acid (Figure 3-1). The rest of the stages were machined from acrylic 
square tubing (10mm I.D., $12.5 \mathrm{~mm}$ O.D.). The tubing was cut into $10 \mathrm{~mm}$ long pieces and then sliced in half lengthwise to have the same footprint as the 3D-printed stages, allowing both to fit in a 6-well culture plate. In mock-ups to determine the appropriate column height and distance between the columns, prototypes were made using plastic coverslips as the base for the stage and attaching various widths of plastic perpendicular to the base using superglue. Column heights tested were $2 \mathrm{~mm}, 3 \mathrm{~mm}$ and $4 \mathrm{~mm}$. During electrospinning tests, it was determined that the $4 \mathrm{~mm}$ tall columns created too much air movement when attached to the rotating wheel. This caused the fiber strand to whip around erratically and impeded the formation of highly aligned scaffolds. The $2 \mathrm{~mm}$ tall columns were not high enough to keep the fibers elevated over the surface. Threemillimeter columns prevented sagging of the fibers with an acceptable degree of air movement. In the final design, a distance between the columns of $10 \mathrm{~mm}$ was used. This minimized the sagging of the fibers to the surface while allowing enough surface area for implanting cells during tissue culture. 

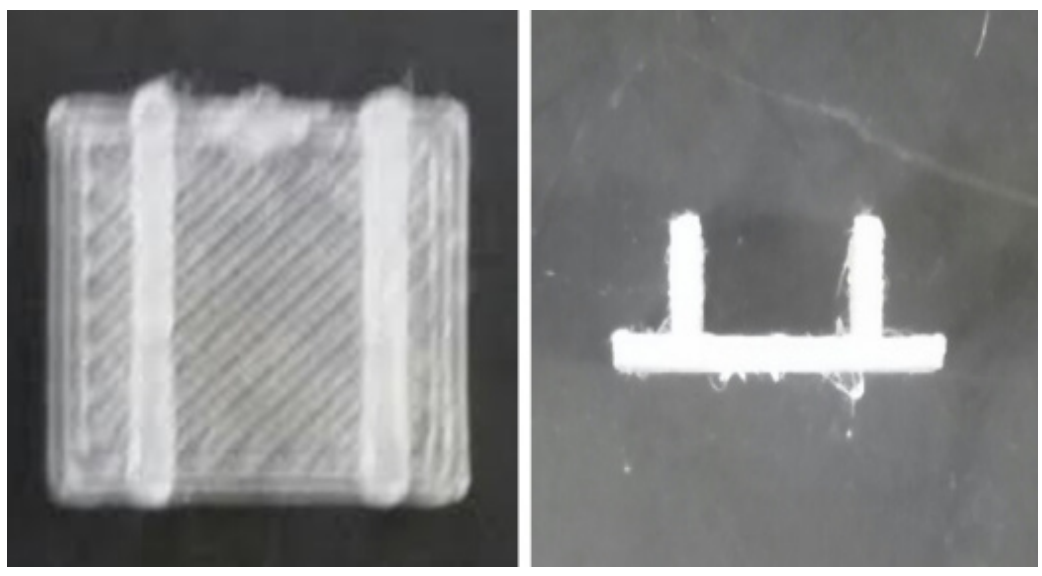

Figure 3-1: 3D-printed electrospinning stages

\subsubsection{Electrospinning}

The electrospinning and fiber collection methods used are detailed in a previous paper by the authors ${ }^{105}$. For this study, an $8 \mathrm{wt} \%$ PLLA solution in chloroform was used as the electrospinning media. To prepare the solution, $10 \mathrm{~mL}$ of chloroform was added to $1.29 \mathrm{~g}$ PLLA in a sample vial with a stir bar. The vial was sealed with Parafilm ${ }^{\circledR}$ and placed on a warm stir/heat plate for approximately one hour or until the PLLA was completely dissolved. The solution was drawn into a syringe fitted with a 16-gauge needle and placed on a syringe pump set to $0.9 \mathrm{~mL} / \mathrm{hr}$. Tygon $®$ tubing connected the syringe to a 22-gauge blunt spinning needle. A short piece of tubing was placed over the end of the needle to insulate it and prevent stray currents. The 22-gauge blunt spinning needle was placed $10 \mathrm{~cm}$ above a spinning aluminum wheel that acted as the grounded collection surface. This distance was determined by the drying time for the fibers generated at the tip of the needle. The further the distance, the more likely the solvent 
will evaporate and a dry sample will be produced. The wheel was rotated by a motor set to $1250 \mathrm{rpm}$. The wheel had a diameter of $12 \mathrm{~cm}$ and a width of $1.2 \mathrm{~cm}$. The entire apparatus is inside a Plexiglas chamber allowing for control of the spinning environment. It is necessary to control the humidity and temperature during electrospinning to ensure the desired fiber diameter and quality are consistently achieved. The chamber also restricts harmful vapors and allows for increased safety during the procedure. A Glassman high-voltage power supply was connected to the spinning needle and set to positive $17 \mathrm{KV}$. High-powered halogen lamps were used to illuminate the box, and a microscopic video camera allowed for clear visual imaging of the fiber being spun. The images were sent to a small TV monitor and collected digitally for later analysis. Formation of a Taylor's cone can be observed to ensure proper spinning is occurring (Figure 3-2).

During electrospinning, the solution is pumped to the spinning needle. The droplet formed at the tip of the needle is pulled into a micron-sized fiber by the voltage difference between the needle and the grounded aluminum wheel. The fibers are collected on the stages or flat $10 \mathrm{~mm}$ by $10 \mathrm{~mm}$ coverslips attached to the wheel. The coverslips are attached to the wheel using double-sided Scotch tape (3M) and the stages are attached to the wheel using a heavier double-sided mounting tape. Spinning times varied, but usually lasted between 10 and 30 minutes. Once spinning was complete, the samples were removed from the wheel and imaged, then air-dried prior to cell cultures. 

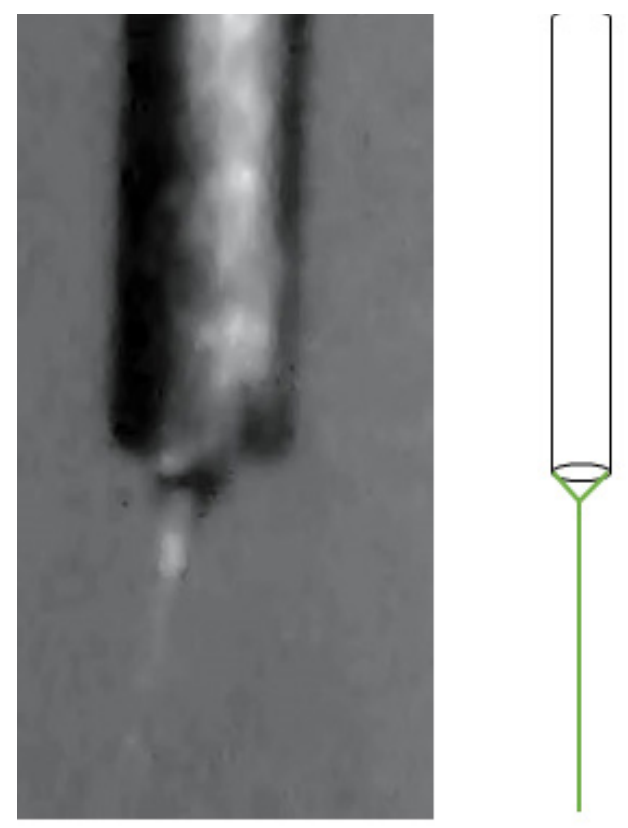

Figure 3-2: Taylor's cone observed. right-hand image: captured during electrospinning. left-hand image: drawing

\subsubsection{Fiber Analysis}

Fibers were analyzed using optical microscopy and ImageJ software to determine the alignment and diameter of the fibers. Images were taken of the fibers using an Olympus BX-15 optical microscope with a camera attachment and X-Cite 120 Fluorescence Illumination System. The images were digitally analyzed using ImageJ software $(\mathrm{NIH})$. The DiameterJ ${ }^{\circledR}$ plug-in available for ImageJ was used to determine fiber diameter and degree of alignment. Alignment of fibers was determined using the OrientationJ ${ }^{\circledR}$ function via Fast Fourier Transform (FFT) analysis. The results are reported as the angle deviation from perfect alignment. 


\subsubsection{Dorsal Root Ganglia (DRG) Isolation and Culture}

Cell cultures were performed in accordance with the Michigan Technological University Institutional Animal Care and Use Committee (IACUC). Stages and coverslips prepared with fiber samples were sterilized using an ethylene oxide sterilization chamber. Samples were placed in a sterilization bag with a vial of ethylene oxide. The bag was purged of air, the vial broken, and the samples placed in the chamber for 12 hours.

Chick dorsal root ganglia (DRG) cells were isolated from stage E9 chick embryos. The eggs were incubated at $37^{\circ} \mathrm{C}$ for nine days prior to isolation. The incubator was filled with distilled water supplemented with water bath cleaner. The water level was checked regularly and more distilled water added when necessary. Once the DRGs were isolated, they were placed via Pasteur pipette on top of the sterile fiber samples in 6-well plates. The wells were filled with neurobasal medium supplemented with $10 \mathrm{ng} / \mathrm{mL}$ mouse 2.5S native nerve growth factor (Thermo Fisher Scientific, Waltham, MA). Samples were incubated for 3-5 days, with the medium being changed every 48 hours.

\subsubsection{Immunochemistry Staining}

After culturing was complete, the cells were treated and stained for imaging. First, the culture media was removed from the wells and the samples were fixed in a formalin solution of $3.7 \%$ formaldehyde in PBS buffer for 30 minutes. The fixing solution was 
removed, and cells rinsed twice with PBS buffer. Cells were then blocked with 1\% BSA in $50 \mathrm{~mL}$ of fixing buffer. The cells were blocked for one hour to prevent protein binding for the immunochemistry staining. After the blocking was complete, the buffer was removed and $50 \mu \mathrm{L}$ solution of primary antibody TUG 1 in a ratio of 1:200 in blocking buffer was added to the cells. The samples were placed in the refrigerator with the antibody overnight. The culture plates were wrapped with Parafilm ${ }^{\circledR}$ to prevent the cells from drying out. The next day the samples were removed from the refrigerator and the antibody solution drained. The cells were rinsed three more times for 5 minutes each with $1 \mathrm{~mL}$ of blocking buffer. The secondary antibody, goat anti-mouse IgG antibody, was then added to cells. The antibody was kept out of direct light. A 1:200 dilution of antibody in blocking buffer was made, and $100 \mu \mathrm{L}$ of solution as added to the samples. They were covered with aluminum foil and left to incubate at room temperature for 40 minutes. The samples were rinsed three times with $0.2 \%$ Triton in PBS to remove excess unbound antibodies. About $1 \mathrm{~mL}$ of solution was added to each well and each rinse was done for five minutes. The cells were then stained with DAPI to stain for cell nuclei. A 1:1000 dilution of DAPI in PBS was made and 1mL was added to each well. The samples sat at room temperature for 10 minutes and then rinsed one time with PBS. The samples were mounted onto cover slides with Mountant and sealed with nail polish. They were left to dry for 30 minutes and then imaged using fluorescence optical microscopy.

\subsubsection{Statistical Analysis}

Statistical testing was performed on the axonal outgrowth of the in vitro cell cultures. Minitab ${ }^{\circledR}$ was used to calculate a Tukey's test to determine if there was any 
statistical differences between the samples. The Tukey's test compared the average outgrowth of the different samples with each other and grouped them based on statistical similarity.

\subsection{Results}

\subsubsection{Fiber analysis}

Images of the fiber samples, both on the stages and on flat coverslips, were obtained using the optical microscope with a digital camera attachment. Every sample was imaged after collection to characterize the fibers. Characteristics such as dryness, alignment, diameter and density of fiber scaffolds were determined using the microscope. Figure 3-3 shows fiber scaffolds collected both on flat coverslips and on elevated stage collectors.

All the fiber scaffolds were aligned to some degree. To quantify this, Fast Fourier Transform (FFT) analysis using Image ${ }^{\circledR}$ was performed on the fibers to determine their degree of alignment. Samples spun on flat coverslips had a $\sigma=5.1^{\circ}\left(2 \sigma=10.2^{\circ}\right)$, indicating $95 \%$ of the fibers were within about $10^{\circ}$ of the axis of alignment. The fibers in Figures $3-3 \mathrm{~A}$ and $3-3 \mathrm{~B}$ for the elevated stages had a $\sigma=8.4^{\circ}\left(2 \sigma=16.8^{\circ}\right)$, indicating that $95 \%$ of the fibers were within about $17^{\circ}$ of the axis of alignment. Some other unsupported fiber scaffolds used for DRG cell culture were not as highly aligned (e.g. Figure 3-7) with a $\sigma=11.1^{\circ}\left(2 \sigma=22.2^{\circ}\right)$, indicating that $95 \%$ of the fibers were within about $22^{\circ}$ of the axis of alignment. The approximate average fiber density of the scaffold in Figure 3-3A and 3-3B was $162.5 \pm 17.1$ fibers $/ \mathrm{mm}$. The approximate average fiber density of the other unsupported scaffolds used for DRG cell cultures was $85.0 \pm 25.1$ 
fibers $/ \mathrm{mm}$. The approximate average fiber density of the scaffold spun on the flat substrate was $397.5 \pm 40.3$ fibers $/ \mathrm{mm}$. These results are summarized in Table 3-1. Analysis was also performed to determine the number of fibers that were within $1^{\circ}, 2^{\circ}$, and so on of alignment. The data was plotted and can be observed in Figure 3-4. The frequency plots for the alignment analysis were also generated and are shown in Figure 35.

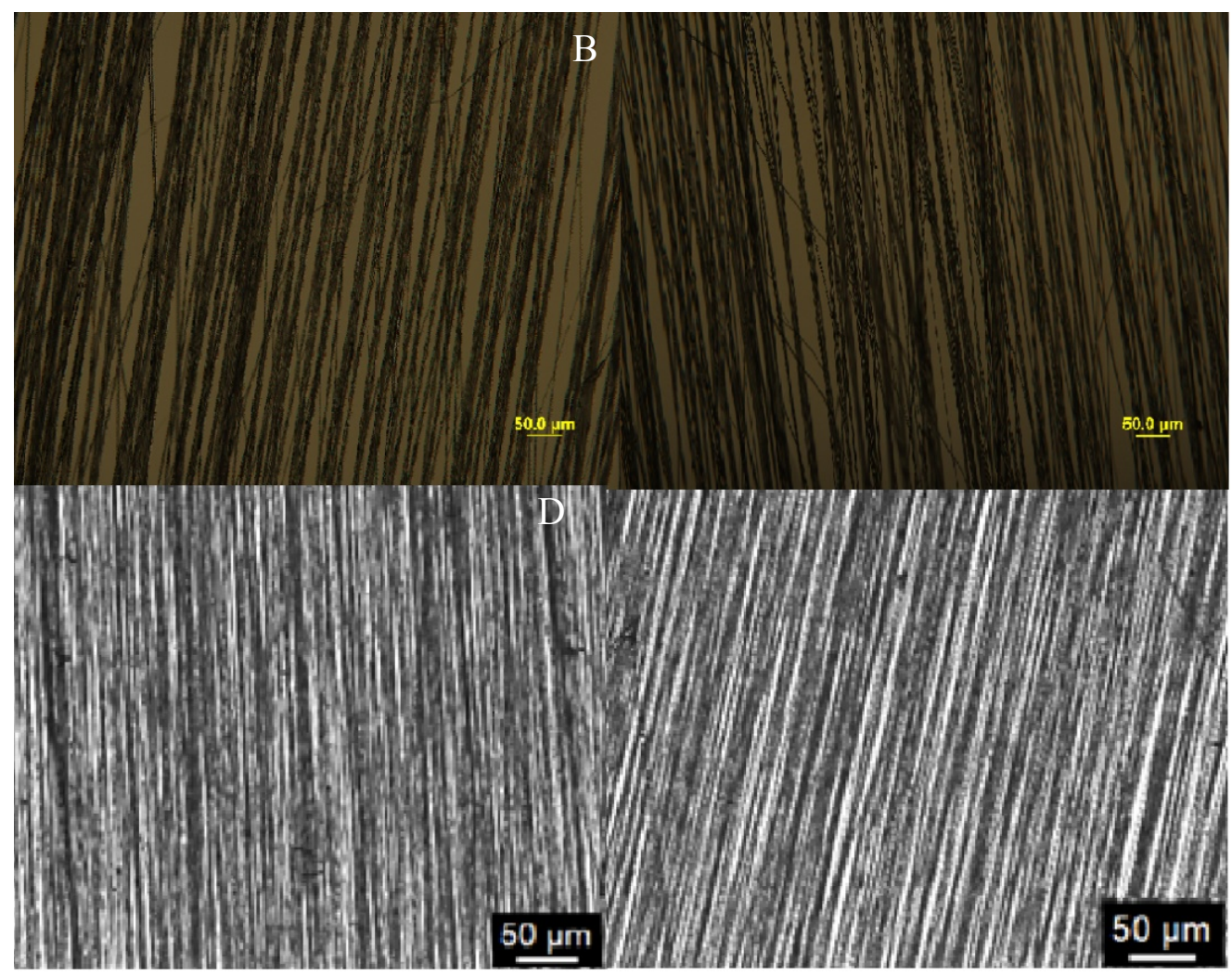

Figure 3-3: Optical microscope images of electrospun PLLA fibers. Spinning time for samples $A$ and $B$ was $32 \mathrm{~min}$ with a voltage of $17 \mathrm{KV}$ and a flow rate of $0.9 \mathrm{~mL} / \mathrm{hr}$. The fibers were spun on 3D stages with $3 m m$ columns. $C$ and $D$ show fibers spun on flat coverslips at a spinning time of $23 \mathrm{~min}$ with a voltage of $17 \mathrm{KV}$ at a flow rate of

\section{$1.05 \mathrm{~mL} / \mathrm{hr}$.}


Table 3-1 FFT Analysis of fiber alignment, fiber diameter and fiber density

\begin{tabular}{|c|c|c|c|}
\hline $\begin{array}{c}\text { Sample } \\
\text { Conditions }\end{array}$ & $\mathbf{2 \sigma}$ (degrees) & $\begin{array}{c}\text { Average Fiber } \\
\text { Diameter( } \boldsymbol{\mu m} \pm \text { ) }\end{array}$ & $\begin{array}{c}\text { Average Fiber } \\
\text { Density(fibers } / \mathbf{m m} \pm \text { ) }\end{array}$ \\
\hline $\begin{array}{c}\text { fibers directly on } \\
\text { flat substrate }\end{array}$ & $10.2^{\circ}$ & $2.8 \pm 1.3$ & $400 \pm 40$ \\
\hline $\begin{array}{c}\text { fibers across } \\
\text { columns, 32min } \\
\text { spinning time }\end{array}$ & $16.8^{\circ}$ & $4.4 \pm 2.7$ & $162.5 \pm 17$ \\
\hline $\begin{array}{c}\text { fibers across } \\
\text { columns, 23min } \\
\text { spinning time }\end{array}$ & $22.2^{\circ}$ & $4.6 \pm 2.1$ & $85 \pm 25$ \\
\hline
\end{tabular}

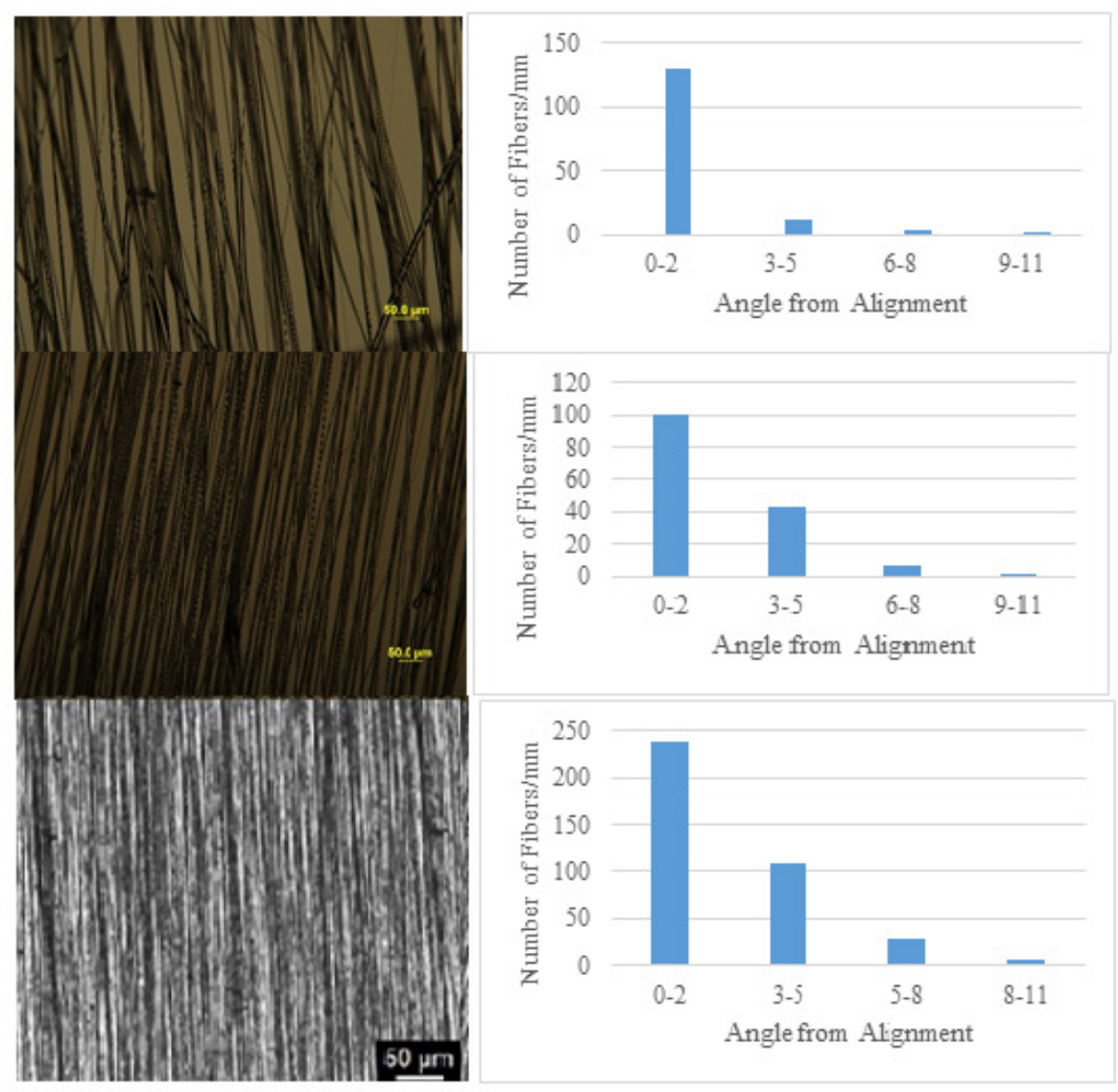

Figure 3-4: Plotted number of fibers at certain degrees from alignment. Top: 23min

Middle: 32min Bottom: coverslip 

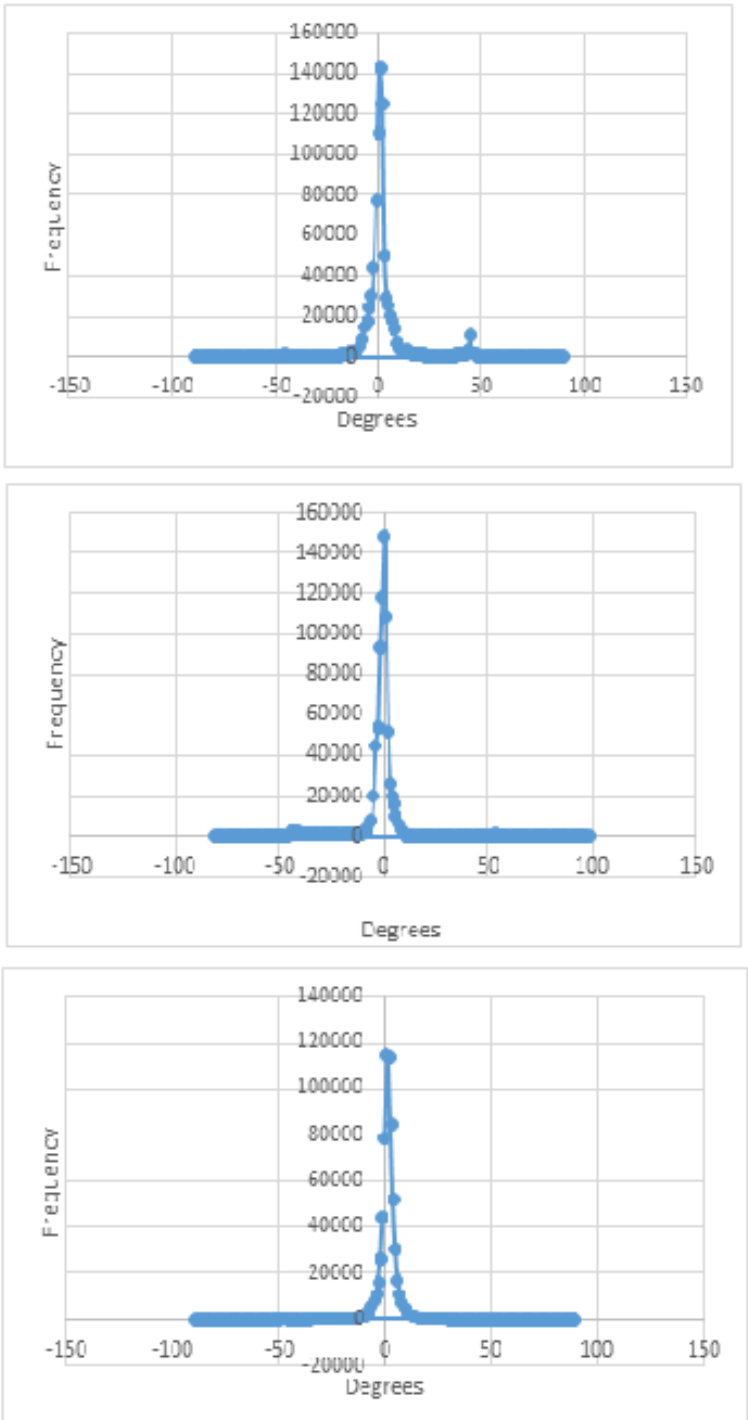

Figure 3-5: Frequency plots from alignment data. Top: 23 min Middle: 32 min Bottom:

$$
\text { coverslip }
$$




\subsubsection{DRG Cell Results}

Chick DRG cell cultures were performed on three different substrates: a flat coverslip without fibers, fibers spun directly onto a coverslip, and on fibers spun across the columns on the elevated stage. Figure 3-6A shows a DRG cultured on a flat coverslip without fibers. Without the topographical cues provided by the fibers, the axonal outgrowth becomes disordered and entangled, forming a wreath-like structure of dystrophic growth cones around the DRG. Figure 3-6B shows a cell culture performed on an aligned PLLA fiber scaffold spun directly onto a flat coverslip. With the guidance cues provided by the fibers, axonal outgrowth is aligned with the alignment of the fibers, indicating that the topology of the scaffold directs axonal outgrowth. Figure 3-7 shows 3day DRG cultures performed on scaffolds that were spun across the columns of the elevated stages. Even without the support of an underlying surface, the axonal outgrowth still follows the alignment of the fibers, and does not extend into or out of the plane of the elevated scaffold. Blue DAPI staining for cell nuclei shown in Figures 3-7B, 3-7D and 37F indicates that the axons are supported by glial cell proliferation. The axons in Figures 3-7A and 3-7B showed an average outgrowth of $649 \pm 320 \mu \mathrm{m}$. The axons of the cell in Figures 3-7C and 3-7D showed an average outgrowth of $1100 \pm 221 \mu \mathrm{m}$. The axons of the cell in Figures 3-7E and 3-7F showed an average outgrowth of $522 \pm 113 \mu \mathrm{m}$. The average axonal outgrowth of the cell in Figure 3-6B had an average outgrowth of $833 \pm$ $204 \mu \mathrm{m}$. The fiber samples in Figures 3-7A and 3-7E were spun for 32 minutes. The fiber sample in Figure 3-7C was spun for 23 minutes. These results are summarized in Table 32. As determined by the Tukey's test, the outgrowth of the sample in Figure 3-7A and 3- 
$7 \mathrm{C}$ were statistically the same, and the average outgrowth between the sample in Figure 3-7E and 3-6B were statistically the same. The sample in Figure 3-7B was statistically different from all of the samples.

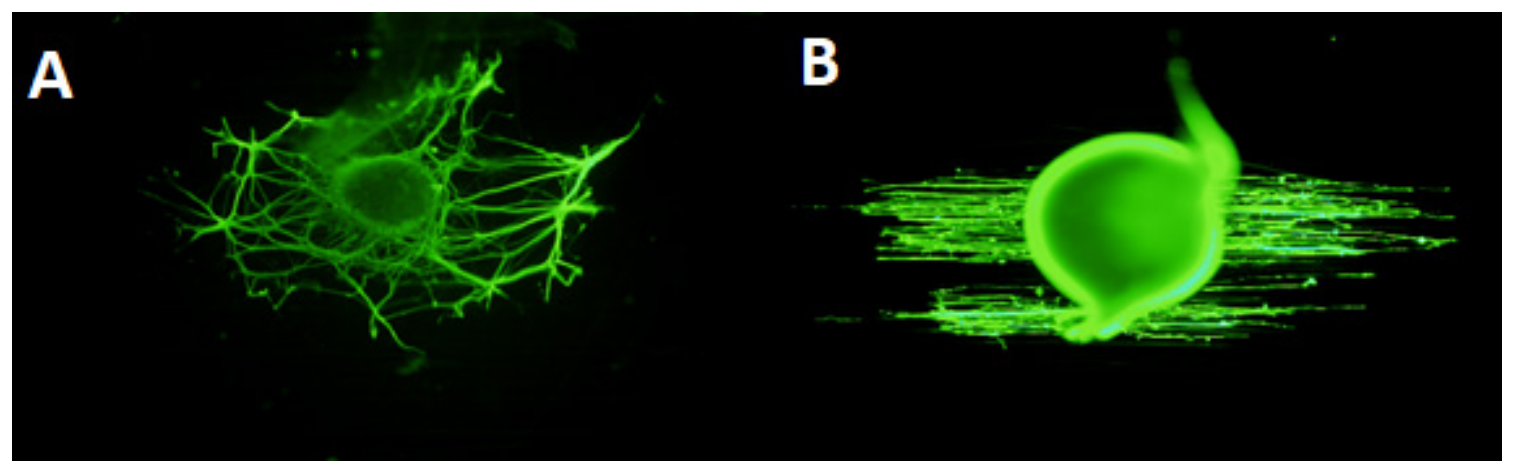

Figure 3-6 Immunochemical staining of 5-day chick DRG cultures on (A) coverslip (B) fibers. Without the topographical cues provided by the aligned fiber scaffolds, the axons become entangled in a wreath-like structure around the center of the cell and form dystrophic growth cones. The alignment of the fibers allows the axons to grow outward along the alignment of the fibers. 


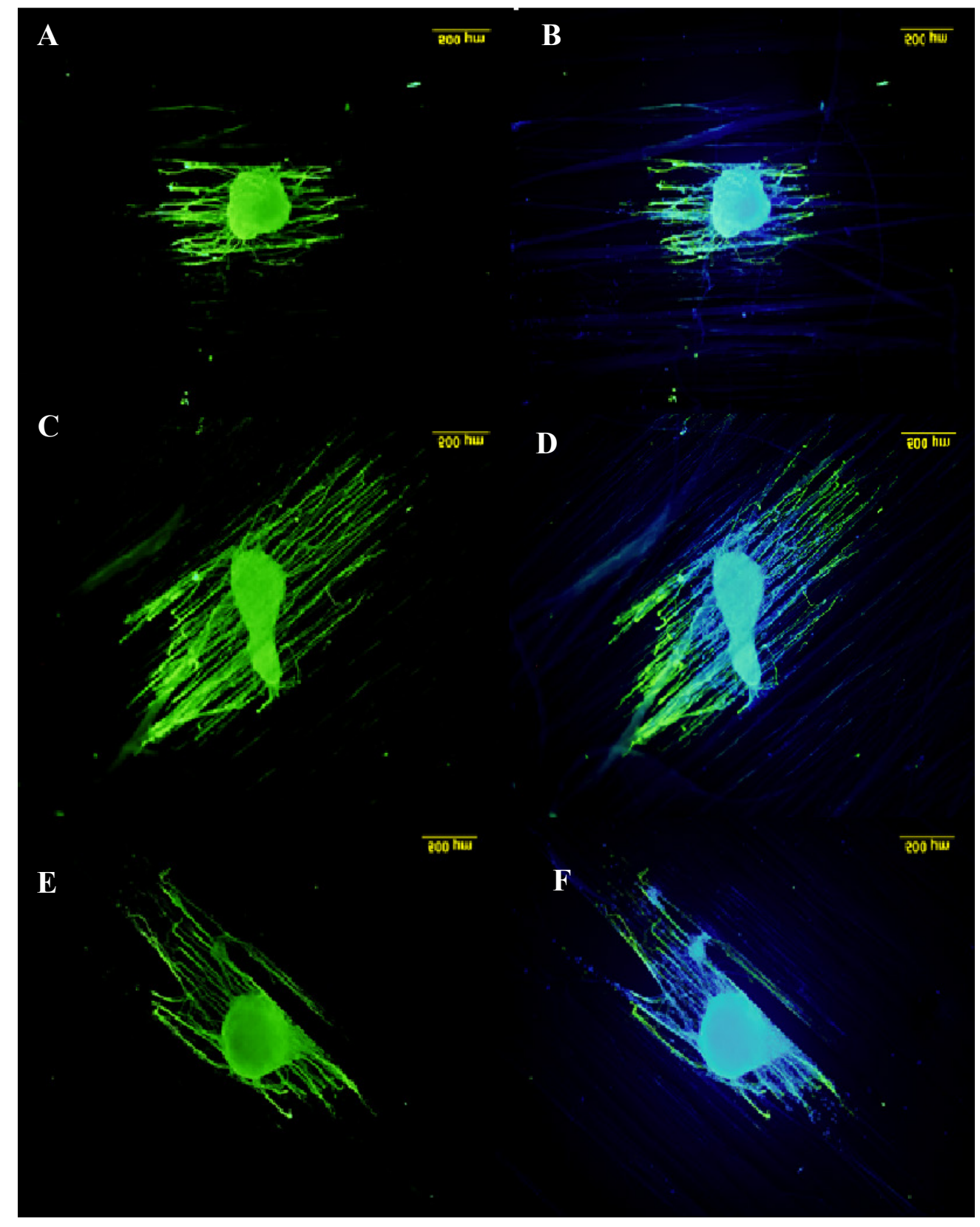

Figure 3-7 Immunochemistry staining of 3-day DRG cultures on free-standing fiber scaffolds. The aligned, free-standing fiber scaffolds aided in axonal extension despite lack of underlying, supporting surface. 
Table 3-2 Average axonal outgrowth for DRG culture study.

\begin{tabular}{|c|c|}
\hline Sample & Average Axonal Outgrowth \\
\hline 32-minute sample, 5A & $650 \pm 320 \mu \mathrm{m}$ \\
\hline 32-minute sample, 5E & $520 \pm 110 \mu \mathrm{m}$ \\
\hline 23-minute sample, 5C & $1100 \pm 220 \mu \mathrm{m}$ \\
\hline coverslip sample, 4B & $830 \pm 210 \mu \mathrm{m}$ \\
\hline
\end{tabular}

\subsection{Discussion}

Previously fibers were spun directly onto a grounded, metal electrode, in some cases free-standing but then transferred to a free-standing ring for cell culture ${ }^{39}$. To the best of the authors' knowledge, this paper outlines for the first time fibers directly spun on a free-standing, 3D support without being in direct contact with the grounded electrode. During electrospinning, critical factors including reduction of stray currents, minimizing air flow, achieving a strong enough attachment to the wheel, and the rotation rate of the wheel were considered through the control of different parameters. Using mounting tape, altering the height of the columns of the stages, controlling the humidity and achieving a wheel rotation speed that prevents detachment of the stages and minimizes airflow were all methods used to control electrospinning parameters. Fibers were successfully spun on the elevated stages. The fiber scaffolds produced in this fashion were of sufficient density, alignment and mechanical strength to provide a good support for cell culture. Figure 3-3A and 3-3B show fibers spun on elevated stages, while 
Figure 3-3C and 3-3D show fibers spun on elevated stages. In comparing the two different sets of electrospun fibers, the characteristics are extremely similar. The dryness and size of the fibers spun on the elevated stages as the fibers spun on a flat surface. The fibers spun on coverslips are more aligned than those spun on an elevated stage (Table 31). During electrospinning, due to the columns of the stages, a fan-like effect is caused when the wheel is spinning, which causes the fiber thread ejected from the end of the Taylor's cone to blow about more erratically than normal. This causes the fiber scaffolds to be less aligned, but the fibers are still sufficiently aligned for cell culture and do not exhibit crossing that inhibits axonal outgrowth.

Figure 3-6B shows the differences of culturing a DRG cell on a fiber scaffold to provide guidance cues versus a flat surface without such cues, as shown in Figure 3-6A. When a nerve cell is cultured on an untextured, flat surface, the axons extend a certain distance from the DRG before encountering each other, becoming entangled and eventually forming a wreath-like structure around the DRG. The entanglement of the axons indicates in the absence of external cues, useful extension of the axons may not occur. When the DRG cells are placed on an aligned fibrous mat, the axonal outgrowth is parallel to the fibers and in the direction of fiber alignment. Thus, aligned fiber scaffolds which provide guided axonal outgrowth may be beneficial in vivo in repairing SCIs.

A questions raised by the previous research ${ }^{34}$ related to the guidance mechanism for axonal outgrowth on the aligned fiber scaffolds. It was suggested that the guidance mechanism was due to axons growing in the channels between the fibers or between the fibers and the surface as opposed to the textural cues provided by the fiber surface. 
Because the mats were spun on a flat coverslip, the culture environment was constricted to the $2 \mathrm{D}$ plane. It is possible that during axonal outgrowth, an axon could grow in the interstitial space between fibers in the scaffold, or in the channel formed between two fibers on the surface. If the fibers are spun on free-standing scaffolds above the surface, this is similar to a $3 \mathrm{D}$ culture environment found in vivo. This would provide axons the opportunity to grow into and out of the plane of the fibers, and growth would be unrestricted by a supporting surface. To test this theory and determine the guidance mechanism of axonal outgrowth, a stage was designed that allows fibers to be spun across two columns of the stage, creating a scaffold suspended above the surface (Figure 3-1). This effectively creates a 3D growth environment for cell cultures.

As shown in Figure 3-7, DRG cells cultured for three days on the elevated fiber scaffolds continued to follow the axis of alignment of the fibers and did not grow in or out of the scaffold plane. Figures 3-7B, 3-7D and 3-7F show blue DAPI staining for cell nuclei. The blue stain shows the proliferation of glial cells, indicating that the axons are supported by glial cells, so applicable axonal outgrowth was achieved on the elevated stages. After three days of cell cultures, the samples in Figures 3-7A and 3-7C showed axonal extension that was greater than the width of the cell body. The cell in Figure 3-7A had a cell body with a length of $629 \mu \mathrm{m}$ and an average axonal length of $649 \pm 320 \mu \mathrm{m}$. The cell in Figure 3-7C had a cell body with a length of $437 \mu \mathrm{m}$ and an average axonal extension of $1100 \pm 221 \mu \mathrm{m}$. These two DRG samples showed extensive axonal outgrowth in only three days. While the DRG of Figure 3-7E had axonal extension less than the width of the cell body, the difference was within the standard deviation of the 
average axonal outgrowth. The DRG of that sample had a width of $558 \mu \mathrm{m}$ and an average axonal extension of $522 \pm 113 \mu \mathrm{m}$. The axonal outgrowth of the cell in Figure $3-$ $6 \mathrm{~B}$ was $833 \pm 204 \mu \mathrm{m}$. The length of axonal outgrowth of the DRG grown on fibers spun on a coverslip fell within the standard deviation of the axonal outgrowth of the cells grown on free-standing fiber scaffolds. The outgrowth of the samples in Figure 3-7A and Figure 3-7E was statistically the same, as was the outgrowth between Figure 3-7A and Figure 3-6B. Overall, the DRG samples grown on the free-standing fiber scaffolds showed impressive growth with limited hindrance due to the lack of underlying surface.

The average axonal outgrowth of the samples did not very greatly. The DRG in Figure 3-7C showed the greatest outgrowth, but the standard deviations of all three samples overlap, indicating the outgrowth was similar. The differences in axonal outgrowth can be explained by the differences in the fiber samples that were used in the culture process. Fibers that have more alignment, smaller diameter fibers or are more densely packed will allow for larger axonal extension. The DRGs in Figure 3-7A and 37E were seeded on fiber samples that were collected under the same conditions and were spun for the same length of time. The average diameter of those samples was $4.4 \pm$ 2.7 $\mu \mathrm{m}$. The DRG in Figure 3-7C was implanted on a fiber sample with an average diameter of $4.6 \pm 2.1 \mu \mathrm{m}$. The fibers from the cells in Figures 3-7A and 3-7E had a smaller average diameter, but a larger standard deviation and there were a higher number of larger diameter fibers than the sample from Figure 3-7C. Larger diameter fibers can be an impedance in axonal outgrowth ${ }^{35}$. While the samples in Figure 3-7A and 3-7E are more densely packed, before the fibers were completely dry some of them merged 
together to form larger fibers which may account for the lower axonal outgrowth length. The deviation may also be explained by the difference in fiber alignment. While the sample spun for 32 minutes had a higher overall degree of alignment with a $2 \sigma$ of $16^{\circ}$ as opposed to the $2 \sigma$ of $22^{\circ}$ for the sample spun for 23 minutes, the sample spun for 23 minutes had a higher number of fibers that were at a lower angle difference from the angle of alignment. Most of the fibers from the sample spun for 23 minutes were within $2^{\circ}$ of alignment, while the fibers from the sample spun for 32 minutes were much more distributed as far as their difference from the angle of alignment (Figure 3-4). The frequency plots in Figure 3-5 show that all the fibers centered around a reference point of 0 degrees from alignment.

The results from in vitro cell cultures on the free-standing fiber scaffolds indicates that the fibers are providing surface cues for axonal outgrowth, and axons prefer to adhere and follow the alignment of the fibers rather than grow unrestricted in and out of the scaffold plane. The surface of the aligned fiber scaffolds is the cause for the in vitro success seen previously, and provide a guidance mechanism for axonal outgrowth. These results also give clues as to how axonal outgrowth may occur along a bridge made of fiber scaffolds in vivo. The in vivo environment is not restricted to two dimensions, so cell cultures on the free-standing scaffolds may provide a better model of how axonal extension occurs in vivo.

\subsection{Conclusions}

The design of the elevated stages that can be used to produce free-standing fiber scaffolds was studied. Stages made from PLA with columns of $3 \mathrm{~mm}$ height and $1 \mathrm{~cm}$ 
distance apart were produced using a 3D printer, while other stages made from acrylic square tubing that produced scaffolds of the same dimensions were also used. The stages were attached to a rotating aluminum wheel of an electrospinning apparatus. Fibers made from PLLA were electrospun and collected across the top of the columns of the stages. Fibers were also spun onto a flat substrate for comparison purposes. The scaffolds were analyzed for average fiber diameter, degree of alignment and fiber density. The results of the fiber analysis performed are summarized in Table 3-1.

Chick DRG cell cultures were performed on both the free-standing scaffolds and the scaffolds on the flat substrate. Cell cultures were performed on free-standing scaffolds with different fiber densities and degrees of alignment to compare the axonal outgrowth between the two sets of scaffolds. Here it was shown that aligned fiber scaffolds can be spun on an elevated stage, providing a 3D environment for in vitro tissue culture. It was also demonstrated that in vitro DRG cell cultures performed on these elevated surfaces produce results similar to those seen previously. The average axonal outgrowth of the DRG cells is summarized in Table 3-2. The axonal outgrowth of the cells seeded on the free-standing scaffolds is comparable to the axonal outgrowth of the cells seeded on scaffolds spun on a flat substrate. It can be concluded from the results here that the fiber scaffolds can help axonal outgrowth in vitro, and axons will follow the aligned fibers that make up a growth-permissive bridge across the glial scar tissue. Aligned fiber scaffolds have been shown to be successful in aiding axonal outgrowth. This raises the question as to the role of adhesion of the cells to the fibers in the success of the scaffolds in aiding axonal outgrowth. The PLLA appears to allow enough adhesion 
to keep the cells attached to the scaffolds but not such strong adhesion that the axonal outgrowth is impeded. These results also help model the behavior of cells and the interactions with free-standing fiber scaffolds in vitro. The next step is to improve upon the previous results and move closer to clinical use of these fiber scaffolds for SCI and neural tissue repair. It is thought that using a more complex, coaxial structure may provide more options for aiding axonal outgrowth, such as nerve growth factor (NGF) release and fibers with a conductive core. These structures are being explored and examined. 


\title{
4 Formation of Aligned Core/Sheath Microfiber Scaffolds
}

\author{
with a poly-L-lactic acid (PLLA) Sheath and a Conductive
}

poly(3,4-ethylenedioxythiophene) (PEDOT) Core $^{3}$

\subsection{Abstract}

Electrospinning of coaxial fibers to create core-sheath fiber structures to serve as multi-functional neural tissue scaffolds has been explored. A biocompatible polymer sheath combined with a core to provide additional growth cues and/or stimuli is the goal of this study. Here, the core material was the conductive polymer poly $(3,4-$ ethyelenedixoythiophene): poly(styrene sulfonate) (PEDOT:PSS) in oleic acid with a sheath of poly-L-lactic acid (PLLA). This created coaxial fibers with a conductive core and an insulating sheath to simulate an artificial axon. Several coaxial nozzle geometries were tested and the effect of operating and spinning solution parameters on fiber morphology examined. The most successful tests were with a nozzle using two different sized spinning needles. Coaxial fibers were spun using different spinning needle combinations, e.g. $22 \mathrm{G} / 16 \mathrm{G}$ and $22 \mathrm{G} / 17 \mathrm{G}$ (inner/outer). Key parameters in successfully spinning coaxial fibers included outer solution viscosity, inner/outer flow rate, field strength, and core solvent. The resulting fibers were characterized using fluorescence

\footnotetext{
${ }^{3}$ The material within this chapter will be submitted for review to the journal "Materials Science and Engineering C."

Citation:

Martin, Rachel, Wendling, Marie, Radke, Qian, Zichen, Dan, Zhao, Feng and Mullins, Michael.

"Formation of Aligned Core/Sheath Microfiber Scaffolds with a poly-L-lactic acid (PLLA) Sheath and a Conductive poly(3,4-ethylenedioxythiophene) (PEDOT) Core."
} 
optical microscopy and FE-SEM to confirm the coaxial structure and the conductive nature of the core. Fiber scaffolds were then characterized for fiber diameter, alignment and density. FTIR confirmed minimal mixing between the core and the sheath materials. Mechanical characterization was performed on two different diameter fibers $(6.1 \pm 2.4 \mu \mathrm{m}$ and $3.3 \pm 0.9 \mu \mathrm{m}$ ) using nanoindentation and the results compared to a solid PLLA microfiber.

\subsection{Introduction}

Spinal cord injuries (SCIs) continue to be among the most debilitating injuries due to the lack of regeneration capabilities of damaged neural tissue ${ }^{1}$. The development of a glial scar at the site of injury provides a hindrance to the repair, regrowth and reconnection of severed axons. Research to overcome this barrier has focused on the creation of an implantable scaffold that would act as a bridge across the glial scar and provide guidance for axonal regrowth ${ }^{22}$.

The technique of electrospinning to produce fiber scaffolds for axonal regeneration has been explored ${ }^{9,20,33,34}$. Polymer fiber scaffolds developed using poly-Llactic acid (PLLA) ${ }^{20,27,33,34,52,77}$, poly-caprolactone (PCL) ${ }^{31,39,40,49,54,62}$, poly(lactide-coglycolide) (PLG) ${ }^{106,107}$, poly(L-lactic acid) -co-caprolactone (PLL-CL) ${ }^{7,59}$, and silk fibroin ${ }^{55}$ have been studied and show promise in aiding axonal outgrowth in vitro. Studies that have been performed in in vivo models have not shown the same success as in vitro models. An injury model performed in vivo in rats with severed spinal cords using implanted conduits made of aligned PLLA fibers showed extensive regrowth of the 
transected spinal cord but did not show significant restoration of hind limb motor function $^{20,100}$.

Alternative approaches to electrospun fiber scaffolds are needed to improve their efficacy in vivo and improve the restoration of motor function. Approaches include spinning composite scaffolds with biopolymers such as collagen ${ }^{7}, \operatorname{laminin}^{27}$, or hyaluronic acid ${ }^{54}$ to improve biocompatibility and cell adhesion. Fiber scaffolds have also been developed with nerve growth factor (NGF) incorporated into the fibers to improve axonal outgrowth ${ }^{37,44}$. In addition to the use of biochemical agents to provide external stimuli during axonal regeneration during cell studies, electrical stimulation can be used to provide an additional source of stimulation for axonal outgrowth. Electrical stimulation has also been shown to enhance axonal outgrowth both in vitro and in vivo ${ }^{72,76,77,79}$.

Coaxial electrospinning is a way to create microfiber scaffolds with a core-sheath structure. This can be advantageous in repairing neural tissue, since more than one axonal growth stimuli can be incorporated, creating a multifunctional tissue scaffold. Encapsulating NGF or other drugs in the core of a coaxial fiber can help limit burst release and keep drugs active in vivo longer ${ }^{66,68,69}$. One potential application for coaxial fiber scaffolds is the use of conductive polymers. Electrical stimulation has been proven to aid in axonal outgrowth when the stimulation is applied along non-conductive fibers ${ }^{77}$. By using conductive polymers within the fiber scaffolds, it should be possible to create stronger local electrical fields near the surface of the fiber scaffold where the cells are in contact. When conductive polymers form the core of a coaxial fiber, pores or defects in the insulating sheath could allow for direct interaction between the axons and the 
conductive fibers when electrical activity occurs. One conductive polymer that has been studied is poly(3,4-ethylenedioxythiophene) (PEDOT) ${ }^{108-110}$. PEDOT is highly conductive $^{89}$ and has been studied for biological toxicity ${ }^{95}$. Poly(styrene sulfonate) (PSS) is often used as a counter-ion with PEDOT and improves solubility. In this present study, coaxial fiber scaffolds with an insulating, PLLA sheath and a conductive, PEDOT:PSS core were produced using coaxial electrospinning methods. The PLLA sheath provides a surface which has been proven to be attractive for axonal growth and adhesion; however, this study is the first to demonstrate the formation of a conductive core using a PEDOT:PSS suspension in aligned core-sheath scaffolds.

\subsection{Materials and Methods}

\subsubsection{Materials}

Poly-L-lactic acid (PLLA) was obtained from NatureWorks LLC and used as received. Chloroform was ordered from Sigma Aldrich and was used as the solvent for the PLLA sheath of the fibers. Oleic acid was ordered from Sigma Aldrich and used as the medium for the PEDOT suspension. Two different sources of PEDOT were used. PEDOT nanoparticles with DBSA as the counter-ion were ordered from Sigma Aldrich. 3,4-ethylenedioxythiophene (EDOT) was ordered from Alfa Aesar and used as received. Styrene sulfonic acid and sodium sulfate were ordered from Sigma Aldrich and used as received.

\subsubsection{PEDOT Synthesis}

Poly(3,4-ethylenedioxythiophene) with poly(styrene sulfonate) was synthesized from 3,4-ethylenedioxthyiophene (EDOT) (Alfa Aesar) monomer and poly(styrene 
sulfonic acid) (Sigma Aldrich) $(\mathrm{n}=10,000)$. The procedure used was an oxidative polymerization reaction with sodium sulfate as the oxidizing agent. A 1:1:2 molar ratio of EDOT: styrene sulfonic acid: sodium sulfate was used (1.4252g of EDOT, 1.8371g of poly(styrene sulfonic acid) and $2.3848 \mathrm{~g}$ of sodium sulfate). The EDOT monomer was mixed with $25 \mathrm{~mL}$ of distilled water prior to being added to the reaction solution. The poly(styrene sulfonic acid) was also mixed with $25 \mathrm{~mL}$ of distilled water before being added to the reaction solution. The sodium sulfate was mixed with $25 \mathrm{~mL}$ of distilled water before being added to the reaction solution. All three components were added to $25 \mathrm{~mL}$ of distilled water that was in a large beaker for a total volume of $100 \mathrm{~mL}$ of reaction solution. The concentration of each reactant in solution was $0.1 \mathrm{M}$. The solution as placed on a temperature-controlled heat plate at a constant temperature of $25^{\circ} \mathrm{C}$. The beaker covered with Parafilm ${ }^{\circledR}$ and left to complete the reaction overnight.

A color change was observed in the reaction solution overtime. The solution started as a pale-yellow color, which is the color of the EDOT monomer solution. At 30 minutes the solution turned a murky green color. At one hour, the solution turned a cloudy blue, and at 90 minutes it turned a very dark blue, almost black. After this, no color change was observed. A dark solid precipitate collected at the bottom of the beaker. After the reaction was complete, the product particles were collected using a fine filter and rinsed thoroughly with distilled water to remove any remaining ions. After drying, the product was a dark blue solid (Figure 4-1) with a total mass of $1.264 \mathrm{~g}$. The PEDOT:PSS product was analyzed using ATR-FTIR spectroscopy using a Perkin Elmer Spectrum One spectrometer to confirm the composition. 


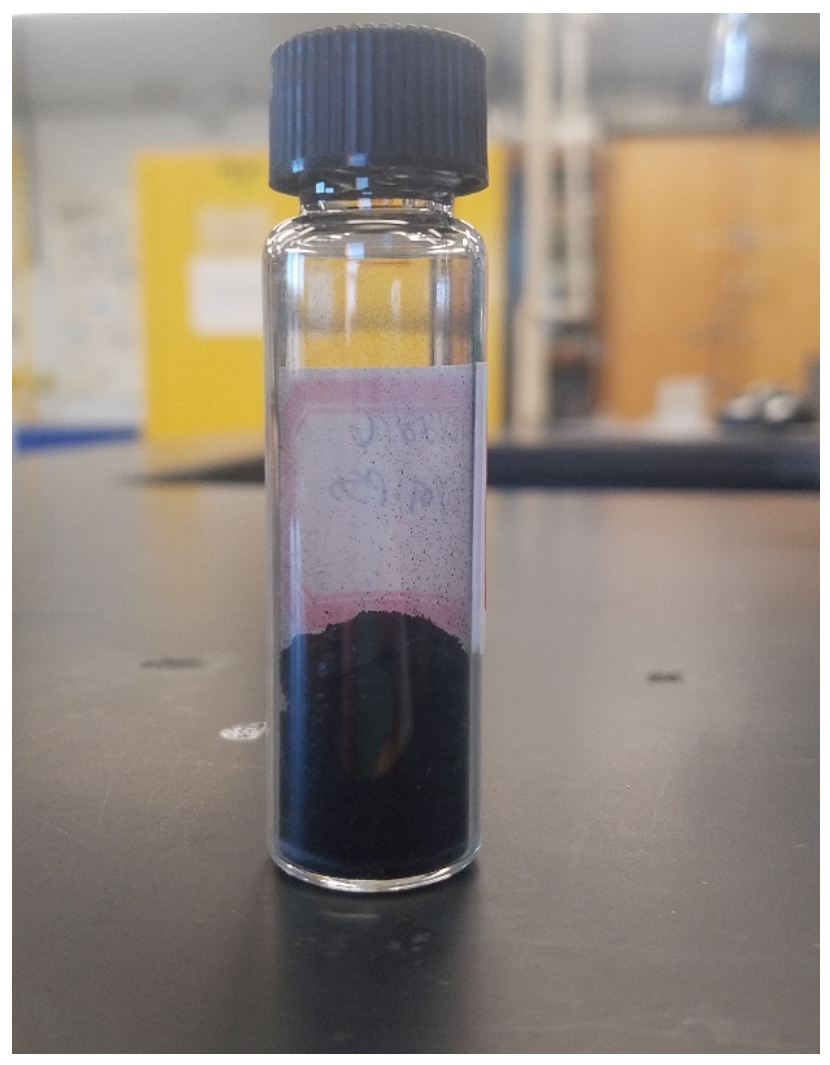

Figure 4-1: Synthesized PEDOT:PSS polymer after 24 hours of polymerization, double filtration and overnight drying.

\subsubsection{Coaxial Electrospinning Apparatus Design}

Coaxial electrospinning uses a conventional set-up as described previously with some modifications ${ }^{105}$. Previously, various nozzles were designed that would allow for electrospinning fibers with a core-sheath structure ${ }^{111}$. In the modified apparatus, two separate solutions (one for core solution, one for sheath solution) on separate syringe pumps are delivered to the coaxial nozzle via separate ports. The solutions are kept separate until they meet the nozzle, where they come together as the solutions are spun to 
create fibers with a core-sheath structure. Both inner and outer solutions must be connected to ground and the power supply as both solutions must be charged (Figure 42). The nozzle has a side port for the outer solution and an inner port for the core solution. In all of the nozzle designs, a $22 \mathrm{G}$ needle $(0.413 \mathrm{~mm}$ i.d.) serves as the spinneret for the core solution. Two different nozzle designs were examined for delivery of the outer sheath solution. One design had a side port connected to an aperture on the outside of the $22 \mathrm{G}$ needle with a diameter sufficient to encapsulate the core (Figure 4-3) with a diameter of 0.2065 inches. This nozzle aperture design was made in both Teflon ${ }^{\circledR}$ and stainless steel, with the charge applied only to the inner needle. Both nozzles were tested, but the stainless steel nozzle tended to produce stray currents and static charges, which increased fiber variability and decreased alignment. While the Teflon ${ }^{\circledR}$ nozzle did not produce stray currents, alignment of the needle and aperture was difficult and higher variability in fiber diameters was observed. 

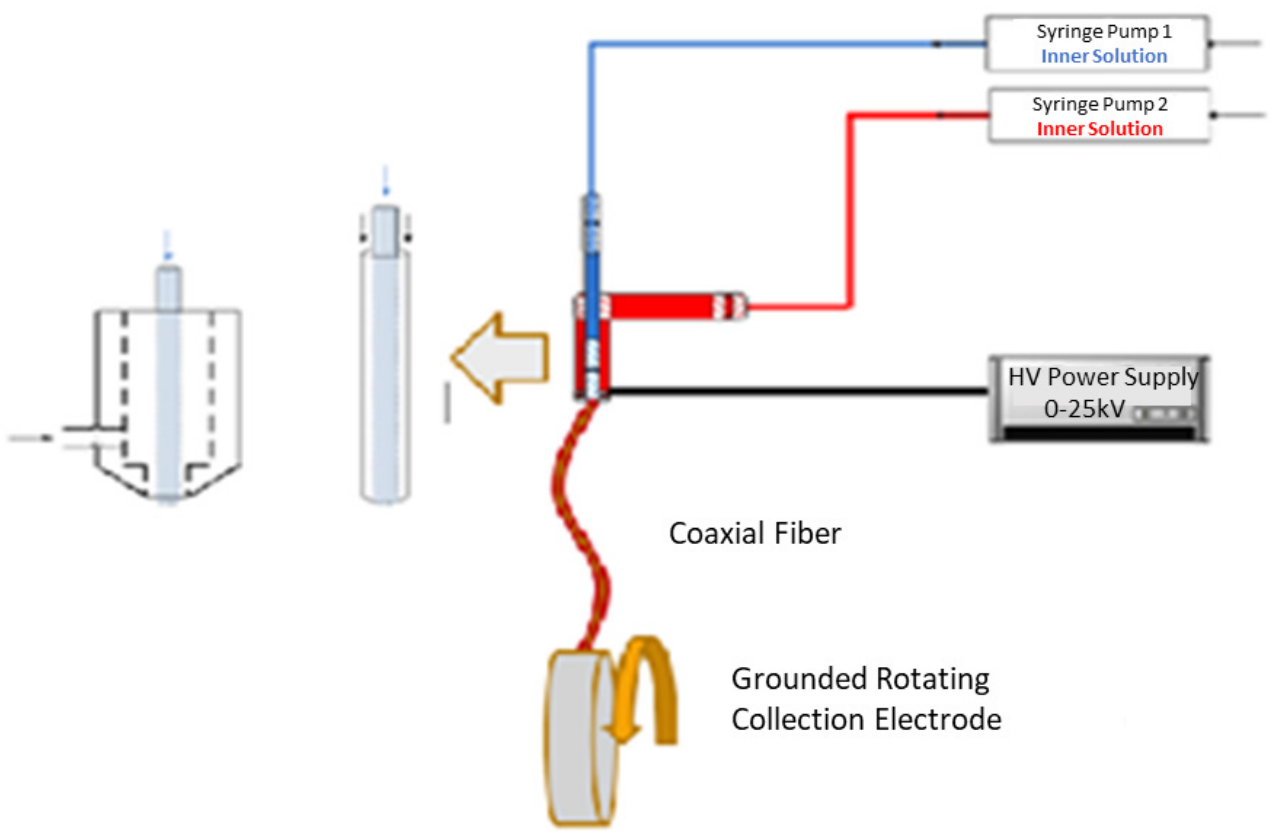

Figure 4-2: Schematic of coaxial electrospinning. The inner and outer solutions are kept separate until they reach the nozzle. The two different nozzle designs that were tested are shown. A coaxial strand is emitted from the Taylor's cone at the tip of the nozzle and collected on a rotating, grounded collector to achieve aligned, coaxial fiber scaffolds.

The second nozzle simply employed two concentric, blunt-tipped spinneret needles with sufficient space between the walls of the needles to allow free solution flow (Figure 4-3). The needles were mounted in a Teflon ${ }^{\circledR}$ housing with a side port to connect to the larger gauge needle and the inner $22 \mathrm{G}$ needle was directly connected to the syringe pump. This allowed for the power supply to be easily connected to both the inner and outer needles. A small piece of Tygon ${ }^{\circledR}$ tubing was placed over the outer needle to reduce stray currents. This design allows for the ability to spin fibers with different 
sheath diameters simply by using different needle diameters for the sheath solution. This design produced the most consistent spinning of coaxial fibers with uniform core and sheath diameters. Fibers spun using a 22G/16G spinning needle pair $(16 \mathrm{G}=1.194 \mathrm{~mm}$ i.d.) had a large diameter, whereas those spun with a $22 \mathrm{G} / 17 \mathrm{G}$ spinning needle pair $(17 \mathrm{G}=1.067 \mathrm{~mm}$ i.d. $)$ produced a smaller diameter sheath with a similar sized core. All nozzle designs are shown in Figure 4-3.

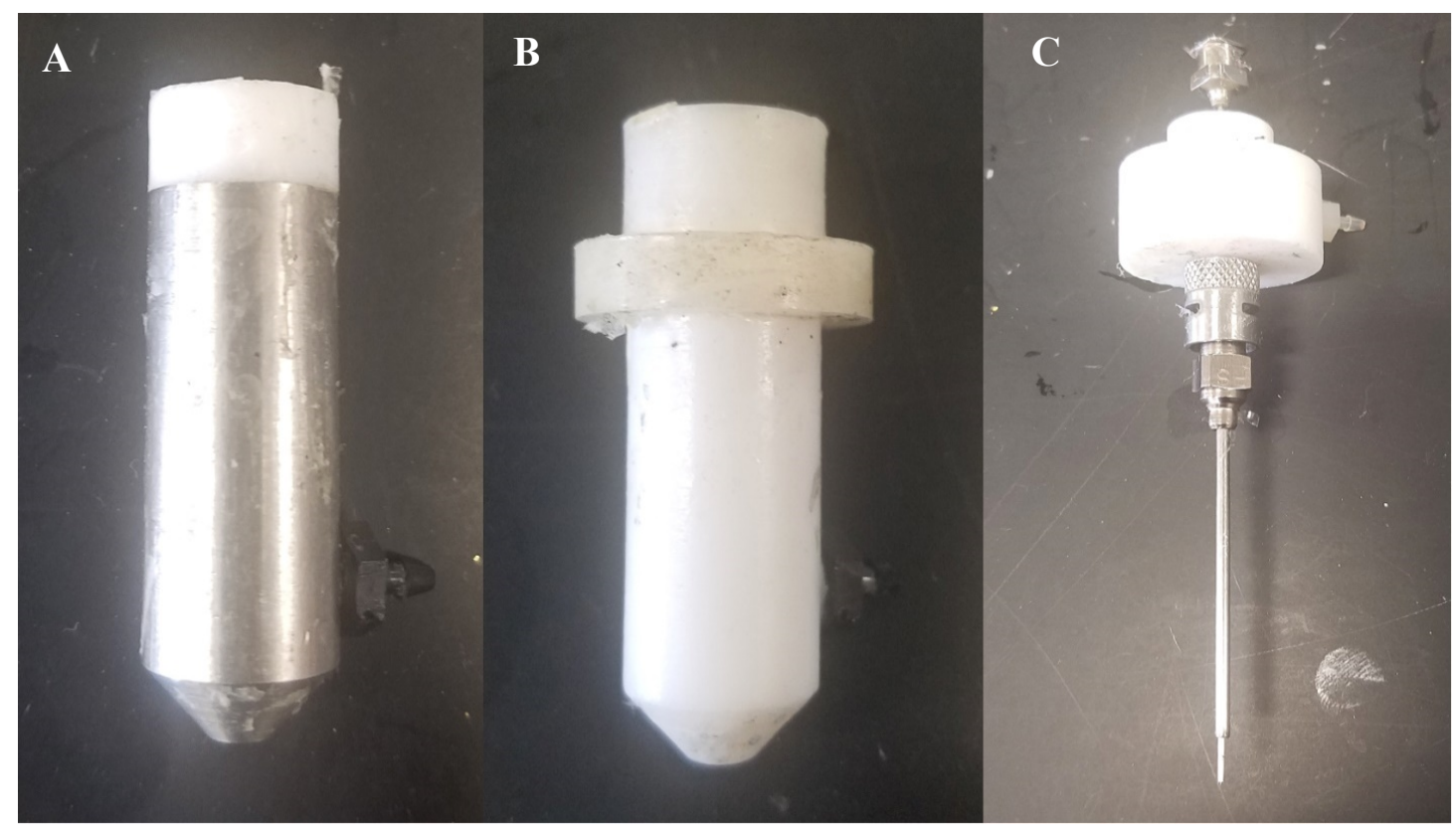

Figure 4-3: Different nozzle designs. (A) Stainless Steel nozzle (B) Teflon ${ }^{\circledR}$ nozzle (C) Dual needle nozzle 


\subsubsection{Testing of the Sheath Solution}

The outer solution was always PLLA for testing purposes since the goal was to spin fibers with a PLLA sheath. The inner solution was oleic acid after being determined as the best core material to use. Viscosity testing was done on the PLLA polymer solution to determine the optimum viscosity range for spinning consistent fiber scaffolds. For coaxial spinning, the viscosity of the inner solution is not as important because it is encapsulated in the sheath. However, the viscosity of the sheath solution, or the solution in single-stranded fiber electrospinning, is a vital parameter for achieving bead-free, consistent fibers and can also affect the diameter of the fibers. To determine the ideal range of viscosity for the PLLA solutions used in this study, a viscosity test was performed with various concentrations of PLLA solution in chloroform, and a viscosity profile plotted. The various solutions were also electrospun and imaged, and the images examined for fiber morphology and formation. A $10 \mathrm{wt} \%$ stock solution of PLLA was made to be diluted to use for the viscosity profile. $4.1427 \mathrm{~g}$ of PLLA were dissolved in $25 \mathrm{~mL}$ of chloroform to make a $10 \mathrm{wt} \%$ solution. The solution was placed on a combination stir/heat plate for approximately one hour until all of the PLLA was dissolved. Solutions of concentration $2 \mathrm{wt} \%, 4 \mathrm{wt} \%$ and $8 \mathrm{wt} \%$ were made from the $10 \mathrm{wt} \%$ solution, $10 \mathrm{~mL}$ each. The viscosity of $6 \mathrm{wt} \%$ solution was tested after making solution for one of the various spinning runs that were performed with that concentration. An AND SV-10 Vibro Viscometer was used to perform the viscosity testing. To measure the viscosity, $10 \mathrm{~mL}$ of each solution were placed in the supplied sample cups for the 
viscometer. The paddle-probes for the viscometer were then dropped into the solutions and the viscosity recorded from the read-out. After the viscosities were measured, the solutions were electrospun to examine the ability to spin the solutions and the morphology of the fibers formed.

\subsubsection{Preparation of the Core Solution/Suspension}

The PLLA polymer was always used as the sheath material to be consistent with our previous studies ${ }^{34,105}$. A variety of polymers were tested as suitable core materials. The first core system tested was poly-vinyl alcohol (PVA) in an 80/20 mixture of distilled water/ethanol. Multiple spinning tests were performed at different flow rate combinations and different voltages, but the solution was highly conductive and consistent coaxial fibers were not achieved. The next system tested was polypyrrole (PPY) suspended in distilled water. The polymer PPY is known be conductive and has been studied for use in creating conductive electrospun fibers ${ }^{88}$. However, the PPY disperses poorly in water even with surfactants, causing clumping and clogging of the nozzle. A similar approach was used with PEDOT:DBSA nanoparticles dispersed in water from Sigma Aldrich. These particles dispersed well and did not clog the needle, but the conductivity of the water was higher than desired. The final system tested was oleic acid as the medium in the core. The conductivity of the solution was not an issue, the viscosity is higher than water, and after adjusting the parameters, consistent coaxial fibers were obtained.

Once the best flow rate ratio was determined, a PEDOT suspension was added to the oleic acid in the core solution. Two different sources of PEDOT were used for testing, with each source utilizing a different counter-ion. One source of PEDOT was PEDOT 
nanoparticles from Sigma Aldrich that used dodecylbenzene sulfonic acid (DBSA) as a counter-ion. The other PEDOT that was used was polymerized in the lab from 3,4ethylenedioxythiophene (EDOT) with polystyrene sulfonate (PSS) as the counter-ion. Both were used to determine the effect of the different counter-ions on the conductivity and the biocompatibility of the fibers. For the PEDOT:PSS polymer, first a suspension of $2 \mathrm{wt} \%$ polymer in $10 \mathrm{~mL}$ of distilled water was made, since PEDOT:PSS is not soluble in oleic acid, and tends to form clumps that then clog the needle during electrospinning. By making a suspension of the polymer in water first, the particles form a finer suspension that clog the needle less frequently and the solution is easier to spin. The $2 \mathrm{wt} \%$ PEDOT:PSS suspension was then added to $10 \mathrm{~mL}$ of oleic acid to make a $15 \mathrm{wt} \%$ PEDOT:PSS: $\mathrm{H}_{2} \mathrm{O}$ in oleic acid suspension. The PEDOT:DBSA nanoparticles were already dispersed water, so the nanoparticle solution was added to $10 \mathrm{~mL}$ of oleic acid to also make a $15 \mathrm{wt} \%$ suspension.

\subsubsection{Coaxial Electrospinning Procedure}

Systematic testing of the electrospinning parameters was performed to determine

the best parameters to use for consistent coaxial fiber formation. Key parameters included the sheath solution viscosity, core medium for inner suspension, inner/outer flow rate ratios, and electric field strength. One of the most important parameters was found to be the ratio of the flow rates between the inner and outer solutions. To determine how the inner and outer flow rates affect the quality of the fibers, inner and outer flow rates were changed, fibers spun, and the samples imaged. The flow rates tested were $0.5 / 1.0 \mathrm{~mL} / \mathrm{hr}$, $1.0 / 1.0 \mathrm{~mL} / \mathrm{hr}, 0.8 / 1.2 \mathrm{~mL} / \mathrm{hr}$, and $0.5 / 1.5 \mathrm{~mL} / \mathrm{hr}$ (inner/outer). It was determined that the 
best ratio between the inner and outer flow rates for producing consistently coaxial fibers was $1: 3$; therefore, the flow rates chosen were $1.5 \mathrm{~mL} / \mathrm{hr}$ for the outer solution and $0.5 \mathrm{~mL} / \mathrm{hr}$ for the inner solution.

Process parameters such as field strength and wheel rotation rate were determined to spin aligned fiber mats of high quality. The voltage was set to $20 \mathrm{kV}$ and the needles were placed $10 \mathrm{~cm}$ above the wheel, giving an electric field strength of $2 \mathrm{kV} / \mathrm{cm}$. The wheel rotation rate used was $3000 \mathrm{rpm}$. These parameters were higher than those used for spinning pure PLLA fibers (17kV, 1.7KV/cm, 1250rpm).

Samples were spun at the previously determined flow rates for 10 minutes. During coaxial electrospinning, a larger, more elongated Taylor's cone was observed with the inner solution appearing inside the outer solution, and the cone encompassing both needles (Figure 4-4). The fiber samples were dried in a vacuum oven at $50^{\circ} \mathrm{C}$ for 30 minutes to remove residual solvent. The fiber samples were then imaged using fluorescence optical microscopy. 

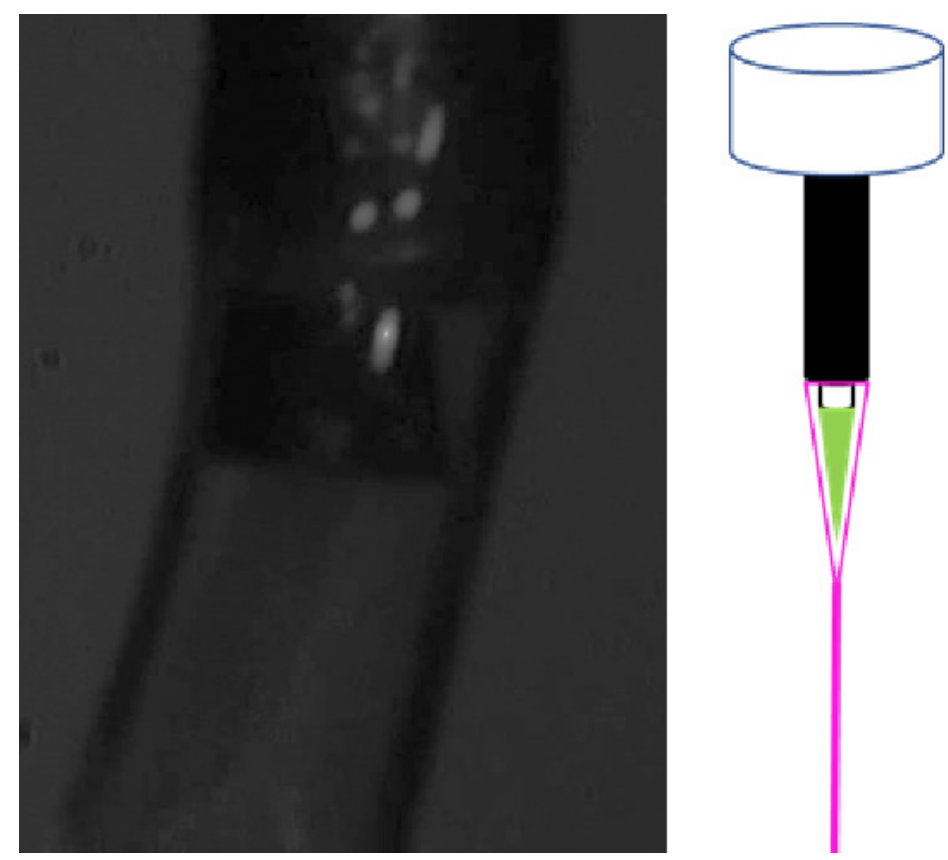

Figure 4-4: Coaxial Taylor's cone Left: During electrospinning, Right: Drawing. The inner solution can be seen as being fully encased in the outer solution.

\subsubsection{Fluorescence Optical Imaging and FTIR Fiber Analysis and}

\section{Characterization}

To analyze the fiber scaffolds and determine if coaxial fibers were achieved through electrospinning, fluorescence optical microscopy was done on the fibers. Images were taken of the fibers using an Olympus BX-15 optical microscope with a camera attachment and X-Cite 120 Fluorescence Illumination System. First a red image was taken of the fibers, then a green image. An overlay was created of the two images, and anywhere that yellow fibers appeared was determined to indicate that coaxial fibers were successfully electrospun. 
FTIR analysis was performed using a Perkin Elmer Spectrum One spectrometer. 50 scans were performed to obtain the spectra. Pure PLLA microfibers and coaxial PEDOT:PSS/PLLA (inner/outer) fibers were analyzed to determine the surface chemistry of the sheath.

\subsubsection{Field Emission Scanning Electron Microscopy Fiber Analysis and}

\section{Characterization}

To characterize the fiber scaffolds and determine if coaxial fibers were achieved through electrospinning, fluorescence optical microscopy was done on the fibers. Images were taken of the fibers using an Olympus BX-15 optical microscope with a camera attachment and X-Cite 120 Fluorescence Illumination System. For optical fluorescence analysis, the inner solution was dyed with a minute amount of the green fluorescent dye, coumarin 6. The outer solution was dyed with a minute amount of the red fluorescent dye, rhodamine B. For these conditions, the inner and outer solutions are completely immiscible. Using fluorescence microscopy, a green image is taken to show the coumarin dyed core and a red image is taken to show the rhodamine dyed sheath. When the images are overlaid in the computer, fibers that appear yellow indicate the formation of coaxial fibers. In some cases, the outer sheath shows an outline of red, but the core/sheath image is always yellow.

ATR-FTIR analysis was performed using a Perkin Elmer Spectrum One spectrometer. Pure PLLA microfibers and coaxial PEDOT:PSS/PLLA (inner/outer) fibers were analyzed to determine the surface chemistry of the fibers and determine if any mixing had occurred between the core and sheath. 


\subsubsection{Mechanical Testing of Coaxial Microfibers}

Mechanical properties of the fibers were measured and collected using a nanoindenter instrument with a Berkovich tip to calculate the modulus and hardness of the coaxial fibers. The Berkovich tip makes indentations in the surface of the sample, collecting load and displacement measurements that are then used to calculate the Young's modulus ${ }^{112}$. Pure PLLA fibers, coaxial fibers spun with a 22G/16G inner/outer spinning needle combination and coaxial fibers spun with a $22 \mathrm{G} / 17 \mathrm{G}$ inner/outer spinning needle combination were tested and the data collected. The indentation depth was $1500 \mathrm{~nm}$ to maintain consistent with previous testing ${ }^{112}$. Twenty points were taken per sample. The sample area was $5 \times 4$, and $50 \mu \mathrm{m}$ by $75 \mu \mathrm{m}$.

\subsection{Results}

\subsubsection{PEDOT:PSS Synthesis}

PEDOT:PSS polymer was successfully synthesized as determined by observation of the color and state change, FTIR analysis and electrical conductivity testing. The spectrum collected with ATR-FTIR is shown in Figure 4-5. The major characteristic peaks for PEDOT:PSS are seen in the spectrum ${ }^{109}$. Aromatic $\mathrm{C}=\mathrm{C}$ bending occurs around $1700 \mathrm{~cm}^{-1}$. This also accounts for the aromatic-like nature of the thiophene group. Peaks also associated with the C-S bonds are in the $600-700 \mathrm{~cm}^{-1}$ range. The peaks observed between 1200 and $1400 \mathrm{~cm}^{-1}$ are indicative of alkyl groups, and ester groups are observed between 1100 and $1200 \mathrm{~cm}^{-1}$. The change in color from the yellow monomer solution to the dark blue solid (Figure 1) was also a clear indication of successful polymerization of PEDOT:PSS. A conductivity cell with copper electrodes on either side of the cell was 
used to measure the conductivity of the polymer. The polymer was placed in the cell, the copper electrodes were placed on either side and a multimeter used to measure the resistance across the cell. The corresponding conductivity was measured to be $16.3 \mathrm{mS}$, or $54 \mathrm{mS} / \mathrm{cm}$. This is a little lower than usual; PEDOT conductivity is typically between 1 and $100 \mathrm{~S} / \mathrm{cm}^{89}$.

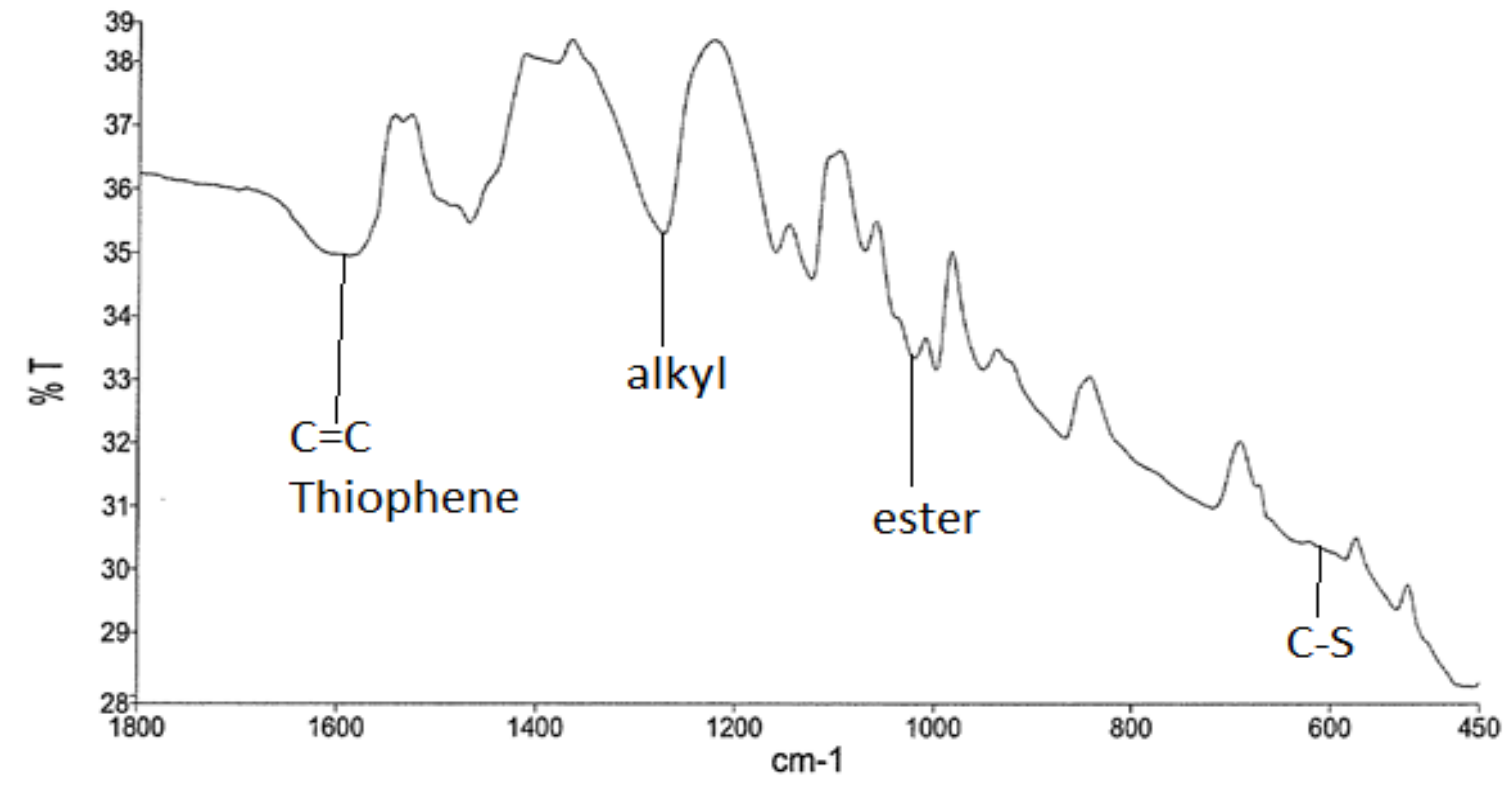

Figure 4-5: FTIR spectrum of synthesized PEDOT:PSS. Major characteristic peaks are labeled. 


\subsubsection{Viscosity and Flow Rate Analysis}

The viscosity testing that was performed found the ideal range of viscosity for the PLLA solution is $6-8 \mathrm{wt} \%$. Figure 4-6 shows the plotted viscosity data that was obtained, and Table 4-1 shows the corresponding data that was used to create the plot. Viscosity increases exponentially with increasing concentration until the viscosity is too high to allow for electrospinning to occur, providing a narrow range of ideal viscosity. The viscosity of the core solution of $15 \mathrm{wt} \%$ PEDOT:PSS: $\mathrm{H}_{2} \mathrm{O}$ in oleic acid was $27.4 \mathrm{cP}$.

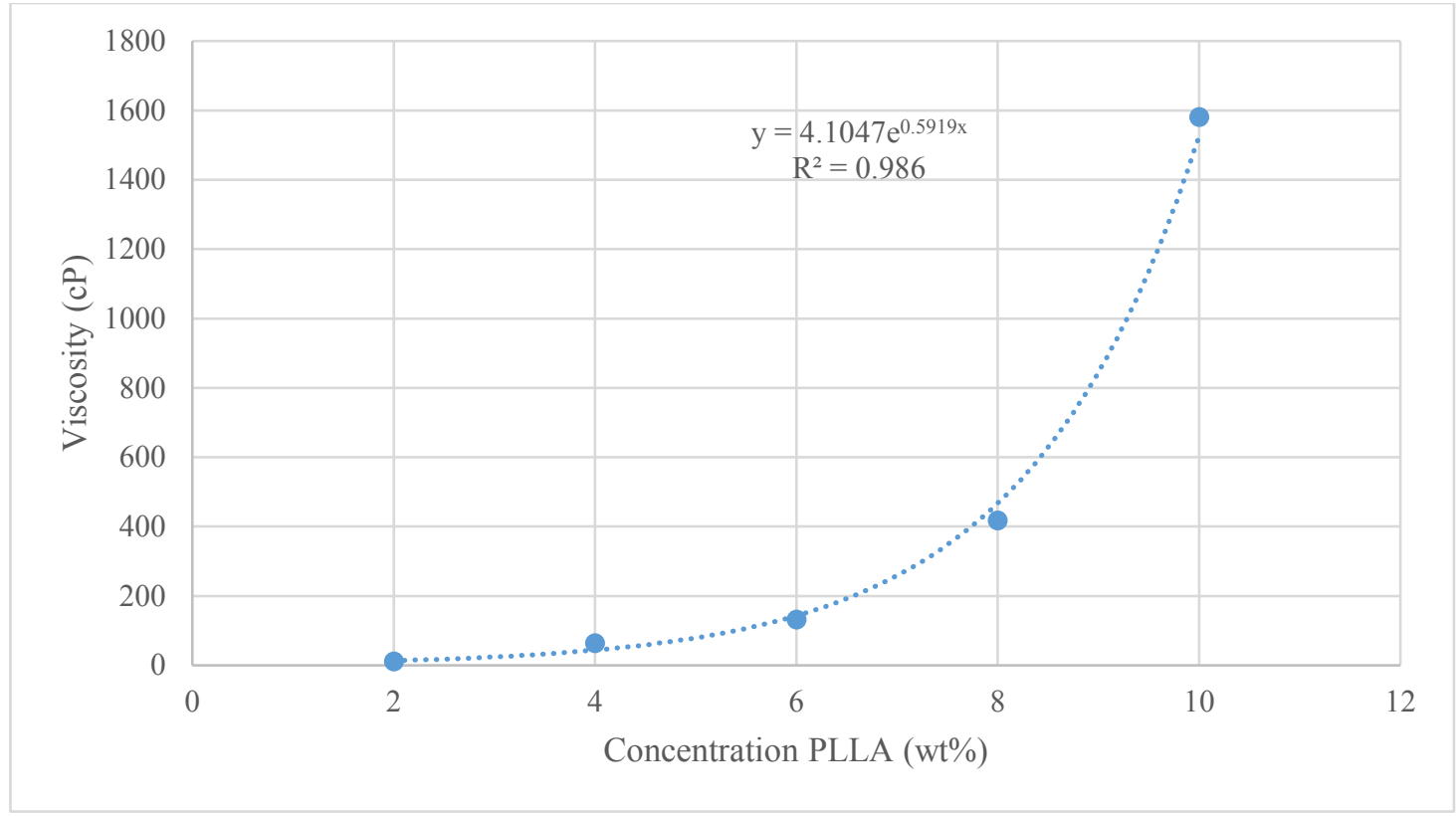

Figure 4-6: Viscosity profile of PLLA solutions from viscosity testing. 
Table 4-1: Viscosity data of PLLA solutions for viscosity profile.

\begin{tabular}{|c|c|}
\hline $\begin{array}{c}\text { Conc. PLLA } \\
(\mathbf{w t} . \%)\end{array}$ & Viscosity (cP) \\
\hline 2 & 10.9 \\
\hline 4 & 63.3 \\
\hline 6 & 132 \\
\hline 8 & 417 \\
\hline 10 & 1580 \\
\hline
\end{tabular}

Figure 4-7 shows the microscopy images obtained of the fibers spun from the solutions used in the viscosity testing. As can be observed, consistent, bead-free fibers were only obtained from the 6 and $8 \mathrm{wt} \%$ solutions. Figure 4-7A shows the imaged obtained from spinning the $2 \mathrm{wt} \%$ solution. The image indicates that electrospraying occurred rather than electrospinning, so droplets formed instead of fibers. Figure 4-7B shows the image obtained from spinning the $4 \mathrm{wt} \%$ solution. While fibers did form, there was a lot of beading on the fibers. Figures 4-7C and 4-7D show the images obtained from spinning the 6 and $8 \mathrm{wt} \%$ solutions, respectively. These concentrations formed consistent fibers. Figure 4-7E shows the image obtained from spinning the $10 \mathrm{wt} \%$ solution. While fibers did form, they were very thick and did not dry properly.

To determine the effect of the flow rate ratios on the formation of consistently coaxial fibers, electrospinning was done with several inner and outer flow rate ratios. The 
combinations used were $0.5 / 1.0 \mathrm{~mL} / \mathrm{hr}, 1.0 / 1.0 \mathrm{~mL} / \mathrm{hr}, 0.8 / 1.2 \mathrm{~mL} / \mathrm{hr}$, and $0.5 / 1.5 \mathrm{~mL} / \mathrm{hr}$ (inner/outer). Figure 4-8 shows the fibers created for each of these conditions. Figure 48 A shows the image obtained from the fibers spun with the $0.5 / 1 \mathrm{~mL} / \mathrm{hr}$ flow rate ratio. Some coaxial fibers are present, but not consistently. Figure 4-8B shows the image obtained from the fibers spun with the $1.0 / 1.0 \mathrm{~mL} / \mathrm{hr}$ flow rate ratio. No coaxial fibers were observed. Figure 4-8C shows the image obtained from the fibers spun with the $0.8 / 1.2 \mathrm{~mL} / \mathrm{hr}$ flow rate ratio. No coaxial fibers were observed. Figure $4-8 \mathrm{D}$ shows the image obtained from $0.5 / 1.5 \mathrm{~mL}$ flow rate ratio. Consistent coaxial fibers can be seen throughout the whole sample. 


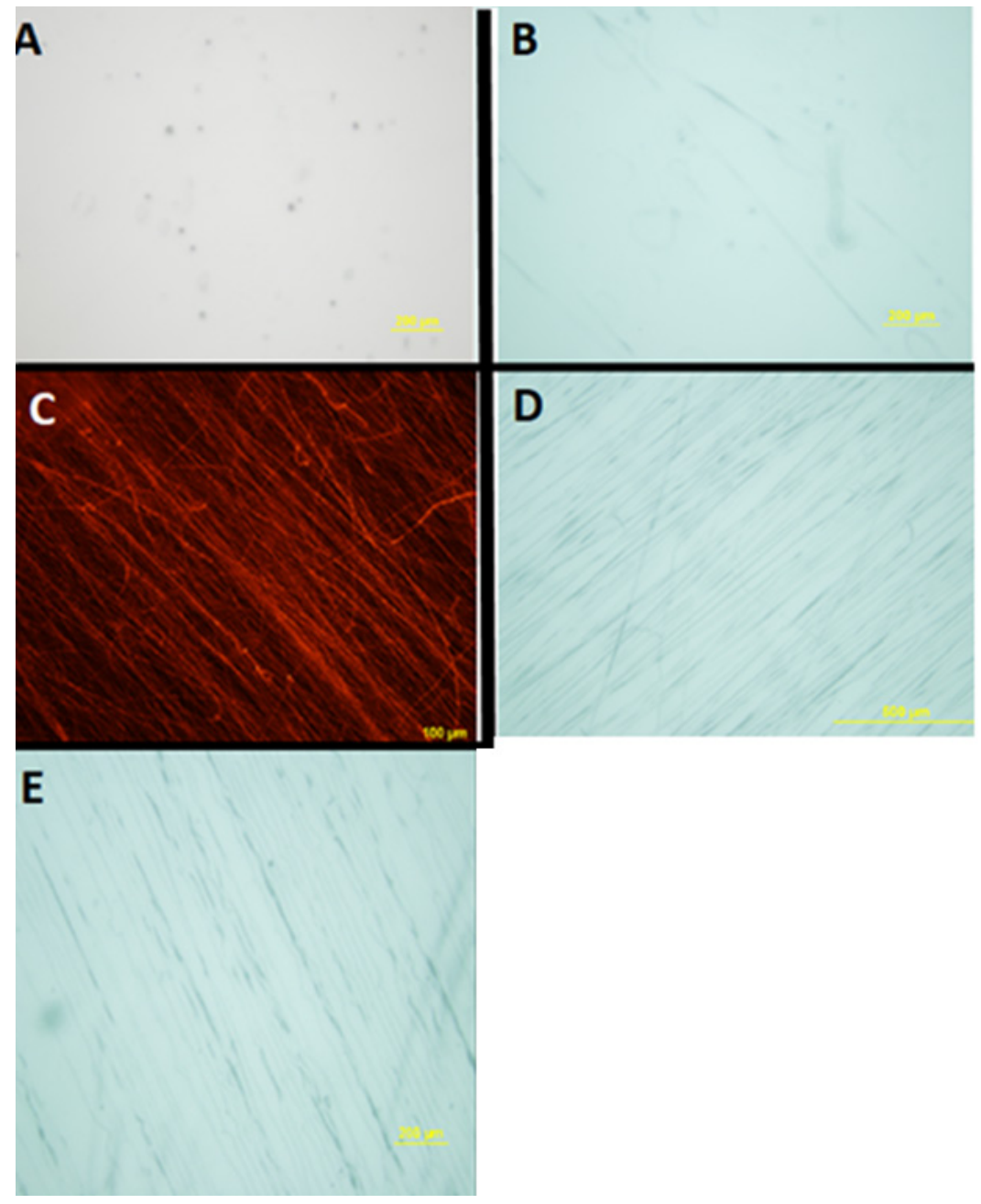

Figure 4-7: Images obtained from fibers spun during viscosity testing. (A) $2 w t \%$ solution (B) 4wt\% solution (C) 6wt\% solution (D) $8 w t \%$ solution (E) $10 w t \%$ solution 


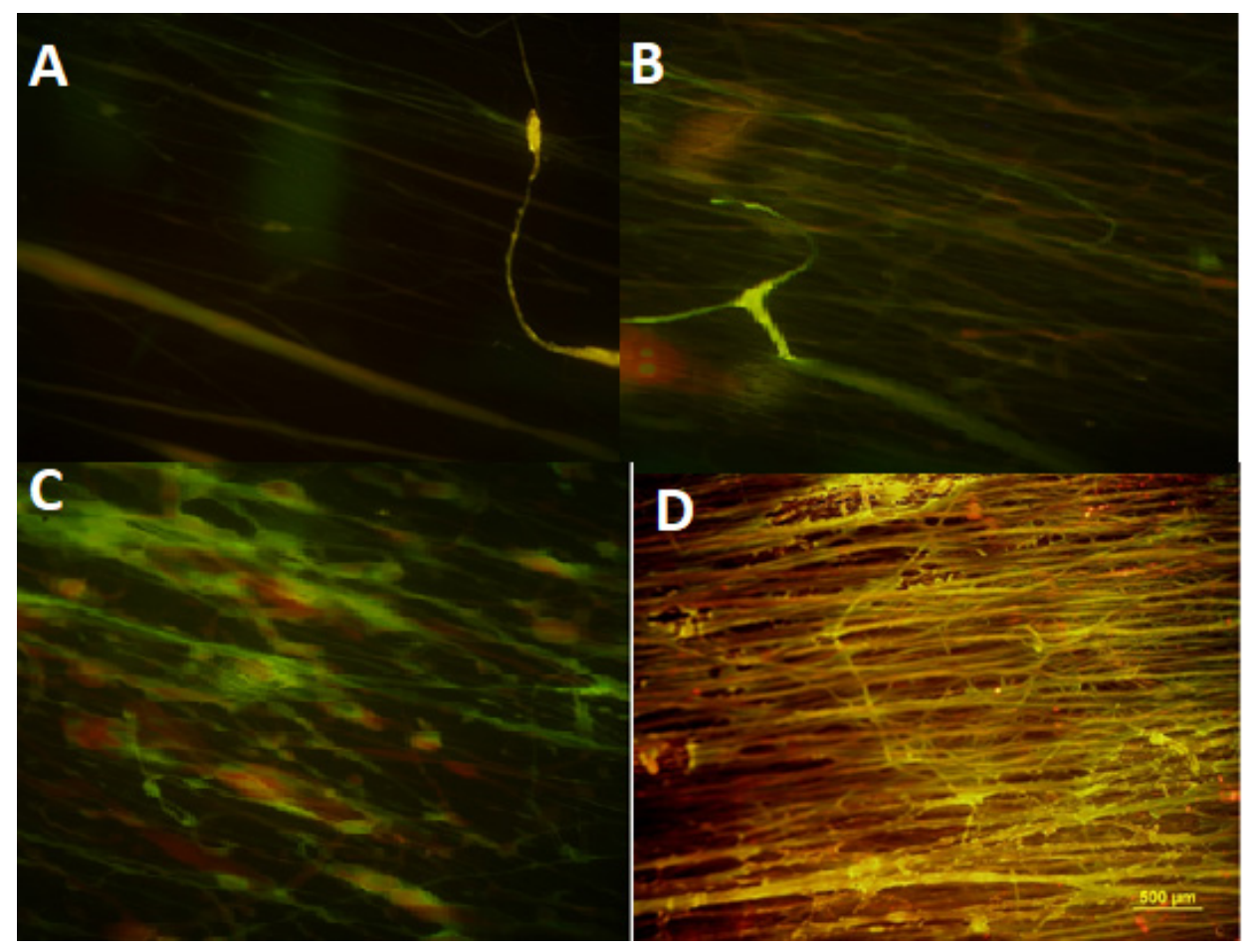

Figure 4-8: Flow rate analysis for coaxial fibers. (A) 0.5/1 (B) $1 / 1$ (C) 0.8/1.2 (D)

$$
0.5 / 1.5
$$

\subsubsection{Coaxial Fiber Characterization}

Fluorescence optical microscopy was used to determine the successful formation of core-sheath fibers. Red fluorescent dye in the sheath solution and green fluorescent dye in the core solution allowed for a red image to be taken and a green image to be taken using a digital microscope. When an overlay of the two images is created, any fibers that appear yellow indicate the formation of core-sheath fibers. Figure 4-9 shows the red, green and yellow overlay of a fiber sample collected with the $22 \mathrm{G} / 17 \mathrm{G}$ inner/outer needle set-up. 


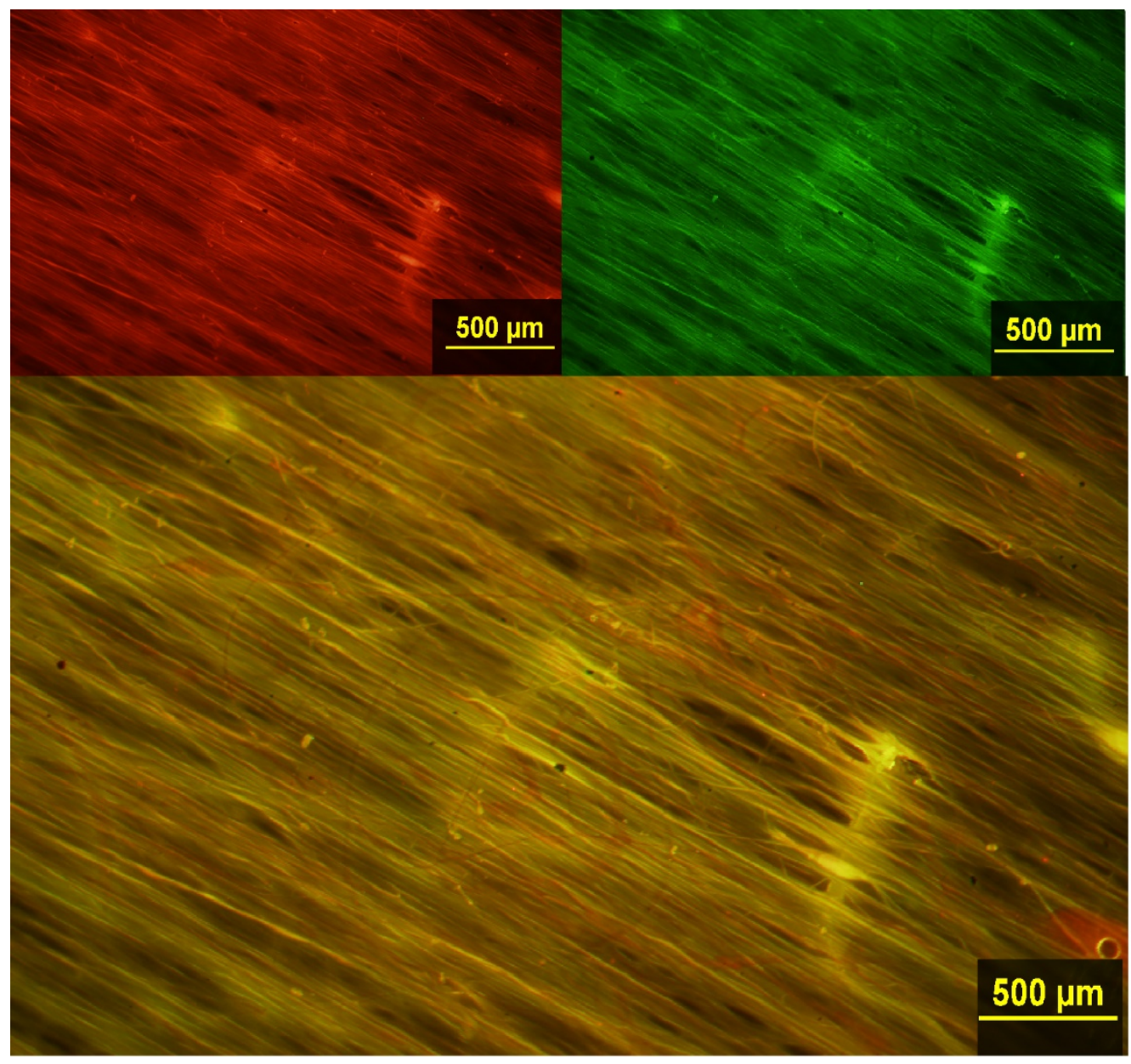

Figure 4-9: Fluorescent optical images of core-sheath electrospun fibers. The upper left photo shows the red image taken, the upper left photo the green image taken and the bottom photo the overlay of the two. The consistent yellow fibers across the sample indicate successful formation of core-sheath fibers. 
Field-Emission Scanning Electron Microscopy (FE-SEM) was performed on coaxial fiber samples obtained by freeze-fracturing of the aligned mats collected on flat coverslips. By examining the fracture surfaces of the fibers, the core-sheath morphology may be clearly seen (Figures 4-10 and 4-11). Under the high intensity electron beam, electrically insulating areas appear brighter than the surroundings due to the build-up of electrons. In this case, the PLLA sheath is insulating, causing the material to appear bright in the image. On the other hand, electrons can travel along conducting materials, and there is no brightening effect since the electrons do not collect on the surface. This can be seen in Figure 4-10, where the brightness of the PLLA sheath as compared to the PEDOT core indicates the formation of a fiber with a conductive core with an insulating sheath. In Figure 4-10, the sheath has a diameter of $6.7 \mu \mathrm{m}$, and the core has a diameter of $1.2 \mu \mathrm{m}$. The fiber in the image was spun using the $22 \mathrm{G} / 16 \mathrm{G}$ electrospinning needle setup, and using the PEDOT:DBSA nanoparticles. 
Lower detector (No charging contrast)

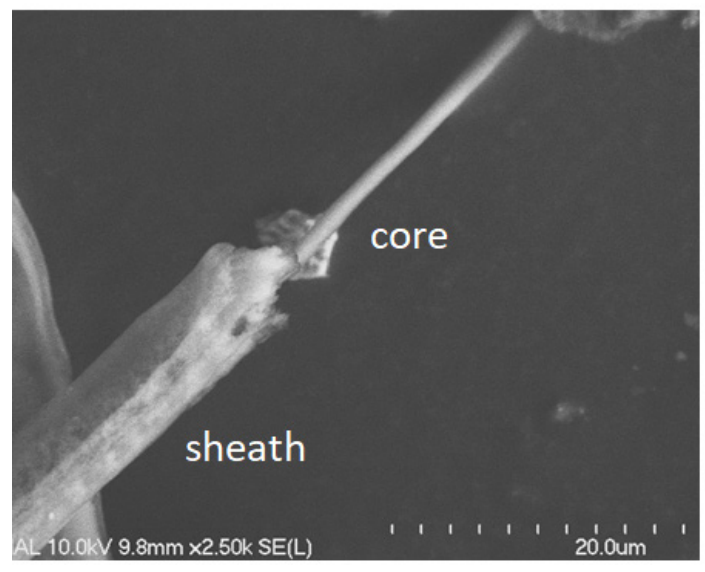

Upper detector (charging contrast)

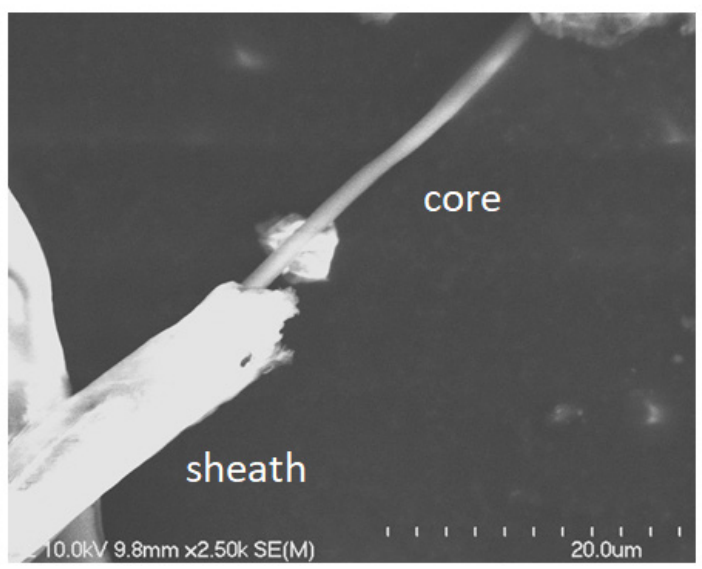

Figure 4-10: SEM image of core-sheath fiber comparing the lower detector image to the higher detector image. The brighter appearance of the sheath while the core has no change in appearance indicates the formation of core-sheath fibers with a conductive core and insulating sheath.

SEM analysis of the fibers spun with the $22 \mathrm{G} / 17 \mathrm{G}$ spinneret combination was also performed, and the core of those fibers also showed electrical properties. An example is shown in Figure 4-11. The core in this figure was spun with PEDOT:PSS. This image shows the sheath split down the center and the core in the middle. The sheath has a diameter of $4.8 \mu \mathrm{m}$ and the core has a diameter of $1.9 \mu \mathrm{m}$. 


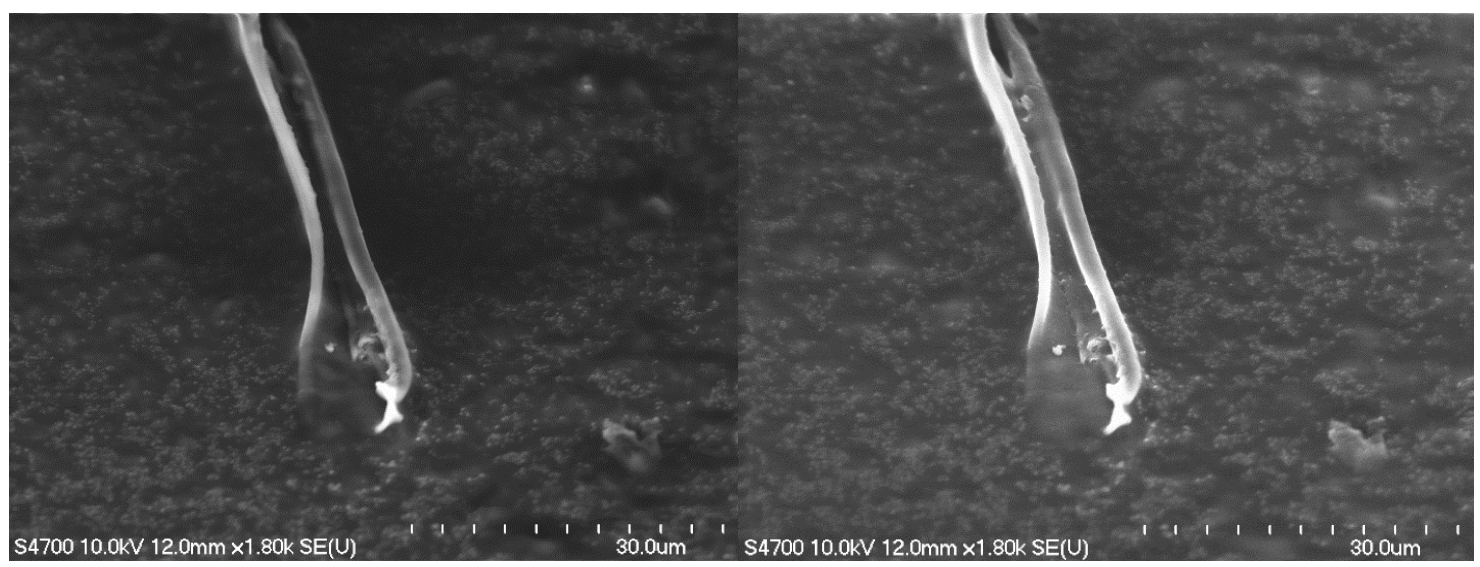

Figure 4-11: SEM of smaller diameter core-sheath fibers. The sheath is split down the middle, revealing the conductive core inside.

The diameter of the fibers was measured using Image ${ }^{\circledR}$ and the DiameterJ ( plug-in for the program. The diameter of pure PLLA fibers, coaxial fibers spun with a 22G/16G inner-outer spinning needle combination, and coaxial fibers spun with a $22 \mathrm{G} / 17 \mathrm{G}$ inner-outer spinning needle combination were measured and compared. The average diameter of the coaxial fibers spun with a $17 \mathrm{G}$ outer needle was $3.30 \pm 0.96 \mu \mathrm{m}$. The average diameter of the fibers spun with the $16 \mathrm{G}$ outer needle was $6.11 \pm 2.43 \mu \mathrm{m}$. The average diameter of PLLA single-walled fibers was $2.8 \pm 1.3 \mu \mathrm{m}$. Alignment analysis was also performed on the two different types of coaxial fibers. The smaller diameter coaxial fibers had a $\sigma=17.9^{\circ}\left(2 \sigma=39.4^{\circ}\right)$. The larger diameter coaxial fibers had a $\sigma=$ $10.8^{\circ}\left(2 \sigma=21.6^{\circ}\right)$. The pure PLLA fibers had an alignment of $\sigma=5.1^{\circ}\left(2 \sigma=10.2^{\circ}\right)$. These results are summarized in Table 4-2. Figure 4-12 shows optical images obtained comparing the smaller and larger fiber diameter coaxial fibers. While the $2 \sigma$ value for the smaller diameter coaxial fibers was quite large, further analysis showed most of the fibers, $70 \%$, were within $10^{\circ}$ of the angle of alignment. For the larger diameter fibers, 
$70 \%$ were within $8^{\circ}$ of the angle of alignment. This indicates that cross-fibers were present in both sets of samples, which greatly affected the alignment statistics of the fibers, and it may not reflect the general quality of alignment.

Table 4-2: Diameter, alignment and density data for different fiber samples

\begin{tabular}{|c|c|c|c|}
\hline $\begin{array}{c}\text { Sample } \\
\text { Conditions }\end{array}$ & $2 \sigma$ (degrees) & $\begin{array}{c}\text { Average Fiber } \\
\text { Diameter }(\mu \mathrm{m} \pm)\end{array}$ & $\begin{array}{c}\text { Average Fiber } \\
\text { Density(fibers } / \mathrm{mm} \pm \text { ) }\end{array}$ \\
\hline $\begin{array}{c}\text { single } \\
\text { walled fibers }\end{array}$ & $10.2^{\circ}$ & $2.8 \pm 1.3$ & $390 \pm 41$ \\
\hline $\begin{array}{c}\text { coaxial } \\
\text { fibers spun } \\
22 \mathrm{G}-17 \mathrm{G}\end{array}$ & $39.4^{\circ}$ & $3.3 \pm 0.9$ & $53 \pm 6.6$ \\
\hline $\begin{array}{l}\text { coaxial } \\
\text { fibers spun } \\
22-16 \mathrm{G}\end{array}$ & $21.6^{\circ}$ & $6.1 \pm 2.4$ & $28 \pm 4.3$ \\
\hline
\end{tabular}

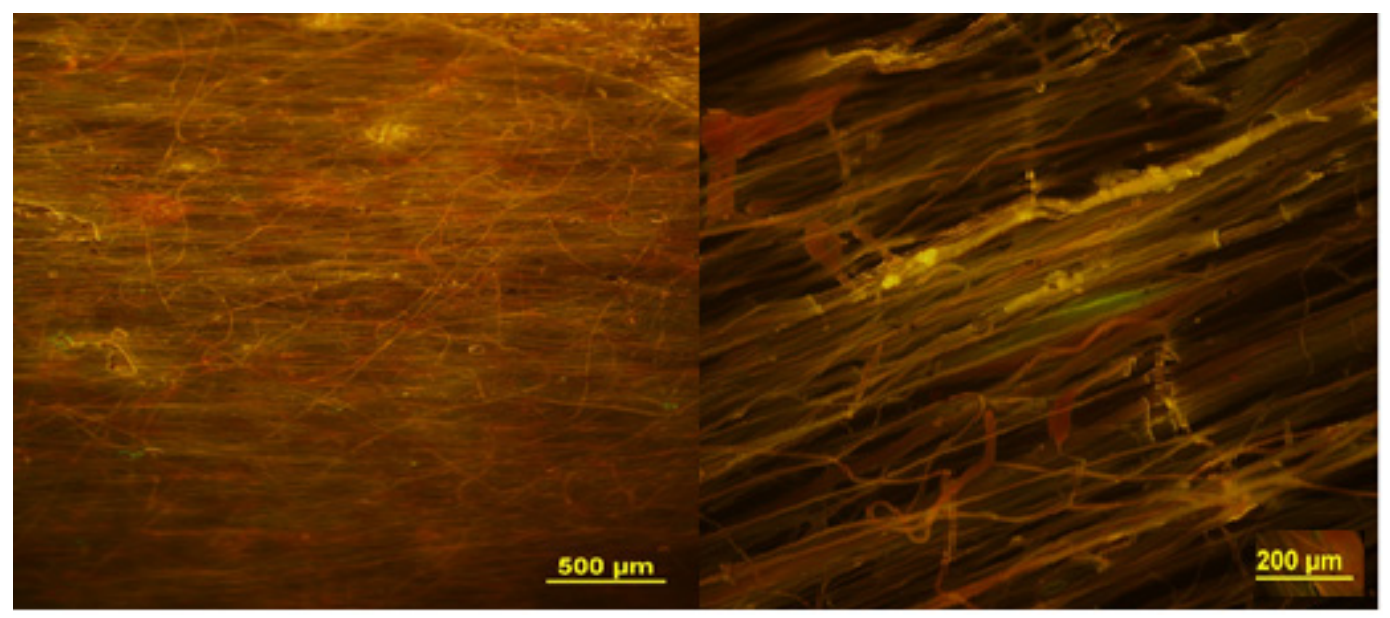

Figure 4-12: Fibers spun with different diameters. The left-hand figure spun with22$17 G$ system. Right hand spun with $22 G-16 G$ system. 


\subsubsection{Chemical and Mechanical Property Analysis}

ATR-FTIR analysis was performed on the surface of both pure PLLA fibers and coaxial core-sheath fibers to compare the surface chemistry between the two different types of fibers, and measure any mixing between the core and sheath materials. The spectra collected are shown in Figure 4-13. The structure along with the spectrum showing the peaks of PLLA is shown. The PLLA spectrum shows a peak between 1800 and $1600 \mathrm{~cm}^{-1}$, the characteristic ester peak of the PLLA structure. There are also methyl peaks around $1200 \mathrm{~cm}^{-1}$ and a C-C peak between 1200 and $1000 \mathrm{~cm}^{-1}$. The spectrum of the coaxial fiber surface only shows peaks identifying with PLLA and none of the characteristic PEDOT:PSS spectra. 

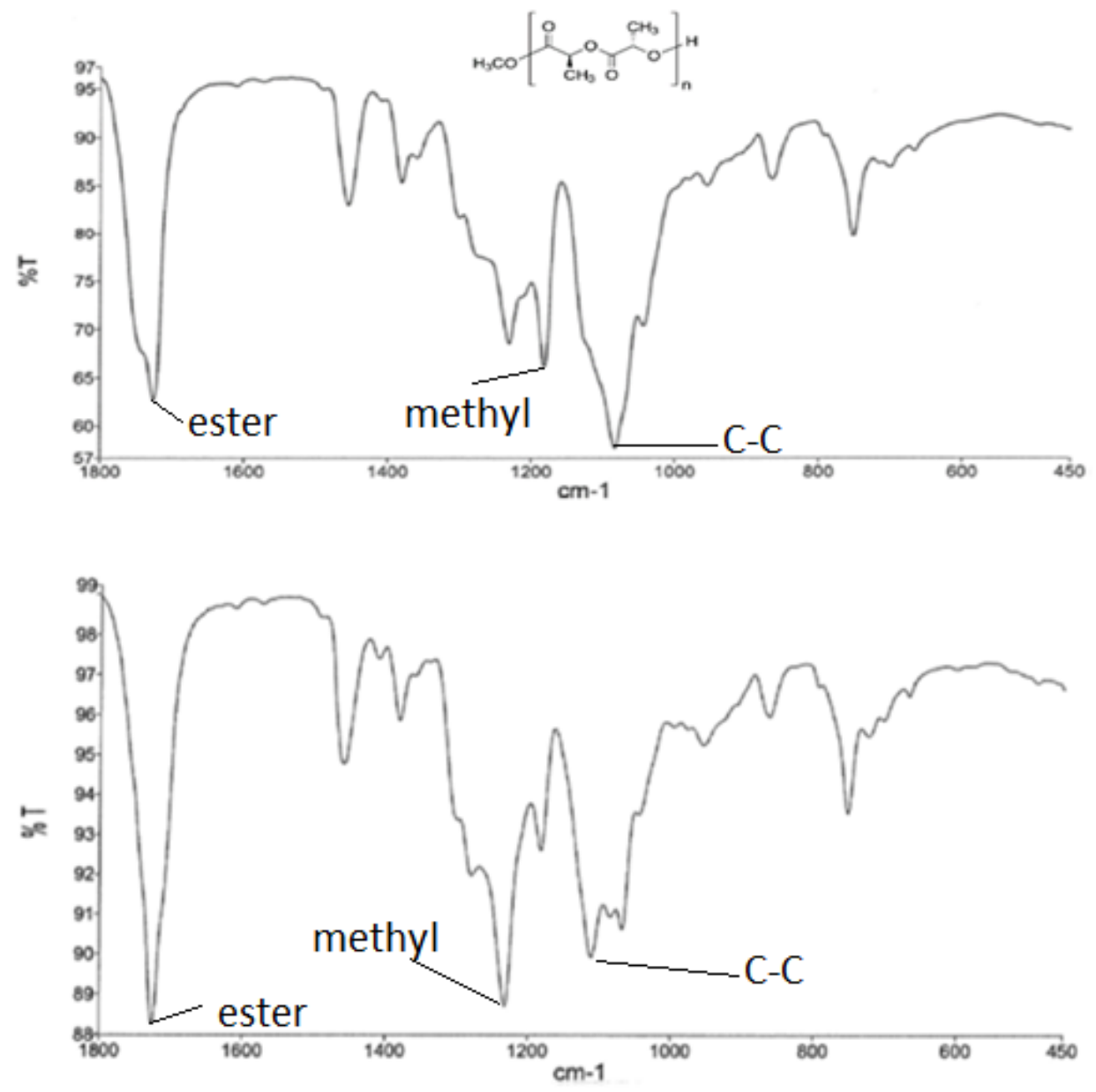

Figure 4-13: IR spectrum for (upper) PLLA fibers and (lower) coaxial fibers. Key characteristic peaks are labeled.

Mechanical information about the Young's modulus and the hardness of the fibers was collected using a nanoindenter with a Berkovich tip. The indentation depth was $1500 \mathrm{~nm}$. Due to the large diameters of the coaxial fibers $(>3 \mu \mathrm{m})$, it was determined that $1500 \mathrm{~nm}$ would be on the order of the sheath thickness, but would not go through the entire fiber sample. PLLA fiber data collected previously was collected at a depth of $1500 \mathrm{~nm}$, so this depth was consistent and can be directly compared. The results are 
summarized in Table 3 . The average modulus for the smaller core-sheath fibers was $0.7 \pm$ $0.4 \mathrm{GPa}$ and the average hardness was $0.03 \pm 0.03 \mathrm{GPa}$. The average modulus for larger core-sheath fibers was $0.16 \pm 0.13 \mathrm{GPa}$ and the average hardness was $0.022 \pm 0.01 \mathrm{GPa}$. This data may be compared to the values previously collected of $1.22 \pm 0.19 \mathrm{GPa}$ and 0.05 $\pm 0.006 \mathrm{GPa}$ of pure PLLA fibers ${ }^{112}$.

Table 4-3: Mechanical testing data for coaxial fibers

\begin{tabular}{|c|c|c|}
\hline Sample & Young's Modulus(GPa) & Hardness(GPa) \\
\hline small diameter fibers & $0.7 \pm 0.4$ & $0.03 \pm 0.03$ \\
\hline large diameter fibers & $0.16 \pm 0.13$ & $0.02 \pm 0.01$ \\
\hline PLLA $^{112}$ & $1.22 \pm 0.19^{112}$ & $0.05 \pm 0.006^{112}$ \\
\hline
\end{tabular}




\subsection{Discussion}

The results of the imaging and spectroscopic indicate the successful formation of aligned core-sheath microfiber scaffolds. The fluorescence optical imaging utilizing a red and green image overlay on samples spun with a PLLA sheath and PEDOT:PSS core indicate fibers that were coaxial in nature consistently showed a yellow overlay signature (Figure 9). The FE-SEM imaging confirms this by showing many instances where the core is separated from the sheath, either at the end or where the sheath had split down the middle. The surface charging observed in the FE-SEM images of the coaxial fibers indicates that the fibers have a conductive core with an insulating sheath (Figures 4-10 and 4-11). However, the conductivity of the core has not been quantitatively measured.

FTIR spectra shown in Figure 4-13 comparing the pure PLLA fiber and the coaxial fiber surface show no significant differences. The same peaks observed for PLLA fibers also appear in the spectrum for the coaxial fibers, with no additional peaks that might be expected for PEDOT:PSS. This leads us to conclude that only PLLA was present along the surface, with the core material fully encased in the sheath, indicating no splitting of the Taylor's cone into two separate fiber strands.

Fiber image analysis was performed on coaxial fiber scaffolds with different sheath diameters (Figure 4-12). The two systems studied were coaxial fibers spun with a $22 \mathrm{G} / 17 \mathrm{G}$ spinning needle combination and fibers spun with a $22 \mathrm{G} / 16 \mathrm{G}$ spinning needle combination. The larger coaxial fibers had an average fiber diameter of $6.1 \pm 2.4 \mu \mathrm{m}$, much larger than the diameter of pure PLLA fibers. The smaller coaxial fibers had an average fiber diameter of $3.3 \pm 0.9 \mu \mathrm{m}$. This diameter is much closer to the measured 
diameter of pure PLLA fibers, which was $2.8 \pm 1.3 \mu \mathrm{m}$. The diameter of the smaller coresheath fibers falls within the standard deviation of the PLLA fibers. However, the diameter of the larger core-sheath fibers fell outside this standard deviation. The density of the fibers was also analyzed. The scaffolds spun with fibers that had a smaller sheath diameter had a higher density than the scaffolds spun with fibers that had a larger sheath diameter (53 vs. 28 fibers $/ \mathrm{mm}$ ). This makes sense because the smaller diameter fibers can be more densely packed into a smaller area.

Analysis of the alignment of the coaxial fibers showed that the overall $2 \sigma$ value was quite large for the smaller diameter fibers, at $39.4^{\circ}$. However, when doing the analysis for the deviation of individual fibers from the axis of alignment, $70 \%$ of the fibers were within $10^{\circ}$. This indicates that cross-fibers developed on the sample during electrospinning, and these outlying fibers may be creating statistical artifacts. The same was true for the larger diameter coaxial fibers. The overall $2 \sigma$ was around $21^{\circ}$, but analysis of the individual fibers from the axis of alignment showed $70 \%$ were within $8^{\circ}$. This again indicates the presence of cross fibers but generally good alignment. Both sets of fibers had a lower degree of alignment than pure PLLA fibers, which had a $2 \sigma$ of about $10^{\circ}$. The difference is attributable to the higher rotation rate for the collection required (3000rpm vs $1250 \mathrm{rpm})$ by the higher polymer flow rates used for coaxial fibers $(2 \mathrm{~mL} / \mathrm{hr}$ vs $0.9 \mathrm{~mL} / \mathrm{hr}$ ). The higher wheel rotation rate required for coaxial fibers may also be due to the thicker strand pulled from the Taylor's cone for the coaxial fibers than for the PLLA fibers, requiring more force to pull them into aligned scaffolds. To improve the alignment may require collection at an even higher wheel rotation rate. The coaxial fibers 
are also exposed to a higher voltage than the pure PLLA fibers (20KV vs $17 \mathrm{KV})$, since more electrostatic force is required to overcome the surface tension in the dual droplet to distort the droplet into a coaxial Taylor's cone.

Viscosity testing showed a concentration range of the PLLA solution is between 6 and $8 \mathrm{wt} \%$ to create bead-free fibers (Figure 4-7). This indicates that the ideal range of viscosity is between 130 and 420cP (Table 4-1). The results of the viscosity profile indicated solutions with that low of a viscosity would case beaded fibers to form (Figure 4-7). However, the viscosity of the core oleic acid-PEDOT solution was around $27 \mathrm{cP}$. The viscosity of the inner solution did not have an effect on the morphology of the surface of the coaxial fibers. The inner solution can be of a lower viscosity because it becomes encapsulated in the sheath solution during the formation of the dual Taylor's cone, so the viscosity of the sheath solution becomes dominant. Thus, the coaxial nature of the fibers allows for a great deal of flexibility in the selection of the core solutions and allows for solvents to be spun that would not normally create polymer fibers alone.

Reproducible testing is dependent on the indenter tip being directly over the center of the fiber prior to indentation, and striking the fiber at an angle may cause skidding of the probe and produce skewed data. The Young's modulus was smaller for both sets of coaxial fibers than for pure PLLA. The modulus of the pure PLLA fiber was around $1 \mathrm{GPa}$, whereas the moduli for the smaller diameter and larger diameter coaxial fibers were $0.7 \mathrm{GPa}$ and $0.16 \mathrm{GPa}$, respectively. The thickness of the PLLA sheath around the core could affect the stiffness and allow the fiber to bend more easily under the indenter tip until the core was reached. The modulus for the larger diameter fibers was 
smaller than the modulus for the smaller diameter fibers. Again, the larger sheath diameter around the core would make the fibers more elastic (Table 3). Also, the indentation depth of $1500 \mathrm{~nm}$ indicates that the indenter was almost penetrating through the whole PLLA sheath, particularly for the smaller diameter fibers. The pure PLLA fibers are solid and would have less flex than coaxial fibers with a softer core. The surface hardness was similar for all the fibers, since the surface compositions are the same. Penetration of the sheath material at the maximum indentation depth could lead to inconsistent measurements, but was not apparent in our data.

\subsection{Conclusion}

In this study, an apparatus and its associated process parameters necessary to electrospin highly aligned, coaxial microfibers were studied. The key process and solution parameters were found to be the sheath solution viscosity, the core solution conductivity, the collection wheel rotation rate, and the ratio of the core-to-sheath flow rate. A highly aligned, novel core-sheath fiber structure with a conductive polymer core and an insulating sheath was successfully produced and characterized. The polymer sheath was composed of biocompatible poly-L-lactic acid (PLLA) studied previously as a neural tissue scaffold to aid axonal outgrowth ${ }^{34}$ Viscosity testing indicated a concentration for the PLLA polymer solution for the sheath of between 6 and $8 \mathrm{wt} \%$ would produce consistent, bead-free fibers. PEDOT:PSS particles $(<1 \mu \mathrm{m})$ were synthesized through oxidative polymerization of the 3,4-ethylenedioxythiophene (EDOT) monomer, and a conductive polymer core solution was prepared by using a poly $(3,4-$ ethylenedioxythiophene): poly(styrene sulfonate) (PEDOT:PSS) suspension. When water 
was used as the dispersing medium, the electrical conductivity of the suspension was too high to allow successful electrospinning. To reduce the conductivity, oleic acid was used as the suspension medium, which lowered the conductivity and allowed the successful formation of a conductive core with an insulating PLLA sheath. Coaxial fibers of different outer diameters were spun using two combinations of core/sheath needles (22G/16G inner-outer and 22G/17G inner-outer combinations). Testing of various flow rate ratios for the core and sheath solutions indicated that the ratio of core to sheath flow rate of $0.5 / 1.5 \mathrm{~mL} / \mathrm{hr}(1: 3)$ created the most consistently coaxial fibers for this system.

Fluorescence microscopy, FE-SEM imaging, and ATR-FTIR surface analysis all indicated successful formation of highly-aligned electrospun microfibers with a conductive, PEDOT:PSS core and an insulating PLLA sheath. Analysis of the surface charging from SEM images of the core/sheath fracture surface indicated the presence of a conductive core (Figures 10 and 11). Although the core conductivity was not quantified in situ, the original suspension conductivity was $54 \mathrm{mS} / \mathrm{cm}$. Decreasing the diameter of the outer (sheath) needle from $16 \mathrm{G}$ to $17 \mathrm{G}$ (Figure 12) while retaining a $22 \mathrm{G}$ core needle, produced coaxial fibers with average diameters of $6.1 \mu \mathrm{m}$ and $3.3 \mu \mathrm{m}$ respectively, while the core diameter remained in the range of 1.1 to $1.9 \mu \mathrm{m}$. Image analysis was performed to quantify the alignment, density and diameter of the core-sheath fibers, and compared to pure PLLA fibers. The statistical alignment of the coaxial fibers, while good, was less that the single PLLA strands, mostly due to more cross strands that affected the standard deviation. The coaxial fiber process required higher flowrates, and correspondingly higher electric field strengths. The subsequent generation of stray electrical field is 
probably responsible for the less uniform spinning we saw. Mechanical testing to determine the Young's modulus and the hardness of the fibers was performed using a nanoindentation device. The results indicate that the PLLA sheathed fibers have a substantially lower modulus that the pure PLLA strands. This may be due to the higher flexibility of the thinner PLLA sheaths, or because the nano-indenter penetration depth was on the order of the outer sheath thickness $(\sim 1.5 \mu \mathrm{m})$

One of the motivations for this study was the production of insulated, electrically conducting nano/micro-wires for use in applications ranging from microelectronics to electrically stimulated tissue scaffolds. We have demonstrated for the first time, the electrospinning of highly-aligned fibers with an insulating sheath and a conductive core. The process discussed here utilizes PEDOT:PSS nanoparticles in a suspension of oleic acid. Improved core materials are needed, but alternative conductive polymers for the core of the coaxial fibers are limited due to their poor solubility in most common solvents. Spinning more highly aligned fiber mats while reducing cross fibers and stray currents would also be important for making tissue scaffolds for in vitro and in vivo cell cultures. This might be done by increasing the rotation rate or the diameter of the collection wheel or using other methods of producing aligned fiber scaffolds (i.e. parallel electrodes). Direct contact probes for measuring electrical conductivity in an FE-SEM or TEM are now available, and should be used to quantify the core conductivity in situ. The nano-engineering of conductive core/sheath fibers that can be used as microconnectors, neuro-electrical measurements tools, tissue scaffolds, and in microelectronics are obvious offshoots from this work. 


\section{In Vitro Chick Dorsal Root Ganglia Studies on PEDOT/PLLA Core/Sheath Microfiber Scaffolds}

\subsection{Abstract}

The formation of electrospun microfiber scaffolds with a core-sheath structure has been previously reported. These fibers had a conductive poly $(3,4-$ ethylenedioxythiophene):poly(styrene sulfonate) (PEDOT:PSS) core and a poly-L-lactic acid (PLLA) insulating sheath. To test the ability of the scaffolds to promote axonal outgrowth in vitro, cell studies were performed using stage E9 chick dorsal root ganglia (DRG) cells. Cells were cultured for 5 and 7 days on pure PLLA fibers, coaxial fibers with PEDOT:PSS core in the core, coaxial fibers with PEDOT:DBSA nanoparticles in the core, and coaxial fibers with only an oleic acid core. Cells were also cultured on two different diameter coaxial fibers spun with a 22G/16G spinneret combination and a 22G/17G spinneret combination (inner/outer). Some cells were cultured while being exposed to electrical stimulation of $23.9 \mathrm{mV}$ for the first 18 hours of culture. The initial studies indicated that in our successful cultures, the axonal extension was similar to that on single PLLA fibers; however, electrical stimulation during culture was not beneficial. Several cultures were not successful in promoting axonal outgrowth, indicating the core material and culture conditions for coaxial fibers needs further research.

\subsection{Introduction}

It has previously been shown that aligned PLLA fibers can aid in axonal outgrowth both in vitro and in vivo ${ }^{20,34}$. However, while fiber scaffolds have been shown 
to aid in extensive tissue regeneration in a rat in vivo model, the functional recovery of hind limb motor function has not been demonstrated ${ }^{20}$. It may be necessary to use a combined strategy approach to improve on the in vivo results that were seen in the study by Hurtado et al. In their study, axonal regeneration of a spinal cord injury in rats was complete at the proximal end but a gap persisted at the distal end. Combining the topographical guidance of aligned fiber scaffolds with biochemical or electrical stimuli may prove to be beneficial in completing distal axonal outgrowth, thereby improving motor function.

Previously, electrical stimulation has been shown to aid in axonal outgrowth both in vitro and in vivo ${ }^{76,77,78,79,83}$. In some cases, electrical stimulation with no topographical guidance cues has been shown to aid in axonal outgrowth, while some researchers, such as Koppes and Adams, have shown that when cells are cultured on random fiber mats and then exposed to electrical stimulation, axonal outgrowth can again be enhanced ${ }^{73,77}$. Koppes et al. demonstrated their results on aligned fiber scaffolds and after only 24 hours of culture and electrical stimulation showed initial axonal outgrowth was achieved ${ }^{77}$. Some of these studies were performed on conductive polymer structures, but the scaffolds were not aligned. It is our hypothesis that by combining aligned fiber scaffolds with conductive polymers we can in effect create an "artificial axon" and simulate an environment where electrical stimulation might enhance axonal outgrowth.

To further the understanding of axonal interaction with different microfiber scaffold materials and to improve functional recovery in vivo, we demonstrated the formation of coaxial fiber scaffolds with a conductive, PEDOT:PSS core and an 
insulating PLLA sheath. The mechanical and chemical properties of the fibers were studied, as well as the alignment, density, and diameter of the fiber scaffolds. Fibers were spun using a modified coaxial electrospinning technique. Using different sizes for a dual spinneret, fibers of different diameters were spun. Fibers spun using a 22G/16G spinneret set-up and a $22 \mathrm{G} / 17 \mathrm{G}$ spinneret set-up created fibers of 6.1 and $3.3 \mu \mathrm{m}$, respectively (inner/outer).

\subsection{Materials and Methods}

\subsubsection{Coaxial Fiber Formation}

Electrospinning fibers has been demonstrated previously (Chapter 4). The methodology is extensively outlined in Chapter 4 . All of the mechanical and chemical methods and results, as well as the results of all fiber characterization, are also outlined in Chapter 4.

\subsubsection{Dorsal Root Ganglia (DRG) Isolation and Culture}

Cell cultures were performed in accordance with the Michigan Technological University Institutional Animal Care and Use Committee (IACUC). Stages and coverslips prepared with fiber samples were sterilized using an ethylene oxide sterilization chamber. Samples were placed in a sterilization bag with a vial of ethylene oxide. The bag was purged of air, the vial broken, and the samples placed in the chamber for 12 hours. All DRG isolation and sterilization procedures are outlined in detail in Chapter 3. In short, chick DRG cells were isolated from embryos that were incubated for 9 days. The cells were seeded on various fiber scaffolds cultured for 5 and 7 days. 


\subsubsection{Immunochemistry Staining}

The staining procedures are detailed in Chapter 3. Briefly, after culture times were complete, a blocking buffer of $1 \%$ BSA in PBS was used to stop protein binding during staining. Primary TUG1 antibody was added to the cells and the cells were placed in the refrigerator overnight. The cells were washed and stained with secondary goat anti-mouse IgG antibody. The antibody was left to incubate on the cells at room temperature for 40 minutes. The cells were rinsed, and a DAPI stain was added to stain for glial cells. The DAPI stain was allowed to sit for 10 minutes. The cells were rinsed and attached to coverslips for imaging.

\subsubsection{Electrical Stimulation}

Electrical stimulation testing was done on some of the cell cultures to determine if the electrical cues in addition to the topographical cues would further aid in axonal outgrowth. The cover of a 6-well plate was modified with carbon electrodes. Slits were cut in the top of the cover where the cover fits over the wells in the culture plate. The carbon electrodes were slid in the slits and fit snugly. Six electrodes, for a total of three wells that could be stimulated, were fit into the cover. The electrodes were connected to a Keithly Instruments 225 current course microcurrent device (Figure 5-1). Testing was done to determine the appropriate level of current to supply. The first time the stimulation chamber was used the power supply was set to $3 \mathrm{~mA}$, which supplied $1 \mathrm{~mA}$ of current per well, and the cells stimulated for 24 hours. However, that current setting caused an overload of the supplied voltage ( $>1 \mathrm{~V})$ and the medium evaporated from the cells. The 
final setting was $12 \mu \mathrm{A}$ of current to the electrodes, which supplies $4 \mu \mathrm{A}$ per cell. After the cells were seeded on the samples and allowed to sit for four hours before adding medium, the power supply was turned on. The cells were stimulated for 18 hours. A multimeter connected to the power supply read $23.0 \mathrm{mV}$ of voltage supplied to the cells. After 18 hours, the power supply was turned off and the cells allowed to culture until five total days of culture were completed. Some of the electrical stimulation testing was performed on cells that were cultured on fiber scaffolds that were collected using the elevated stages described in Chapter 3, while other testing was done on cells cultured on fiber scaffolds collected on flat coverslips. 

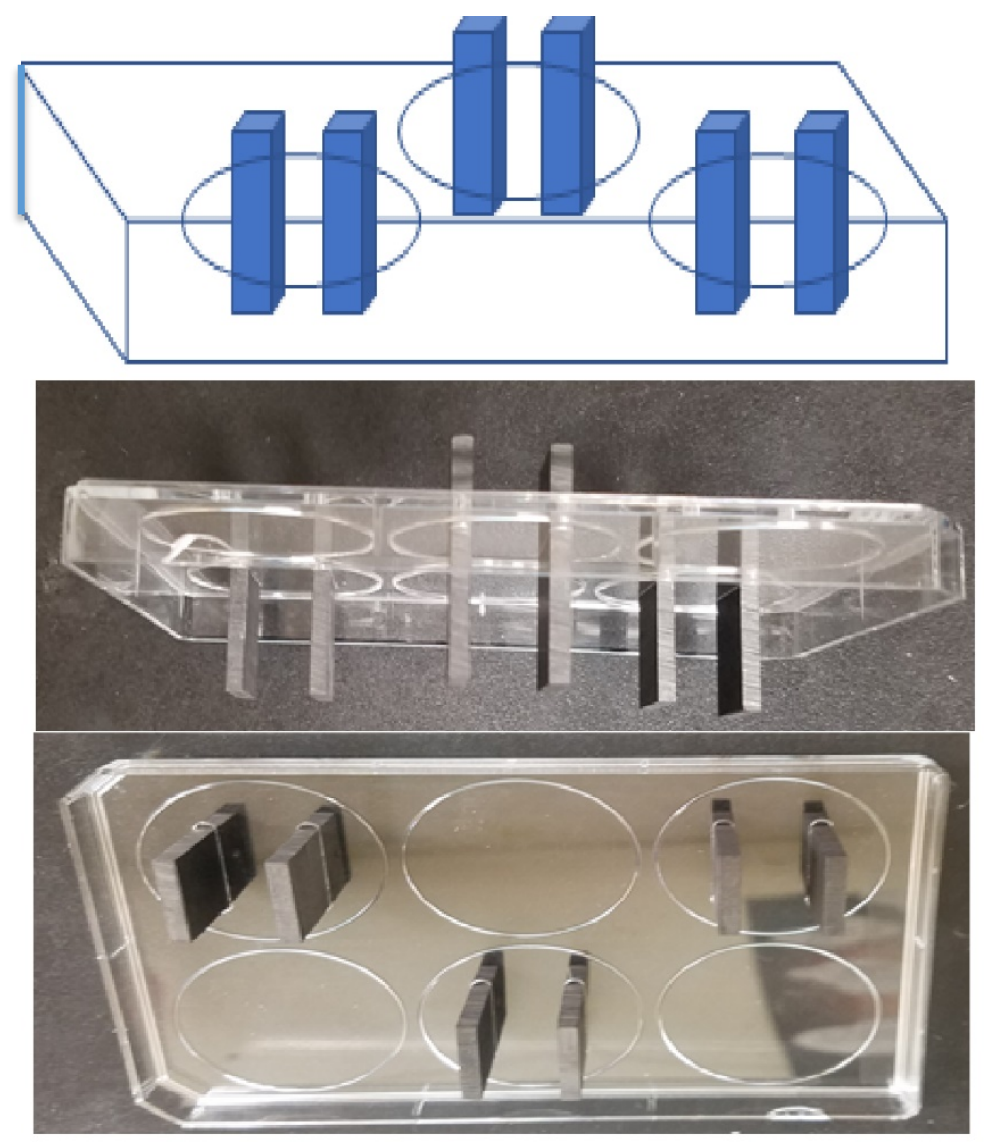

Figure 5-1:Drawing and working prototype of stimulation cover.

\subsection{Results and Discussion}

\subsubsection{In Vitro Tissue Cultures}

Five sets of in vitro cell cultures were performed using stage E9 chick dorsal root ganglia (DRG) cells. These cultures were performed to study the interactions of neural cells with the coaxial fibers, compare axonal outgrowth on the coaxial fibers with pure PLLA fibers, and determine biocompatibility of the coaxial fibers. All studies were performed using pure PLLA fibers as a control. Coaxial fibers with only oleic acid in the core were used as a control for core-sheath fibers to determine the effect of the 
PEDOT:PSS and PEDOT:DBSA in the core on toxicity and axonal outgrowth. Some of the DRG cell cultures were performed in the presence of electrical stimulation to determine if additional axonal outgrowth would be observed using the designed electrical stimulation cover (Figure 5-1).

Results of the studies can be seen in Figures 5-2, 5-3 and 5-4. Figure 5-2 shows the results of the first study performed on coaxial fibers. Figure 5-2A is a 5-day cell culture on pure PLLA. Figure 5-2B is a 7-day cell culture on coaxial fibers with PEDOT nanoparticles in the core. Figure 5-2C is a 7-day cell culture on coaxial fibers with PEDOT:PSS in the core. Figure 5-2D is the DAPI staining showing the proliferation of glial cells of Figure 5-2C. Figure 5-2E is a 5-day cell culture on coaxial fibers with PEDOT:PSS in the core. All the coaxial fibers in Figure 5-2 were spun with the $22 \mathrm{G}-16 \mathrm{G}$ needle combination, and were performed on scaffolds collected on flat coverslips. The green stain shows tubulin staining indicating axonal outgrowth. The blue staining is showing the DAPI stain for cell nuclei. Since axons do not have nuclei, in the case of Figures 5-2, 5-3, and 5-4, the blue DAPI stain is showing the proliferation of glial cells. This indicates that the axons are supported by the glial cells, showing successful axonal extension.

Figure 5-3A shows a 7-day culture on fibers with a PEDOT:PSS core and a large diameter fiber. Figure 5-3B shows a 5-day culture on fibers with a PEDOT:PSS core and a small diameter fiber (average $=3.3 \mu \mathrm{m}$ ). Figure $5-3 \mathrm{C}$ shows a 5 -day culture on fibers with a PEDOT:PSS core and a large diameter (average $=6.1 \mu \mathrm{m})$. Figure 5-3D shows a 5day culture on coaxial fibers with only an oleic acid core. All of the scaffolds in Figure 5- 
3 were spun on fiber scaffolds collected on a flat coverslip. While some of these cultures indicated glial cell proliferation by showing blue DAPI staining (Figures 5-3B and 5-3C), none of these cultures showed green tubulin staining that would indicate axonal extension.

Figure 5-4 shows the results of the electrical stimulation studies. Figure 5-4A shows a cell that received 24 hours of stimulation and was cultured for 24 hours on an elevated stage with a PEDOT:PSS core. Figure 5-4B shows the blue DAPI stain of 5-4A, indicated glial cell proliferation. Figures 5-4A and 5-4B show one of the cells cultured during the initial cell culture in which the voltage was set too high and evaporated the medium, so the cell was only cultured for 24 hours. Figure 5-4C shows a cell that received 18 hours of stimulation and was cultured for 5 days on pure PLLA fibers on a coverslip. Figure 5-4D shows a cell that received 18 hours of stimulation and was cultured for 5 days on coaxial fibers with a PEDOT:PSS core spun on a coverslip. 


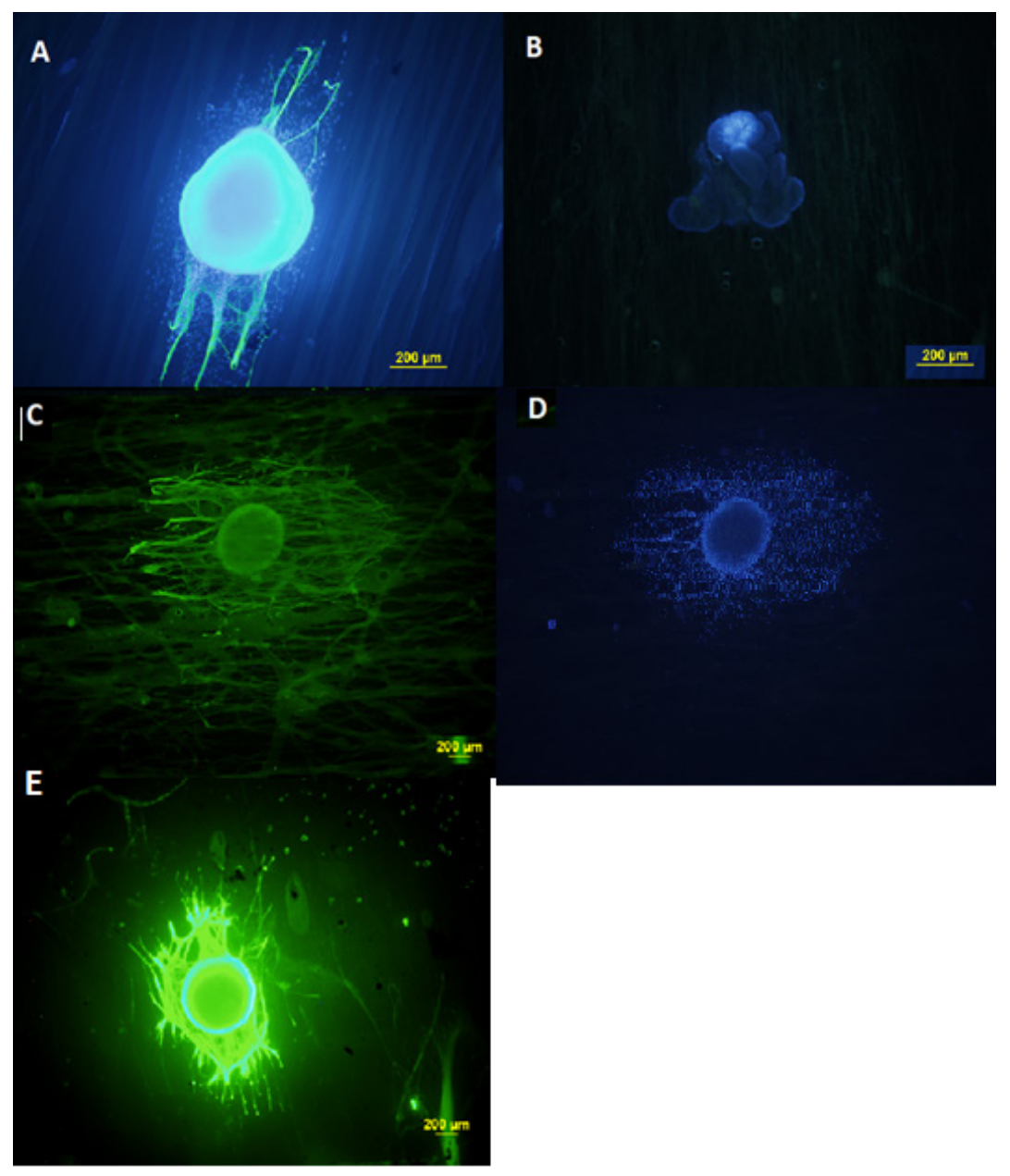

Figure 5-2: Results of first round of chick DRG cell cultures on coaxial fibers. (A) 5day DRG on pure PLLA (B) 7-day DRG on coaxial fibers with PEDOT nanoparticles (C) 7-day DRG on coaxial fibers with PEDOT:PSS (D) Glial stain of 5-2C (E) 5-day DRG on coaxial fibers with PEDOT:PSS 


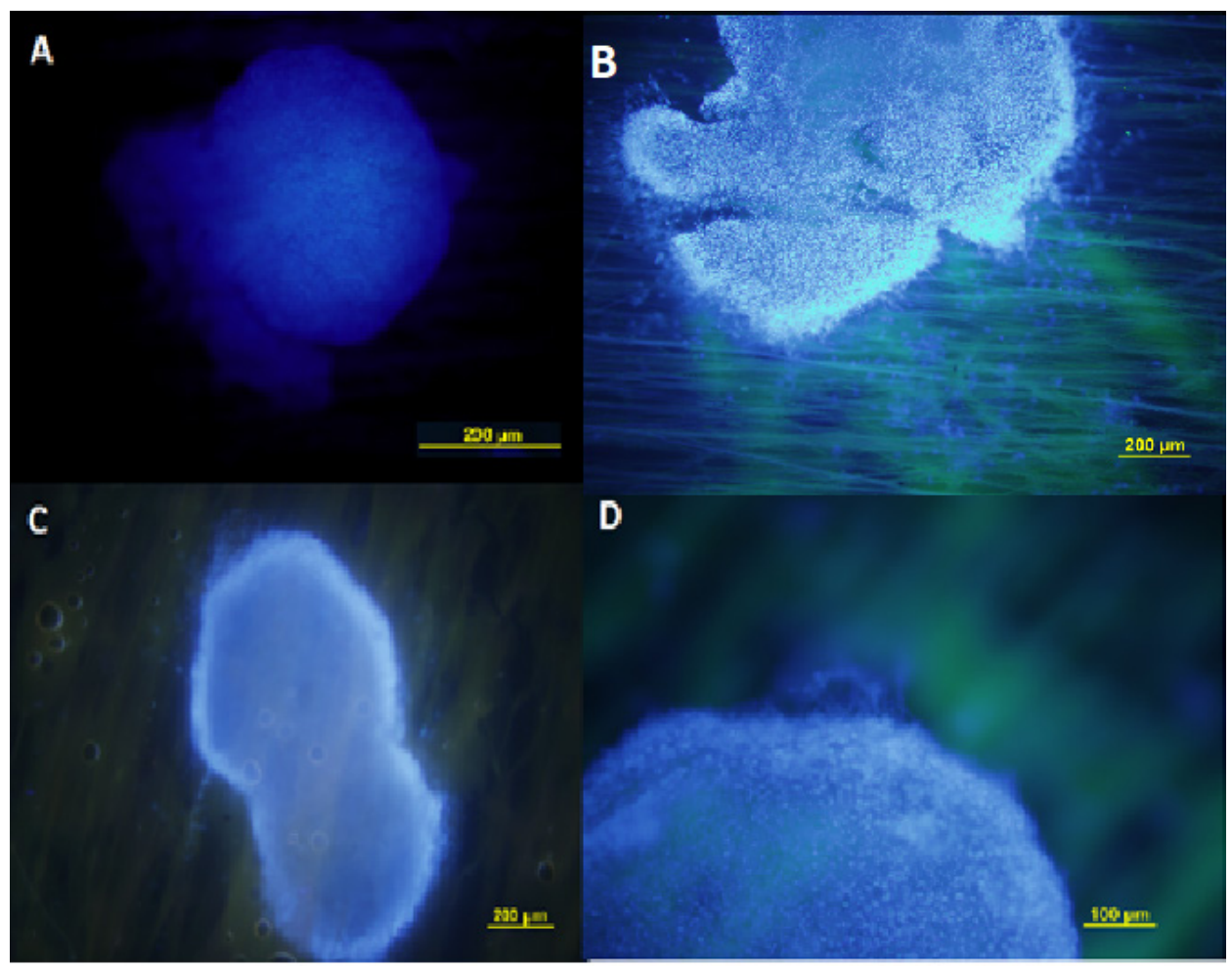

Figure 5-3: Results from subsequent cell studies. Axonal outgrowth was not enhanced. (A) 7-day culture on fibers with PEDOT:PSS core and large diameter (B) 5-day culture on fibers with a PEDOT:PSS core and small diameter (C) 5-day culture on fibers with a PEDOT:PSS core and large diameter (D) 5-day culture on fibers with oleic acid core 


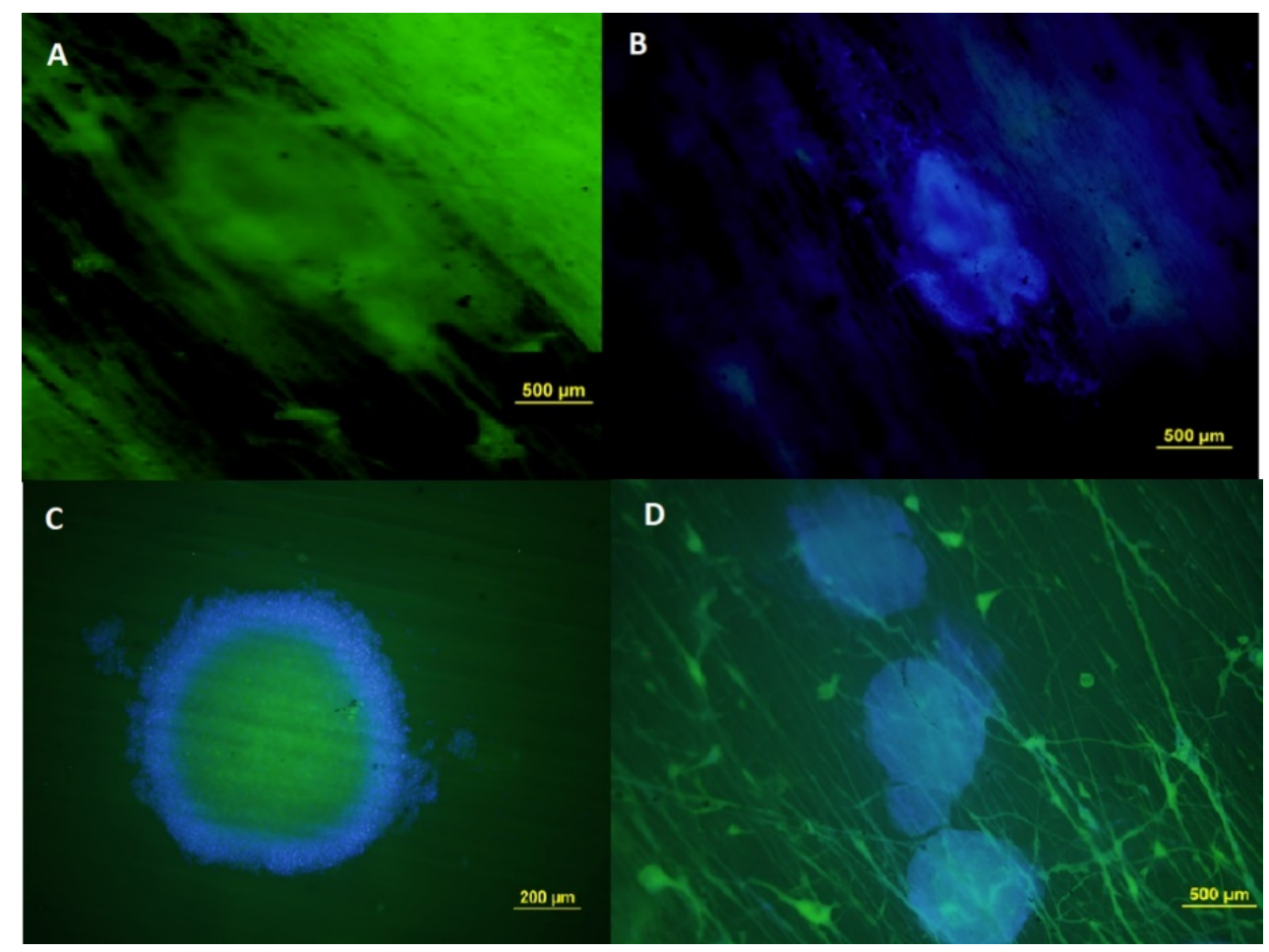

Figure 5-4: Results from electrical stimulation studies. (A) 24-hour stimulation and culture on stage, coaxial fibers with PEDOT:PSS core (B) Glial stain of A (C) 18-hour stimulation and 5-day culture on PLLA, on coverslip (D) 18-hour stimulation and 5day culture

Multiple DRG cultures were performed on the coaxial fibers using both the large diameter and the small diameter fibers, and compared to pure PLLA fibers. Initial cell studies compared the fibers containing the synthesized PEDOT:PSS in the core with the fibers that contained PEDOT:DBSA nanoparticles in the core. The DBSA counter ion in the PEDOT nanoparticles can be toxic to cells and did not aid in axonal outgrowth. The DRG cells cultured on fibers containing DBSA appeared to die (Figure 5-2B). The initial study with the PEDOT:PSS coaxial fibers appeared to aid in axonal outgrowth, but after 
performing multiple studies after, the results were never able to be replicated. Possible explanations include: (1) the fibers in the initial study were not perfectly coaxial, (2) fiber diameter was too large $(22 \mathrm{G} / 16 \mathrm{G}$ needle system, average diameter $=6.1 \mu \mathrm{m})$, and $(3)$ residual solvent on the fibers was inhibiting axonal outgrowth. A $22 \mathrm{G}-17 \mathrm{G}$ inner-outer system was used to smaller diameter fibers to test if the fiber diameter was inhibiting axonal outgrowth. A drying step was added to the electrospinning procedure to remove residual chloroform that may have been present on the fibers. After spinning, the samples were dried in a vacuum oven at $50^{\circ} \mathrm{C}$ for 30 minutes. However, these steps did not appear to assist the scaffolds in facilitating axonal outgrowth (Figure 5-3).

The initial positive cell results and studies by other investigators indicate that PEDOT:PSS does not have a toxic effect on cells ${ }^{95}$. It is possible the smaller diameter fibers are still too large to aid in axonal outgrowth $(3.3 \mu \mathrm{m})$, or the oleic acid core is causing a hindrance to the axonal outgrowth. It is also possible that the cross fibers causing the large deviation from alignment that was observed (Table 4-2) is hindering the axonal outgrowth. Wang et al. demonstrated that when axons encounter crossed fibers in the scaffold, outgrowth is stopped ${ }^{34}$.

More cell studies need to be performed, perhaps with a different culture methodology, different conductive polymer, or different core solvent. The lack of ability to replicate the initial positive results may indicate an issue with the culture methodology, as pure PLLA cultures did not show positive axonal extension as they had previously. Using a solvent other than oleic acid with PEDOT:PSS may create a more uniform dispersion of the PEDOT:PSS particles. If the PEDOT:PSS particles are more uniformly 
dispersed and are smaller, spinning needles with smaller diameters can be used to create smaller diameter fibers. Coaxial fibers with a smaller diameter can also be spun by perhaps spinning at a slightly higher voltage, or lower flow rates. More aligned scaffolds can also be spun by increasing the rotation rate of the spinning wheel. Adding electrical stimulation did not assist in outgrowth. One sample appeared to show some initial outgrowth but once again, this result was never replicated (Figure 5-4A). There seems to be something inherent in either the fibers or the cell culture procedure that is limiting the axonal outgrowth on the scaffolds.

\subsection{Conclusions and Recommendations}

Chick DRG cell cultures were performed on aligned, coaxial, core-sheath fiber structures with a conductive poly(3,4-ethylenedioxythiophene) (PEDOT) core and an insulating PLLA sheath. This structure created a type of "artificial axon" that could be used to supply electrical stimulation along the fibers during in vitro cell culture to provide multiple types of stimuli to promote axonal extension. Scaffolds were spun with either PEDOT:DBSA nanoparticles or synthesized PEDOT:PSS suspension in oleic acid as the core. Fibers with an average diameter of $6.1 \mu \mathrm{m}$ spun were with a $22 \mathrm{G} / 16 \mathrm{G}$ (inner/outer) spinning needle combination and fibers with an average diameter of $3.3 \mu \mathrm{m}$ were spun with a $22 \mathrm{G} / 17 \mathrm{G}$ spinning needle combination. Fibers of different diameters were used to determine the effect of fiber diameter on the ability of the scaffolds to promote axonal extension. Cells were also seeded on fiber scaffolds of pure PLLA and coaxial fibers with 
a plain oleic acid core to compare to cells that were seeded on the conductive, coaxial fibers.

Initial cell studies performed on the coaxial fibers with PEDOT:PSS cores indicated the coaxial fibers promoted axonal extension similar to that seen on pure PLLA fiber scaffolds. The cells seeded on fibers with PEDOT:DBSA nanoparticles in the core of the fibers appeared to die, as DBSA can be toxic to cells. PEDOT:DBSA particles were not used in the core of the coaxial fibers in the subsequent studies. The results of the initial study on PEDOT:PSS fibers were never replicated, despite performing the cultures multiple times. While it appeared the DAPI stain showed glial cell spreading that was at times extensive, tubulin staining did not show axonal outgrowth.

Fiber scaffolds with a PEDOT:PSS core were spun on both elevated stages and flat coverslips and used in electrical stimulation studies to determine if the additional stimulation would assist in axonal extension. Pure PLLA fibers were spun on flat coverslips and were also used in the electrical stimulation studies as controls. Cells were exposed to $23.9 \mathrm{mV}$ of stimulation for 18 hours. The stimulation was then removed and the cells kept in culture for a total of 5 days.

The electrical stimulation experiments did not suggest that the additional stimulation was beneficial to promoting axonal extension. The initial experiment showed some initial extension. However, the stimulation that was supplied during the first study was much too high, greater than $1 \mathrm{~V}$. This caused the culture medium to evaporate, and the cells were removed from culture after 24 hours. Further studies were performed at a 
much lower voltage, $23.9 \mathrm{mV}$, for 18 hours of stimulation and then 5 total days of culture did not show tubulin staining indicating axonal outgrowth. These studies also did not show DAPI staining that indicated glial cell proliferation.

\section{$\underline{\text { Recommendations }}$}

Further experiments need to be performed to replicate and improve upon the early results from this study. Greater success might be achieved by:

- Spinning fiber scaffolds with a higher degree of alignment. This can be done increasing the speed of rotation of the collection wheel, or looking at other methods to create aligned fiber scaffolds (i.e. parallel plates).

- Using a different core solvent than oleic acid for the PEDOT:PSS core suspension may prove beneficial. The PEDOT:PSS is not very easily dispersed in oleic acid and forms large clumps that may be affecting the size of the fibers. A solvent that the PEDOT:PSS is soluble in, or creates a more uniform suspension with smaller PEDOT:PSS particles, will create more uniform distribution in the core of the polymers and may help reduce the size.

- If smaller particles are present in the core, smaller diameter spinning needles can be used to create smaller diameter fibers. In the present study, the smallest diameter fibers that were spun had an average diameter of $3.3 \mu \mathrm{m}$. Previous studies indicated that the best in vitro culture results were performed on fibers that were around $2 \mu \mathrm{m}$ in diameter ${ }^{35}$. Creating microfiber scaffolds with a smaller diameter may better promote axonal extension. 
- Different methodologies for suppling electrical stimulation, such as different voltages, should be explored. Perhaps using electrical pulses rather than supplying a steady current for a given period would produce better results. A delayed stimulation after allowing the cells to culture for a day or longer may also improve the results.

Electrospun polymer fiber scaffolds have been shown to promote axonal extension and promote neural tissue regeneration. We hypothesize that by combining the topographical cues provided by the aligned scaffolds with electrical stimulation we can create a multifunctional fiber that can improve results previously seen and improve motor function in spinal cord injuries (SCI). Studies to better understand the interactions of cells with the fiber scaffolds and how cells respond to electrical stimulation need to be performed to improve the possibilities of clinical success of SCI repair. 


\section{Overall Conclusions and Recommendations for Future Work}

\subsection{Conclusions}

The work in this dissertation was performed to: (1) examine the literature to study the research of the use of electrospun fiber scaffolds to enhance axonal outgrowth, (2) examine the interactions of nerve cells with electrospun fiber scaffolds and answer previous criticisms about former research by our group, and (3) determine the parameters for electrospinning coaxial fiber scaffolds and examine the solution and fiber properties of coaxial fibers. The interactions of neural cells with coaxial fibers was also examined.

Chapter 2 lays out the review of the literature that was done to compile the work done by others in the field of electrospinning tissue scaffolds, and to examine gaps in the literature. It was determined that while much work has been done to perfect the parameters of electrospinning, study various polymers and biochemical agents and investigate the use of more complex fiber structures, research was missing that combined core-sheath fibers with a conductive polymer in the core and an insulating sheath to create a type of artificial axon. It was also determined that while some partially successful in vivo tests have been performed and reported, further investigation as to the interactions between neural tissue and implanted fiber scaffolds and ways to improve on the in vitro and in vivo success needs to be performed. 
The examination of the literature in Chapter 2, as well as criticisms of previous work that had been performed, led to the work that is explained in Chapter 3. Previously, Wang et al. had shown that aligned, electrospun PLLA fiber scaffolds could extensively assist in axonal outgrowth in an in vitro model ${ }^{34}$. Reviewers commented that a possibility for the in vitro success observed was due more to the restrictions caused by spinning the fibers on a flat, two-dimensional coverslip than the topographical cues provided by the fibers themselves. By spinning the fibers on a flat surface and then performing tissue cultures, the axons are restricted to the $2 \mathrm{D}$ plane and may be falling in the channels between the fibers, becoming trapped in the channels and growing outwards along them. If this is the case, the axons are not truly adhering to the fibers and following the alignment of the fiber surface. After testing different designs, a three-dimensional elevated stage was made using 3D-printing and machining techniques to both address these comments and provide an in vitro model to study in vivo interactions of axons with the fiber scaffolds. The stage had columns of $3 \mathrm{~mm}$ height and were $10 \mathrm{~mm}$ apart in distance. The elevated stage provided a 3D in vitro tissue culture environment and removed the constraint of the $2 \mathrm{D}$ surface that was used previously. Also, by creating a 3D tissue environment for in vitro studies, the in vivo environment is more closely replicated and provides insight to how the scaffolds interact with the tissues when implanted in vivo.

Examination of the fiber scaffolds spun on the elevated stages indicated that they did not differ greatly from PLLA scaffolds spun on flat coverslips in diameter or in alignment (Table 3-1). The results of the in vitro stage E9 chick dorsal root ganglia (DRG) studies in Chapter 3 indicated that removing the 2D environment did not have a 
negative effect on the ability of the fiber scaffolds to assist in axonal outgrowth (Table 32, Figure 3-7). Cell studies performed in vitro on both PLLA scaffolds spun on the elevated stages and on flat coverslips were compared. The elevated scaffolds still showed extensive axonal outgrowth. These results show that the axons attach to the PLLA fibers and the aligned scaffolds provide topographical guidance for axonal outgrowth. These results also indicate that scaffolds implanted in vivo would guide axonal outgrowth in the biological environment.

The work in Chapter 4 looked to address the mixed results of Hurtado et $\mathrm{al}^{20}$. Their work on an in vivo rat model looked at repairing the severed spinal column of rats with scaffolds made from aligned PLLA fibers. While extensive tissue regeneration in the spinal column was observed, the results of hind limb motor function testing did not indicate full functional recovery ${ }^{20}$. Although at the proximal end of the nerve injury regeneration was mostly complete, at the distal end, a gap remained after the axonal outgrowth had reached its maximum. Additional stimuli of the distal end may improve outgrowth across this gap. It was hypothesized that in the complex in vivo environment, multiple types of stimulation may be required to fully repair spinal cord injuries. The scaffolds implanted in the Hurtado study only supplied topographical cues. Additional stimuli may be necessary for repair of these injuries. Chapter 4 looked at electrospinning aligned coaxial, core-sheath microfiber scaffolds with a conductive core and an insulating sheath. Fibers of this structure provide the ability of the scaffolds to supply two sources of stimuli: electrical and topographical. By supplying an electrical current along the 
conductive fibers during in vitro cell cultures, electrical cues can be provided to the axons during outgrowth and may further assist in regeneration and repair.

Extensive analysis was performed to determine the best coaxial nozzle design to use, what proper flow rates for the two solutions were, the proper solvent to act as medium for the conductive core, the correct viscosity and concentration of the PLLA sheath, and rotation rate of the wheel necessary to spin aligned fibers. The inner and outer solutions were colored with different colored fluorescent dyes for imaging and characterization purposes. Conductive polymer PEDOT:PSS was synthesized to use in the conductive core of the polymer. Oleic acid provided the most consistent coaxial fibers. Different spinning needle combinations were used to create coaxial fibers of different diameters to determine the effect of the fiber diameter on axonal outgrowth during in vitro tissue culture. FE-SEM imaging was used to determine if the fibers were coaxial in nature. The FE-SEM imaging was also performed to determine if the fibers had a conductive core and insulating sheath. FTIR analysis was also performed to determine if the fibers were coaxial through chemical analysis of the surface. Mechanical testing was performed to determine the Young's modulus and hardness of the fibers to determine if they were suitable for tissue culture.

PEDOT:PSS polymer was successfully synthesized (Figure 4-5). Coaxial fibers of different diameters were spun (Table 4-2 and Figure 4-12). The fibers were coaxial in nature and had a conductive core with an insulating sheath (Figures 4-9, 4-10 and 4-11). Chapter 5 lays out the in vitro work that was performed to study the interactions of neural cells with both the coaxial fibers and electrical stimulation. An electrical stimulation 
cover was designed to provide stimulation to cells in a 6-well culture plate during in vitro tissue culture (Figure 5-1). Initial in vitro chick DRG cell studies indicated that the coaxial fiber scaffolds aided in axonal outgrowth and the PEDOT:PSS in the core was not toxic to the cells during culture (Figure 5-2). However, subsequent cultures on both the small and large diameter fibers did not show significant axonal outgrowth (Figure 5-3). Electrical stimulation studies also did not appear to assist in axonal outgrowth (5-4). It is possible the fiber diameters were too large, or the fiber scaffolds did not have a high enough degree of alignment to allow the fibers to provide enough guidance to the axons during culture.

The overall conclusions of this dissertation are as follows:

(1) Removing the 2D constraint of fibers spun on a flat coverslip by spinning elevated fiber scaffolds did not hinder the ability of the fiber scaffolds to aid and direct axonal outgrowth.

(2) The model provided by the in vitro cultures on elevated fiber scaffolds in Chapter 2 indicate that in the complex, three-dimensional biological environment, aligned fiber scaffolds still provide topographical cues to guide axonal outgrowth along fiber alignment.

(3) Coaxial fibers of different diameters with a conductive core and insulating sheath were spun using modified electrospinning nozzles and parameters.

(4) Initial In vitro DRG cell cultures performed on the coaxial fibers showed similar axonal outgrowth to the pure PLLA fibers. However, subsequent tests, both with and 
without electrical stimulation, did not appear to aid in axonal outgrowth during both 5 and 7-day cultures.

\subsection{Recommendations for Future Work}

While the work in Chapter 5 did not demonstrate the ability of conductive coaxial core-sheath fibers with a PEDOT:PSS core and PLLA sheath to improve upon the axonal outgrowth seen with pure PLLA fibers. However, we still hypothesized that fiber scaffolds can act as a bridge across the glial scar tissue present in spinal cord injuries (SCIs) and that through further examination, can be used to assist in the repair and restoration of function after injury. More highly aligned coaxial fiber scaffolds should be spun so the alignment is closer to that of pure PLLA fiber scaffolds as shown in Chapter 3 (Table 3-1). Increasing the rotation speed or diameter of the aluminum wheel may allow for a higher degree of alignment. It is also possible to spin coaxial fibers with an even smaller diameter than what was shown in Chapter 4 (Table 4-2). Increasing the voltage or decreasing the inner and outer flow rates without changing the 1:3 inner to outer flow rate ratio that was shown to provide the best coaxial fibers can allow for smaller diameter fibers. It would be difficult to use a smaller diameter needle for the core solution than the $22 \mathrm{G}$ needle because clogging becomes an issue. Also, a needle smaller than $17 \mathrm{G}$ would be too small for the $22 \mathrm{G}$ needle to fit inside.

While the electrical stimulation did not appear to aid in axonal outgrowth, it is still our hypothesis that electrical stimulation can be used not only to provide additional stimuli to aid in axonal outgrowth, but can be used to manipulate the outgrowth and reconnections of the axons in vivo. It may be necessary to look at different conductive 
polymers. The suspension of polymer in the core can be improved to change the fibers in a way that improves the axonal outgrowth observed on the fiber scaffolds. While oleic acid in this study spun the most consistent coaxial fibers, a larger variety of solvents can be investigated. The coaxial nature of core-sheath fibers allows for a great deal of flexibility of core solvents to be used. The coaxial fiber structure can also be used with APIs such as nerve growth factor (NGF), either in addition to the conductive polymer or on their own, to improve axonal outgrowth. This dissertation did not look examine the effect of biochemical cues on axonal outgrowth. Placing NGF in the core of the fiber, either with a conductive polymer or without, or by placing NGF in the sheath with the PLLA and keeping the conductive polymer in the core, one can increase the number of stimuli that is being provided during axonal outgrowth and perhaps improve the outgrowth seen in the results of Chapter 5. Other biochemical agents or biopolymers, such as collagen or laminin, can be used to improve the biocompatibility of the fibers or to improve the axonal outgrowth.

Injuries to both the central nervous system (CNS) and peripheral nervous system (PNS) continue to be areas of extensive research. The lack of regenerative capabilities of neural tissue indicates the need for clinical interventions for repair of these injuries and restoration of neural function. The research in our work, like that of the literature, presented mixed results. Some electrospun fiber scaffolds aided in axonal outgrowth in vitro (Chapter 3), while other scaffolds did not appear to aid in axonal outgrowth of chick DRG cells in vitro (Chapter 5). Our results and those of other researchers indicate that 
more extensive research is needed to promote the clinical use of using electrospun fiber scaffolds to repair neural injuries. 


\section{References}

1. Tortora J, Derrickson B. Principles of Anatomy \& Physiology. Danvers, MA: Wiley; 2014. 1127 p.

2. Spinal Cord Injury (SCI) Facts and Figures at a Glance [Internet]. 2016 [updated cited

3. Wang HB. Development of an Implantable Multilayer 3D Scaffold Consisting of Aligned Electrospun Fibers for Neuronal Regeneration Applications. Houghton, Michigan: Michigan Technological University; 2008. 146 p.

4. Tom VJ, Steinmetz MP, Miller JH, Doller CM, Silver J. Studies on the development and behavior of the dystrophic growth cone, the hallmark of regeneration failure, in an in vitro model of the glial scar and after spinal cord injury. J Neurosci 2004;24(29):6531-9.

5. Navarro X, Vivo M, Valero-Cabre A. Neural plasticity after peripheral nerve injury and regeneration. Prog Neurobiol 2007;82(4):163-201.

6. Campbell WW. Evaluation and management of peripheral nerve injury. Clin Neurophysiol 2008;119(9):1951-65.

7. Kijenska E, Prabhakaran MP, Swieszkowski W, Kurzydlowski KJ, Ramakrishna S. Electrospun bio-composite P(LLA-CL)/collagen I/collagen III scaffolds for 
nerve tissue engineering. J Biomed Mater Res B Appl Biomater 2012;100(4):1093-102.

8. Rieichert P, Kilbowicz Z, Dziegiel P, Pula b, Wrzosek M, Bochenska A, Gosk J. Effect of collateral sprouting on donor nerve function after nerve coaptation a study of the brachial plexus. Medical Science Monitor 2016;22:387-396.

9. Schaub NJ, Johnson CD, Cooper B, Gilbert RJ. Electrospun Fibers for Spinal Cord Injury Research and Regeneration. J Neurotrauma 2016;33(15):1405-15.

10. Cheng H, Cao Y, Olson L. Spinal cord repair in adult paraplegic rats: partial restoration of hind limb function.pdf. Science 1996;273(5274):510-513.

11. Houle JD, Tom VJ, Mayes D, Wagoner G, Phillips N, Silver J. Combining an autologous peripheral nervous system "bridge" and matrix modification by chondroitinase allows robust, functional regeneration beyond a hemisection lesion of the adult rat spinal cord. J Neurosci 2006;26(28):7405-15.

12. Zustiak SP, Pubill S, Ribeiro A, Leach JB. Hydrolytically degradable poly(ethylene glycol) hydrogel scaffolds as a cell delivery vehicle: characterization of PC12 cell response. Biotechnol Prog 2013;29(5):1255-64.

13. Gungor-Ozkerim PS, Balkan T, Kose GT, Sarac AS, Kok FN. Incorporation of growth factor loaded microspheres into polymeric electrospun nanofibers for tissue engineering applications. J Biomed Mater Res A 2014;102(6):1897-908. 
14. Gu X, Ding F, Williams DF. Neural tissue engineering options for peripheral nerve regeneration. Biomaterials 2014;35(24):6143-56.

15. Zhu W, Masood F, O'Brien J, Zhang LG. Highly aligned nanocomposite scaffolds by electrospinning and electrospraying for neural tissue regeneration. Nanomedicine 2015;11(3):693-704.

16. Mobasseri A, Faroni A, Minogue BM, Downes S, Terenghi G, Reid AJ. Polymer scaffolds with preferential parallel grooves enhance nerve regeneration. Tissue Eng Part A 2015;21(5-6):1152-62.

17. Archibald SJ, Krarup C, Shefner J, Li ST, Madison RD. A collagen-based nerve guide conduit for peripheral nerve repair: an electrophysiological study of nerve regeneration in rodents and nonhuman primates. J Comp Neurol 1991;306(4):68596.

18. Mahoney MJ, Anseth KS. Three-dimensional growth and function of neural tissue in degradable polyethylene glycol hydrogels. Biomaterials 2006;27(10):2265-74.

19. Yao S, Liu X, Yu S, Wang X, Zhang S, Wu Q, Sun X, Mao H. Co-effects of matrix low elasticity and aligned topography on stem cell neurogenic differentiation and rapid neurite outgrowth. Nanoscale 2016;8(19):10252-65.

20. Hurtado A, Cregg JM, Wang HB, Wendell DF, Oudega M, Gilbert RJ, McDonald JW. Robust CNS regeneration after complete spinal cord transection using aligned poly-L-lactic acid microfibers. Biomaterials 2011;32(26):6068-79. 
21. He J-H, Liu Y, Mo L-F, Wan Y-Q, Xu L. Electrospun Nanofibres and Their Applications. United Kingdom: iSmithers; 2008.

22. Ingavle GC, Leach JK. Advancements in electrospinning of polymeric nanofibrous scaffolds for tissue engineering. Tissue Eng Part B Rev 2014;20(4):277-93.

23. Zeng J, Chen X, Xu X, Liang Q, Bian X, Yang L, Jing X. Ultrafine fibers electrospun from biodegradable polymers. Journal of Applied Polymer Science 2003;89(4):1085-1092.

24. Gupta P, Elkins C, Long TE, Wilkes GL. Electrospinning of linear homopolymers of poly(methyl methacrylate): exploring relationships between fiber formation, viscosity, molecular weight and concentration in a good solvent. Polymer 2005;46(13):4799-4810.

25. Andrady AL. Science and Technology of Polymer Nanofibers. New Jersey: John Wiley \& Sons; 2008.

26. Kakinoki S, Nakayama M, Moritan T, Yamaoka T. Three-layer microfibrous peripheral nerve guide conduit composed of elastin-laminin mimetic artificial protein and poly(L-lactic acid). Front Chem 2014;2:52.

27. He L, Tang S, Prabhakaran MP, Liao S, Tian L, Zhang Y, Xue W, Ramakrishna S. Surface modification of PLLA nano-scaffolds with laminin multilayer by LbL assembly for enhancing neurite outgrowth. Macromol Biosci 2013;13(11):1601-9. 
28. Jiang X, Mi R, Hoke A, Chew SY. Nanofibrous nerve conduit-enhanced peripheral nerve regeneration. J Tissue Eng Regen Med 2014;8(5):377-85.

29. Zamani F, Amani-Tehran M, Latifi M, Shokrgozar MA. The influence of surface nanoroughness of electrospun PLGA nanofibrous scaffold on nerve cell adhesion and proliferation. J Mater Sci Mater Med 2013;24(6):1551-60.

30. Lee JY, Bashur CA, Goldstein AS, Schmidt CE. Polypyrrole-coated electrospun PLGA nanofibers for neural tissue applications. Biomaterials 2009;30(26):432535.

31. Mohtaram NK, Ko J, Agbay A, Rattray D, Neill PO, Rajwani A, Vasandani R, Thu HL, Jun MBG, Willerth SM. Development of a glial cell-derived neurotrophic factor-releasing artificial dura for neural tissue engineering applications. J. Mater. Chem. B 2015;3(40):7974-7985.

32. Hardick O, Stevens B, Bracewell DG. Nanofibre fabrication in a temperature and humidity controlled environment for improved fibre consistency. Journal of Materials Science 2011;46(11):3890-3898.

33. Corey JM, Lin DY, Mycek KB, Chen Q, Samuel S, Feldman EL, Martin DC. Aligned electrospun nanofibers specify the direction of dorsal root ganglia neurite growth. J Biomed Mater Res A 2007;83(3):636-45. 
34. Wang HB, Mullins ME, Cregg JM, Hurtado A, Oudega M, Trombley MT, Gilbert RJ. Creation of highly aligned electrospun poly-L-lactic acid fibers for nerve regeneration applications. J Neural Eng 2009;6(1):016001.

35. Wang HB, Mullins ME, Cregg JM, McCarthy CW, Gilbert RJ. Varying the diameter of aligned electrospun fibers alters neurite outgrowth and Schwann cell migration. Acta Biomater 2010;6(8):2970-8.

36. Schaub NJ, Britton T, Rajachar R, Gilbert RJ. Engineered nanotopography on electrospun PLLA microfibers modifies RAW 264.7 cell response. ACS Appl Mater Interfaces 2013;5(20):10173-84.

37. Zuidema JM, Provenza C, Caliendo T, Dutz S, Gilbert RJ. Magnetic NGFreleasing PLLA/iron oxide nanoparticles direct extending neurites and preferentially guide neurites along aligned electrospun microfibers. ACS Chem Neurosci 2015;6(11):1781-8.

38. Dinis TM, Elia R, Vidal G, Auffret A, Kaplan DL, Egles C. Method to form a fiber/growth factor dual-gradient along electrospun silk for nerve regeneration. ACS Appl Mater Interfaces 2014;6(19):16817-26.

39. Xie J, Liu W, MacEwan MR, Bridgman PC, Younan X. Neurite Outgrowth on Electrospun Nanofibers with Uniaxial Alignment. ACS Nano 2014.

40. Huang C, Ouyang Y, Niu H, He N, Ke Q, Jin X, Li D, Fang J, Liu W, Fan C and others. Nerve guidance conduits from aligned nanofibers: improvement of nerve 
regeneration through longitudinal nanogrooves on a fiber surface. ACS Appl Mater Interfaces 2015;7(13):7189-96.

41. Bonani W, Motta A, Migliaresi C, Tan W. Biomolecule gradient in micropatterned nanofibrous scaffold for spatiotemporal release. Langmuir 2012;28(38):13675-87.

42. Subramanian A, Krishnan UM, Sethuraman S. Fabrication, characterization and in vitro evaluation of aligned PLGA-PCL nanofibers for neural regeneration. Ann Biomed Eng 2012;40(10):2098-110.

43. Prabhakaran MP, Vatankhah E, Ramakrishna S. Electrospun aligned PHBV/collagen nanofibers as substrates for nerve tissue engineering. Biotechnol Bioeng 2013;110(10):2775-84.

44. Kuihua Z, Chunyang W, Cunyi F, Xiumei M. Aligned SF/P(LLA-CL)-blended nanofibers encapsulating nerve growth factor for peripheral nerve regeneration. $\mathrm{J}$ Biomed Mater Res A 2014;102(8):2680-91.

45. Chew SY, Wen J, Yim EK, Leong KW. Sustained release of proteins from electrospun biodegradable fibers. Biomacromolecules 2005;6(4):2017-24.

46. Rim NG, Shin CS, Shin H. Current approaches to electrospun nanofibers for tissue engineering. Biomedical Materials 2013;8(1):014102. 
47. Bhattacharjee P, Kundu B, Naskar D, Maiti TK, Bhattacharya D, Kundu SC. Nanofibrous nonmulberry silk/PVA scaffold for osteoinduction and osseointegration. Biopolymers 2015;103(5):271-84.

48. Khajavi R, Abbasipour M, Bahador A. Electrospun biodegradable nanofibers scaffolds for bone tissue engineering. Journal of Applied Polymer Science 2016;133(3):n/a-n/a.

49. Xu T, Miszuk JM, Zhao Y, Sun H, Fong H. Electrospun polycaprolactone 3D nanofibrous scaffold with interconnected and hierarchically structured pores for bone tissue engineering. Adv Healthc Mater 2015;4(15):2238-46.

50. $\mathrm{Xu} \mathrm{H}, \mathrm{Li} \mathrm{H}, \mathrm{Ke} \mathrm{Q}$, Chang J. An anisotropically and heterogeneously aligned patterned electrospun scaffold with tailored mechanical property and improved bioactivity for vascular tissue engineering. ACS Appl Mater Interfaces 2015;7(16):8706-18.

51. Zhao G, Zhang X, Lu TJ, Xu F. Recent Advances in Electrospun Nanofibrous Scaffolds for Cardiac Tissue Engineering. Advanced Functional Materials 2015;25(36):5726-5738.

52. Zhang K, Zheng H, Liang S, Gao C. Aligned PLLA nanofibrous scaffolds coated with graphene oxide for promoting neural cell growth. Acta Biomater 2016;37:131-42. 
53. Mu Y, Wu F, Lu Y, Wei L, Yuan W. Progress of electrospun fibers as nerve conduits for neural tissue repair. Nanomedicine (Lond) 2014;9(12):1869-83.

54. Entekhabi E, Haghbin Nazarpak M, Moztarzadeh F, Sadeghi A. Design and manufacture of neural tissue engineering scaffolds using hyaluronic acid and polycaprolactone nanofibers with controlled porosity. Mater Sci Eng C Mater Biol Appl 2016;69:380-7.

55. Zhang Y, Huang J, Huang L, Liu Q, Shao H, Hu X, Song L. Silk Fibroin-Based Scaffolds with Controlled Delivery Order of VEGF and BDNF for Cavernous Nerve Regeneration. ACS Biomaterials Science \& Engineering 2016;2(11):20182025.

56. Jing X, Mi HY, Peng J, Peng XF, Turng LS. Electrospun aligned poly(propylene carbonate) microfibers with chitosan nanofibers as tissue engineering scaffolds. Carbohydr Polym 2015;117:941-9.

57. Zhao J, Liu H, Xu L. Preparation and formation mechanism of highly aligned electrospun nanofibers using a modified parallel electrode method. Materials \& Design 2016;90:1-6.

58. Dippold D, Cai A, Hardt M, Boccaccini AR, Horch R, Beier JP, Schubert DW. Novel approach towards aligned PCL-Collagen nanofibrous constructs from a benign solvent system. Mater Sci Eng C Mater Biol Appl 2017;72:278-283. 
59. Bhutto MA, Zhang J, Sun B, El-Hamshary H, Al-Deyab SS, Mo X. Development of poly (L-lactide-co-caprolactone) multichannel nerve conduit with aligned electrospun nanofibers for Schwann cell proliferation. International Journal of Polymeric Materials and Polymeric Biomaterials 2016;65(7):323-329.

60. Xu C. Aligned biodegradable nanofibrous structure: a potential scaffold for blood vessel engineering. Biomaterials 2004;25(5):877-886.

61. $\mathrm{Xu} \mathrm{Y,} \mathrm{Wu} \mathrm{Z,} \mathrm{Dong} \mathrm{X,} \mathrm{Li} \mathrm{H.} \mathrm{Combined} \mathrm{biomaterial} \mathrm{signals} \mathrm{stimulate}$ communications between bone marrow stromal cell and endothelial cell. RSC Adv. 2017;7(9):5306-5314.

62. Jenkins PM, Laughter MR, Lee DJ, Lee YM, Freed CR, Park D. A nerve guidance conduit with topographical and biochemical cues: potential application using human neural stem cells. Nanoscale Res Lett 2015;10(1):972.

63. Chen W, Li D, Ei-Shanshory A, El-Newehy M, Ei-Hamshary HA, Al-Deyab SS, He C, Mo X. Dexamethasone loaded core-shell SF/PEO nanofibers via green electrospinning reduced endothelial cells inflammatory damage. Colloids Surf B Biointerfaces 2015;126:561-8.

64. Merkle VM, Tran PL, Hutchinson M, Ammann KR, DeCook K, Wu X, Slepian MJ. Core-shell PVA/gelatin electrospun nanofibers promote human umbilical vein endothelial cell and smooth muscle cell proliferation and migration. Acta Biomater 2015;27:77-87. 
65. Jalaja K, Naskar D, Kundu SC, James NR. Potential of electrospun core-shell structured gelatin-chitosan nanofibers for biomedical applications. Carbohydr Polym 2016;136:1098-107.

66. Lu Y, Huang J, Yu G, Cardenas R, Wei S, Wujcik EK, Guo Z. Coaxial electrospun fibers: applications in drug delivery and tissue engineering. Wiley Interdiscip Rev Nanomed Nanobiotechnol 2016;8(5):654-77.

67. Su Y, Li X, Wang H, He C, Mo X. Fabrication and characterization of biodegradable nanofibrous mats by mix and coaxial electrospinning. J Mater Sci Mater Med 2009;20(11):2285-94.

68. Nguyen TT, Ghosh C, Hwang SG, Chanunpanich N, Park JS. Porous core/sheath composite nanofibers fabricated by coaxial electrospinning as a potential mat for drug release system. Int J Pharm 2012;439(1-2):296-306.

69. Wang CY, Liu JJ, Fan CY, Mo XM, Ruan HJ, Li FF. The effect of aligned coreshell nanofibres delivering NGF on the promotion of sciatic nerve regeneration. J Biomater Sci Polym Ed 2012;23(1-4):167-84.

70. Gluck JM, Rahgozar P, Ingle NP, Rofail F, Petrosian A, Cline MG, Jordan MC, Roos KP, Maclellan WR, Shemin RJ and others. Hybrid coaxial electrospun nanofibrous scaffolds with limited immunological response created for tissue engineering. J Biomed Mater Res B Appl Biomater 2011;99(1):180-90.

71. $<$ Comparative Medicine.pdf $>$. 
72. Al-Majed AA, Neumann CM, Brushart TM, Gordon T. Brief electrical stimulation promotes the speed and accuracy of motor axonal regeneration. The Journal of Neuroscience 2000;20(7):2602-2608.

73. Adams RD, Rendell SR, Counts LR, Papke JB, Willits RK, Harkins AB. Electrical and neurotrophin enhancement of neurite outgrowth within a 3D collagen scaffold. Ann Biomed Eng 2014;42(6):1282-91.

74. Kotwal A, Schmidt CE. Electrical stimulation alters protein absorption and nerve cell interactions with electrically conduction biomaterials. Biomaterials 2001;22:1055-1064.

75. Chang YJ, Hsu CM, Lin CH, Lu MS, Chen L. Electrical stimulation promotes nerve growth factor-induced neurite outgrowth and signaling. Biochim Biophys Acta 2013;1830(8):4130-6.

76. Wood MD, Willits RK. Applied electric field enhances DRG neurite growth: influence of stimulation media, surface coating and growth supplements. J Neural Eng 2009;6(4):046003.

77. Koppes AN, Zaccor NW, Rivet CJ, Williams LA, Piselli JM, Gilbert RJ, Thompson DM. Neurite outgrowth on electrospun PLLA fibers is enhanced by exogenous electrical stimulation. J Neural Eng 2014;11(4):046002. 
78. Xu C, Kou Y, Zhang P, Han N, Yin X, Deng J, Chen B, Jiang B. Electrical stimulation promotes regeneration of defective peripheral nerves after delayed repair intervals lasting under one month. PLoS One 2014;9(9):e105045.

79. Elzinga K, Tyreman N, Ladak A, Savaryn B, Olson J, Gordon T. Brief electrical stimulation improves nerve regeneration after delayed repair in Sprague Dawley rats. Exp Neurol 2015;269:142-53.

80. Tandon N, Cimetta E, Taubman A, Kupferstein N, Madaan U, Mighty J, Redenti S, Vunjak-Novakovic G. Biomimetic electrical stimulation platform for neural differentiation of retinal progenitor cells. Conf Proc IEEE Eng Med Biol Soc 2013;2013:5666-9.

81. Lee YS, Collins G, Arinzeh TL. Neural differentiation of human neural stem/progenitor cells on piezoelectric scaffolds. 2010:1-2.

82. Kim DH, Richardson-Burns SM, Hendricks JL, Sequera C, Martin DC. Effect of Immobilized Nerve Growth Factor on Conductive Polymers: Electrical Properties and Cellular Response. Advanced Functional Materials 2007;17(1):79-86.

83. Schmidt CE, Shastri VR, Vacanti JP, Langer R. Stimulation of neurite outgrowth using an electrically conducting polymer. Proc. Natl. Acad. Sci. 1997;94:89488953.

84. Shi Z, Gao H, Feng J, Ding B, Cao X, Kuga S, Wang Y, Zhang L, Cai J. In situ synthesis of robust conductive cellulose/polypyrrole composite aerogels and their 
potential application in nerve regeneration. Angew Chem Int Ed Engl 2014;53(21):5380-4.

85. Thunberg J, Kalogeropoulos T, Kuzmenko V, Hagg D, Johannesson S, Westman G, Gatenholm P. In situ synthesis of conductive polypyrrole on electrospun cellulost nanofibers: scaffold for neural tissue engineering. Cellulose 2015;22(3):1459-1467.

86. Zeng J, Huang Z, Yin G, Qin J, Chen X, Gu J. Fabrication of conductive NGFconjugated polypyrrole-poly(1-lactic acid) fibers and their effect on neurite outgrowth. Colloids Surf B Biointerfaces 2013;110:450-7.

87. Xu H, Holzwarth JM, Yan Y, Xu P, Zheng H, Yin Y, Li S, Ma PX. Conductive PPY/PDLLA conduit for peripheral nerve regeneration. Biomaterials 2014;35(1):225-35.

88. Cong Y, Liu S, Chen H. Fabrication of Conductive Polypyrrole Nanofibers by Electrospinning. Journal of Nanomaterials 2013;2013:1-6.

89. Yamato H, Ohwa M, Wernet W. Stability of Polypyrrole and Poly(3,4Ethylenedioxythiophene) for Biosensor Application. Journal of Electroanalytical Chemistry 1995;397(1-2):163-170.

90. Xiao Y, Martin DC, Xinyan C, Shenai M. Surface Modification of Neural Probes with Conducting Polymer Poly(hyrdocymethylated-2,4-ethylenedioxythipene) 
and its Biocompatibility. Applied Biochemistry and Biotechnology 2006;128:117129.

91. Zubair NA, Rahman NA, Lim HN, Zawawi RM, Sulaiman Y. Electrochemical properties of PVA-GO/PEDOT nanofibers prepared using electrospinning and electropolymerization techniques. RSC Adv. 2016;6(21):17720-17727.

92. Sebaa M, Nguyen TY, Dhillon S, Garcia S, Liu H. The effects of poly(3,4ethylenedioxythiophene) coating on magnesium degradation and cytocompatibility with human embryonic stem cells for potential neural applications. J Biomed Mater Res A 2015;103(1):25-37.

93. Luo S-C, Ali EM, Tansil NC, Yu H-h, Gao S, Kantchev EAB, Ying JY. Poly(3,4ethylenedioxythiophene) (PEDOT) Nanobiointerfaces: Thin, Ultrasmooth, and Functionalized PEDOT Films with in Vitro and in Vivo Biocompatibility. Langmuir 2008;24:8071-8077.

94. Yang J, Kim DH, Hendricks JL, Leach M, Northey R, Martin DC. Ordered surfactant-templated poly(3,4-ethylenedioxythiophene) (PEDOT) conducting polymer on microfabricated neural probes. Acta Biomater 2005;1(1):125-36.

95. Asplund M, Thaning E, Lundberg J, Sandberg-Nordqvist AC, Kostyszyn B, Inganas $\mathrm{O}$, von Holst $\mathrm{H}$. Toxicity evaluation of PEDOT/biomolecular composites intended for neural communication electrodes. Biomed Mater 2009;4(4):045009. 
96. Xie J, Macewan MR, Willerth SM, Li X, Moran DW, Sakiyama-Elbert SE, Xia Y. Conductive Core-Sheath Nanofibers and Their Potential Application in Neural Tissue Engineering. Adv Funct Mater 2009;19(14):2312-2318.

97. Zhang J, Qiu K, Sun B, Fang J, Zhang K, Ei-Hamshary H, Al-Deyab SS, Mo X. The aligned core-sheath nanofibers with electrical conductivity for neural tissue engineering. J. Mater. Chem. B 2014;2(45):7945-7954.

98. Xu Q, Li Y, Feng W, Yuan X. Fabrication and electrochemical properties of polyvinyl alcohol/poly(3,4-ethylenedioxythiophene) ultrafine fibers via electrospinning of EDOT monomers with subsequent in situ polymerization. Synthetic Metals 2010;160(1-2):88-93.

99. Angeles M, Cheng HL, Velankar SS. Emulsion electrospinning: composite fibers from drop breakup during electrospinning. Polymers for Advanced Technologies 2008;19(7):728-733.

100. Hurtado A, Gilbert RJ, Wang HB, Cregg JM, Mullins ME, Oudega M. Threedimensional scaffolds, methods for fabricating the same, and methods of treating a peripheral nerve or spinal cord injury. Google Patents; 2013.

101. Srouji S, Ben-David D, Lotan R, Livne E, Avrahami R, Zussman E. Slow-release human recombinant bone morphogenetic protein-2 embedded within electrospun scaffolds for regeneration of bone defect: in vitro and in vivo evaluation. Tissue Eng Part A 2011;17(3-4):269-77. 
102. Srinath D, Lin S, Knight DK, Rizkalla AS, Mequanint K. Fibrous biodegradable 1-alanine-based scaffolds for vascular tissue engineering. J Tissue Eng Regen Med 2014;8(7):578-88.

103. Castro NJ, Hacking SA, Zhang LG. Recent progress in interfacial tissue engineering approaches for osteochondral defects. Ann Biomed Eng 2012;40(8):1628-40.

104. McKeon-Fischer KD, Browe DP, Olabisi RM, Freeman JW. Poly(3,4ethylenedioxythiophene) nanoparticle and poly(varepsilon-caprolactone) electrospun scaffold characterization for skeletal muscle regeneration. J Biomed Mater Res A 2015;103(11):3633-41.

105. Martin R, Mullins ME, Zhao F, Qian Z. Electospinning 3D Scaffolds for use in Neural Tissue Engineering. MRS Proceedings[Online] 2015;1798.

106. Pawar K, Cummings BJ, Thomas A, Shea LD, Levine A, Pfaff S, Anderson AJ. Biomaterial bridges enable regeneration and re-entry of corticospinal tract axons into the caudal spinal cord after SCI: Association with recovery of forelimb function. Biomaterials 2015;65:1-12.

107. Nune M, Krishnan UM, Sethuraman S. PLGA nanofibers blended with designer self-assembling peptides for peripheral neural regeneration. Mater Sci Eng C Mater Biol Appl 2016;62:329-37. 
108. Moreno-Cortez IE, Alvarado-Castañeda A, Garcia-Gutierrez DF, Garcia-Gomez NA, Sepulveda-Guzman S, Garcia-Gutierrez DI. Core-shell PEDOT:PSS—PVP nanofibers containing PbS nanoparticles through coaxial electrospinning. Synthetic Metals 2016;220:255-262.

109. Jin L, Wang T, Feng Z-Q, Leach MK, Wu J, Mo S, Jiang Q. A facile approach for the fabrication of core-shell PEDOT nanofiber mats with superior mechanical properties and biocompatibility. Journal of Materials Chemistry B 2013;1(13):1818.

110. Kiristi M, Oksuz AU, Oksuz L, Ulusoy S. Electrospun chitosan/PEDOT nanofibers. Mater Sci Eng C Mater Biol Appl 2013;33(7):3845-50.

111. Lim CH. Production of Liquid Core-Polymer Shell Microcapsules. Houghton, Michigan: Michigan Technological University; 2011. 182 p.

112. Seo JM. Solution Property and Characteristic Study for Ultra Fine Fibers Via Electrospinning. Houghton, MI: Michigan Technological University; 2005. 


\section{Appendices}

\subsection{MRS Conference Proceedings: Electrospinning 3D Scaffolds for use in Neural Tissue Engineering}

Rachel Martin ${ }^{1}$, M. E. Mullins ${ }^{1}$, F. Zhao ${ }^{2}$, Zichen Qian $^{2}$

${ }^{1}$ Department of Chemical Engineering, Michigan Technological University, Houghton, MI

${ }^{2}$ Department of Biomedical Engineering, Michigan Technological University, Houghton, MI

\section{ABSTRACT}

Polymer nanofiber scaffolds for use in neural tissue engineering have been fabricated via electrospinning of poly-L-lactic acid (PLLA) directly onto a 3D printed support. Previously, the investigators have shown success in promoting the directed growth of neural axons on highly aligned PLLA substrates both in vitro and in vivo. However, one criticism of the earlier in vitro studies is that by spinning fibers on a flat, two-dimensional surface, the growth of the axons is restricted to one plane. Thus the axon-to-fiber attachment may not be the sole mechanism for aligning the growth of the axons along the fibers, and the channels between the fibers and the substrate could contribute to the results. Using 3D-printing, elevated or "bridge" spinning stages were made with supports at varying heights, allowing the fibers to be suspended 2 to $5 \mathrm{~mm}$ above the substrate surface in different configurations. This 3D structure promotes better access of in vitro cell cultures on the fibers to the growth media during incubation, 
reduces substrate effects, allows more degrees of freedom for axonal growth, and more closely simulates the growth environment found in vivo. Using these $3 \mathrm{D}$ stages, we have electrospun free-standing, highly-aligned pure PLLA fiber scaffolds. We are exploring spinning coaxial fibers with a PLLA sheath and a second core polymer. These coaxial fiber scaffold structures offer additional opportunities for in situ delivery of growth agents and/or electrical stimulation for improved axonal growth results.

\section{INTRODUCTION}

Previous research using highly aligned PLLA fibers spun on flat, 2-D coverslips proved successful during in vitro studies in aligning neural outgrowth of the axons from chick dorsal root ganglia (DRG)[1]. It was found that the axons grew along the aligned fibers in a parallel fashion, which is important if directed axonal outgrowth from neurons is to be achieved in vivo. In addition, in vivo rat studies were performed at the KennedyKrieger Institute of Johns Hopkins University[2]. A high percentage of spinal cord regeneration in rats who had suffered a surgically severed spine was observed. However, Hind Limb Motor Function (HLMF) tests produced lower scores than anticipated, indicating that the desired degree of neural reconnection was not achieved[2].

One criticism of the previous research is that the success of the in vitro studies may not be solely due to the alignment of the nanofibers, since the fibers were spun onto a flat surface, thus restricting the outgrowth of axons within a 2D plane. However, the nanofiber scaffolds used for the in vivo studies were constructed within a 3-dimensional tubular structure. In this configuration, in addition to axonal growth along the fibers, the axons may also grow outward from the plane of the fibers. If this phenomenon 
contributes to a significant decrease in the alignment of the axons in vivo, then the mixed results of the HLMF studies might be explained.

To address this hypothesis, we have devised a method for fabricating 3D tissue scaffolds. Via 3D printing, we have constructed a PLLA substrate or "stage" onto which we have electrospun PLLA nanofibers. The stages have been fabricated with support columns at various heights, and the fibers have been directly electrospun across the columns; thus the fibers are suspended at a specified distance from the surface of the stage. Subsequently, by replicating the previous in vitro studies with chick DRG cells, first on 2D coverslips as before, and then by using similarly prepared 3D "bridge" stages, the effect of the extra degrees of freedom on axonal outgrowth may be observed.

\section{EXPERIMENTAL}

\section{$\underline{\text { Electrospinning Methods }}$}

Our studies have employed a conventional electrospinning apparatus, as shown in the schematic and photograph in Figure 8-1. The principles of electrospinning from a polymer solution have been previously discussed in detail[3]. Our system also employs a rotating ground electrode or "wheel" for alignment of the fibers during collection from the spinning process. For the present work, either $6.5 \mathrm{wt} \%$ or $8 \mathrm{wt} \%$ PLLA in chloroform was used as the spinning solution. Samples were prepare by dissolving the desired weight of solid PLLA into a sample vial with $10 \mathrm{~mL}$ of chloroform and a stir bar. The vial was capped, sealed with Parafilm ${ }^{\circledR}$, and heated and mixed until the solution was homogenous (one to two hours). The solution was then drawn into a syringe fitted with a 16 gauge 
needle, and placed in a syringe pump. The pump flow rate was set based upon the PLLA concentration of the solution, but ranged between 0.8 and $1.7 \mathrm{~mL} / \mathrm{hr}$. Depending upon multiple variables, including the concentration of the solution, the humidity of the room and inside of the box, and the temperature of the room and inside the box, the flow rate was increased or lowered to achieve stable spinning. A higher flow rate was required for a more concentrated, viscous solution; whereas, a lower flow rate was required for less concentrated solutions. Tygon ${ }^{\circledR}$ tubing (I.D. $0.8 \mathrm{~mm}$ ) was connected to the syringe needle and the other end into the injection port. A 22 gauge "spinning" needle was attached to the injection port end of the tubing, and threaded through a specially fitted nozzle. The spinning needle was placed at a standard distance of $10 \mathrm{~cm}$ above a spinning aluminum wheel to allow sufficient distance to produce dry fibers. The collection wheel is attached to a variable speed motor set to $1250 \mathrm{rpm}$. The entire apparatus is placed inside a Plexiglas ${ }^{\circledR}$ box, allowing control of the spinning environment. Dry air can be pumped into the box to control the humidity, and other disturbances in the lab are less likely to affect the spinning process. A Glassman ${ }^{\circledR}$ high voltage power supply is connected to the spinning needle and set to between 15 and $25 \mathrm{kV}$. Halogen lamps were used to illuminate the box, and a video camera focused on the needle to allow a clear view of the spinning, and to record the results. We can then verify that a Taylor cone (Figure 8-2) is formed at the needle tip to ensure proper spinning is occurring and high quality fibers are spun.

When the apparatus is active, solution is pulled from the needle by the voltage difference between the charged needle and the grounded aluminum wheel as a thin fiber 1 to 4 microns thick. The fibers collect on the stages and flat coverslips that are attached to 
the rotating wheel. Spinning times varied, but usually lasted between 10 and 30 minutes in order to collect a dense enough fiber layer. Once spinning was complete, the sample stages were removed and optically imaged, then sterilized and set aside for the tissue culture studies.
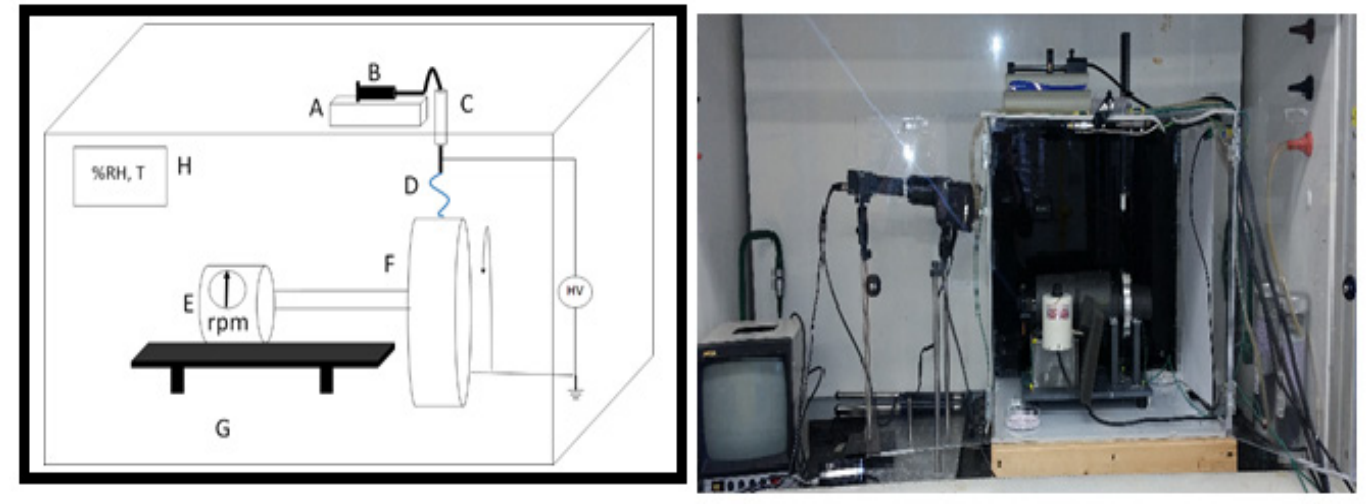

Figure 8-1: Left: A schematic of the electrospinning apparatus. (A) syringe pump, (B) syringe, (C) injector port, (D) needle with fiber strand production, (E) motor with speed control, (F) 6" aluminum wheel, (G) vibration table, (H) temperature/relative humidity meter, (I) humidity control with air flow, (J) light with high powered halogen bulbs, (K) camera, (L) TV monitor, (M) recorder. Right: Photo of spinning apparatus. 


\section{Electrospinning Stages}

Spinning stages were made on a 3D printer out of PLLA for compatibility with the fibers and the subsequent culture media. The initial design is simple, with perpendicular columns extending from the base of the stage. The column heights range from 2 to $4 \mathrm{~mm}$, to provide sufficient clearance between the fibers and the base, but not so high as to disturb the electrical field and air currents during the spinning process. Figure 8-3 shows photographs of a typical stage. The stages were adhered to the outer circumference of the aluminum wheel on the electrospinning apparatus using doublesided tape. The fibers are then directly spun across the columns and remain suspended above the surface of the stage. By simply changing the shape of the stage, we can thereby create a mandrel on which a variety of 3D shapes can then be spun directly, without additional manipulation or contamination.

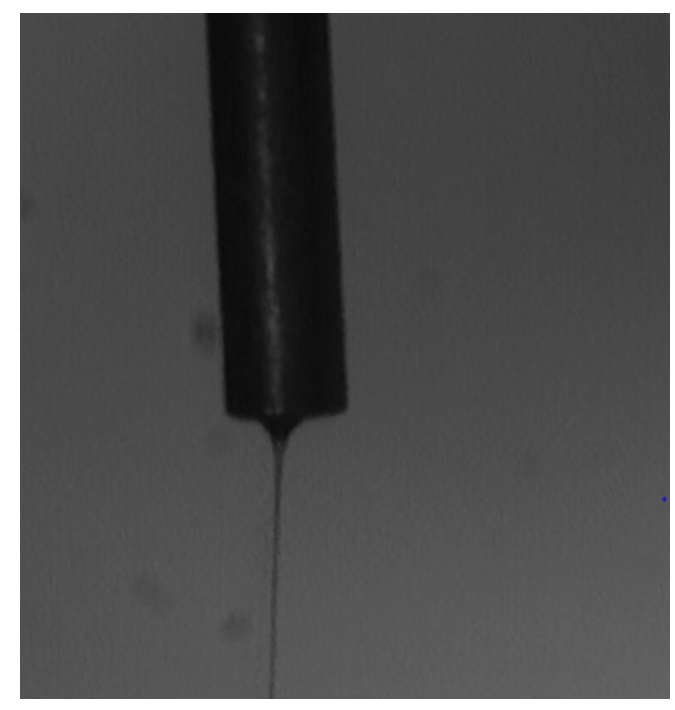

Figure 8-2: Video image of Taylor cone during spinning 

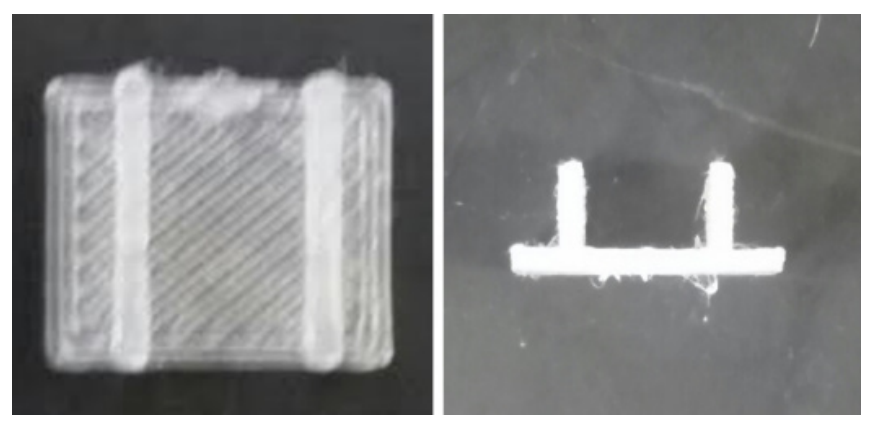

Figure 8-3: 3D printed polylactic acid stages. The dimensions of the base are $1 \mathrm{cmx} 1 \mathrm{~cm}$, height of the columns ranges from $3 \mathrm{~mm}$ to $4 \mathrm{~mm}$ in height. The stages are placed on the wheel and the fibers are spun across the columns so they are suspended above the base of the stage.

\section{DISCUSSION}

Images of the fiber scaffold samples, both on the 3D stages and on flat coverslips, were obtained using an optical microscope with a digital camera attachment to determine if they were suitable for the tissue culture studies. Characteristics including dryness, diameter, alignment and density of fiber mat were determined using the microscope. Figure 4 shows examples of the spinning results obtained for both flat coverslips and 3D stages obtained during the same electrospinning run (i.e. under the same process conditions, spinning time and solution concentration.) Here the typical fiber diameter was from 2 to $5 \mu \mathrm{m}$. 
Tissue cultures were performed using chick dorsal root ganglia (DRG) cells obtained from fertilized chick eggs. The eggs were incubated for 9 days and the DRG's harvested. The cells were seeded onto sterilized fiber samples and cultured for 5-7 days. The samples were then fluorescently stained and imaged. The cell harvesting and cultures procedures are discussed in detail elsewhere ${ }^{1}$. Figures 8-5A and 58-B show images obtained from 5 day cell cultures. In Figure 8-5C, a 7 day cell culture performed on fibers spun on a 3D stage is shown. The images in Figures $8-5 \mathrm{~B}$ and $8-5 \mathrm{C}$ show mixed results, with axons both following the fibers in some regions and jumping across the top to other areas of the sample. Figure 5 shows two images from 5 day cell cultures performed, one on a flat coverslip and one on a stage. As can be seen in Figure 8-5A, the axonal outgrowth of the nerve cell follows the axis of alignment of the PLLA fibers, which is very similar to what was observed previously ${ }^{1}$. On the other hand, in Figure $8-5 \mathrm{~B}$, the axonal outgrowth of the DRG seeded on the fibers is not as easily seen, but it appears as though the axons are still attempting to follow the fibers, but are not very closely aligned. A major aspect of this project was to determine if fibers could be spun onto not just 2D surfaces, but as free-standing fibers; and if the fibers could be aligned similarly to those spun on flat coverslips. As can be seen in Figure 8-4, we have successfully electrospun highly aligned, free standing fiber samples on the $3 \mathrm{D}$ stages comparable to the fiber samples spun on 2D surfaces. Figures 8-4A and 8-4B show fibers spun across the bridged structure which and appear very similar to the fibers spun on flat coverslips (Figures 8-4C-D) in size, density, and alignment. This is important because it allows us to perform tissue cultures on both supported and unsupported samples, thus providing information on the mechanisms for the aligned axonal growth. 


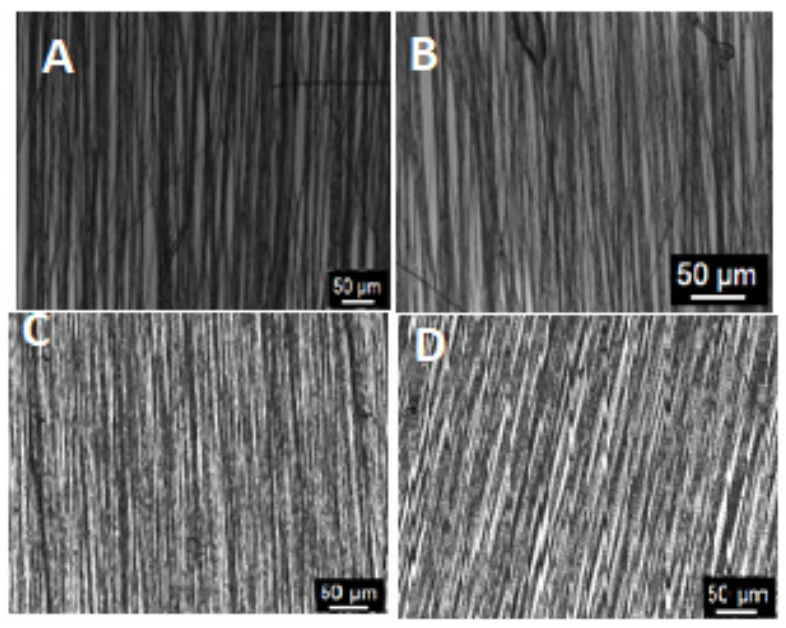

Figure 8-4: Optical microscopic images of the electrospun PLLA fibers. Spinning time was $23 \mathrm{~min}$ with a voltage of $17 \mathrm{KV}$ and a flow rate of $1.05 \mathrm{~mL} / \mathrm{hr}$. A and $\mathrm{B}$ show fibers spun on $3 D$ stages with columns at $4 \mathrm{~mm}$ column height. $C$ and $D$ show fibers spun on flat coverslips. The samples were obtained on 12/11/14. 

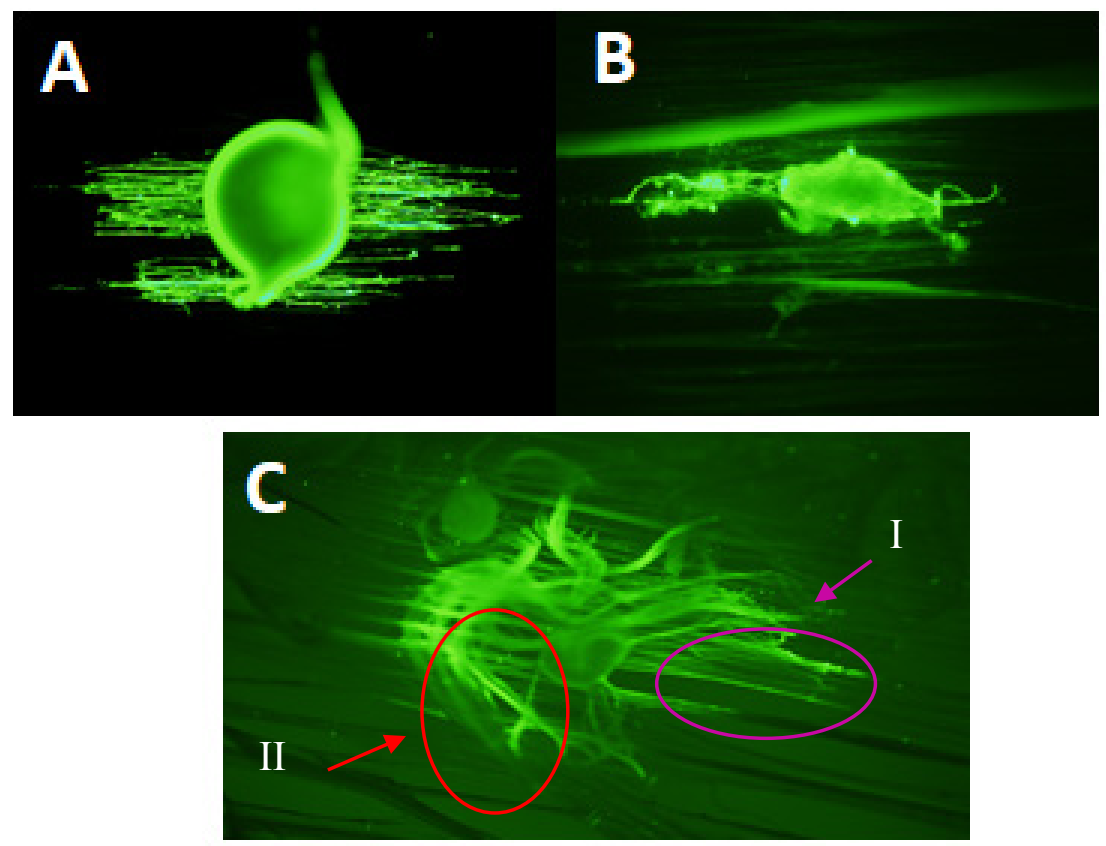

Figure 8-5: Fluorescent imaging of chick DRG cell cultures after 5 and 7 days. (A) shows a 5 day culture of a DRG performed on 3/19/15 seeded on fibers spun on a flat coverslip. The axons are growing straight outwards, along the alignment of the fibers. (B,C) 5 day cell culture performed on 3/19/15 and 7 day cell culture performed on 12/18/14. Both cells were seeded on stages. (B) The axons of the nerve cell seeded on the fibers spun on the stage still appear to be following the fibers, but it does not appear to have adhered very well. (C) The image is showing dual results. The righthand side of the cell (I) appears to have axons following the fibers. However, the lefthand side of the cell and the top of the cell (II) appear to have grown vertically and jumped to another area of the fiber network. Fiber conditions for the samples cultured on 3/19/15 are 8wet\% solution spun for 23 minutes on 12/11/14. 
Figures 8-5B-C show results from chick DRG studies performed on 3D stages. Initially, there was difficulty in seeding the cells on the elevated fibers, perhaps because of a lack of fiber density. If the fiber density was not sufficiently high, the DRGs may not attach or fall off. The DRGs that were well attached to the tissue scaffold exhibited a range of interesting axonal growth behavior. Figure 8-5A shows how the axons follow the alignment of the fibers when the fibers are spun on coverslips, which was similar to what was observed previously[1]. Figure 8-5B shows a 7 day culture on a 3D stage with different results. Groups of axons from the DRG appear to still be attempting to follow the alignment of the fibers. This grouping or clumping of axons during growth has been noted in the absence of aligned fibers or on PLLA films used as supports[1]. It appears in this image that the DRG did not adhere well, which indicates the extremely important effect of cell/axon adhesion in the cultures, and in the alignment of axonal growth with the fiber scaffold. Figure 8-5C shows dual results, where some of the axons appear to be following the alignment of the fibers, yet additional axonal growth is seen outside of the plane of the fiber scaffold. It appears that the axons, if not strongly attached to the fiber surface may not reliably grow in alignment with the fibers. Here some axons grow above or below the plane of the stage, into the plane, jump to a different part of the sample, or have limited extension. The axons on the left-hand side of the sample in 8-5C appear to exhibit all of these behaviors. More studies will be performed to determine the cause.

\section{FUTURE WORK}

In the next phase of our research, we are examining the effect of surface chemistry on cell adhesion. By enhancing the axonal adhesion to the PLLA fiber with 
the addition of surface modifiers, the alignment of axonal growth with the freestanding fibers may be improved. This may in turn, improve the success of the in vivo scaffold implants. Better adhesion might be achieved by using laminins, proteoglycans or similar substances that promote stronger axonal adhesion on the fiber surface, and observing the effect on axon alignment with the fibers. We will also explore electrospinning coaxial fibers. We would like to look at spinning fibers with a conductive polymer core and an insulating sheath. We will explore spinning fibers with a polypyrrole core and a PLLA sheath. We would also like to explore spinning fibers with a PEDOT core and a PLLA sheath, as PEDOT is known to have more stable conducting abilities than polypyrrole. We would also like to explore the ability to spin the fibers with the sheath containing pores so the axons have access to the conducting core. Once the new fibers are spun, in vitro studies using chick DRG cells will be performed, as well as new in vivo rat studies.

\section{CONCLUSIONS}

We have shown that consistent, 3D scaffolds can be manufactured using 3D printing, and fibers can be spun on these scaffolds. The fibers that are spun are of appropriate quality for cell cultures. We have also shown that cell cultures can be performed on these samples. However, the culture studies performed are not conclusive as to the driving force behind the axonal growth. More culture studies need to be performed in order to further determine the surface effects on axonal outgrowth and the mechanism of the outgrowth. The ability to manufacture 3D scaffolds allows for the freedom to spin samples of varying geometries. This may provide an advantage later in performing in vivo studies. We would like to explore the possibility of making new 3D 
structures for the spinning process, such as spinning conduits directly rather than rolling them as done previously[2].

\section{ACKNOWLEDGMENTS}

Thank you to Dr. Joshua Pierce of the Department of Materials Science and Engineering at Michigan Technological University providing the 3D stages. Thank you to Dr. Feng Zhao and Zichen Qian in the Department of Biomedical Engineering at Michigan Technological University for helping with the cell cultures. Thank you to the Department of Chemical Engineering at Michigan Technological University for providing the funding for this project.

\section{REFERENCES}

1. Wang, Han Bing. Mullins, Michael E. Creation of highly aligned electrospun poly-L-lactic acid fibers for nerve regeneration applications. Journal of Neural Engineering, December (2008).

2. Andres Hurtado, Ryan J. Gilbert, Han B. Wang, Jared M. Cregg, Michael E. Mullins, Martin Oudega. Three-dimensional scaffolds, methods for fabricating the same, and methods of treating a peripheral nerve or spinal cord injury. US Patent US20130110138 A1, (2013)

3. Wang, Han Bing. Development of an implantable multilayer 3D scaffold consisting of aligned electrospun fibers for neuronal regeneration. Michigan Technological University (2008). 
This Agreement between Rachel Martin ("You") and Cambridge University Press ("Cambridge University Press") consists of your license details and the terms and conditions provided by Cambridge University Press and Copyright Clearance Center.

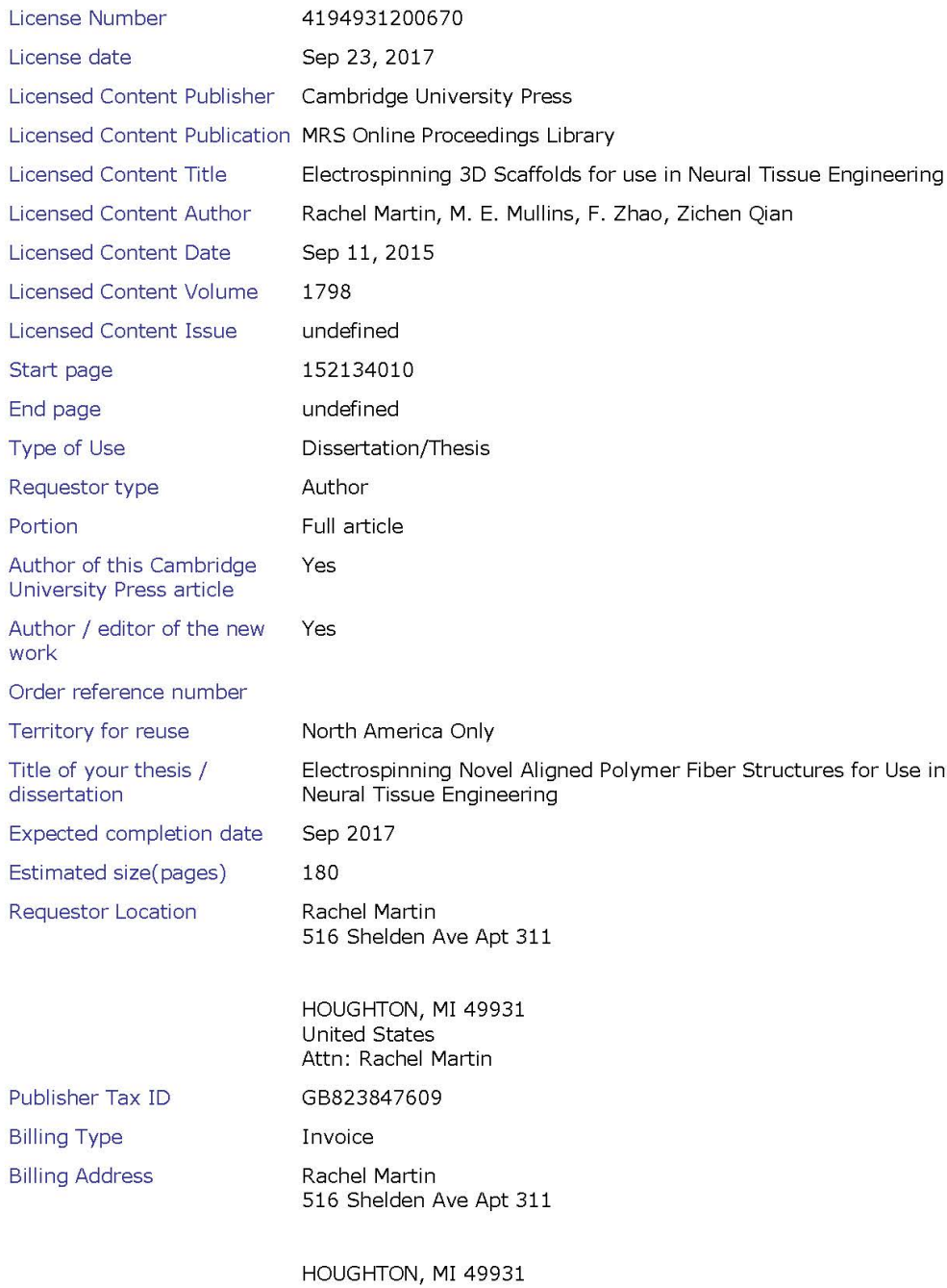


$9 / 26 / 2017$

Rights Link Printable License

United States

Attn: Rachel Martin

Total

0.00 USD

Terms and Conditions

TERMS \& CONDITIONS

Cambridge University Press grants the Licensee permission on a non-exclusive nontransferable basis to reproduce, make available or otherwise use the Licensed content 'Content' in the named territory 'Territory' for the purpose listed 'the Use' on Page 1 of this Agreement subject to the following terms and conditions.

1. The License is limited to the permission granted and the Content detailed herein and does not extend to any other permission or content.

2. Cambridge gives no warranty or indemnity in respect of any third-party copyright material included in the Content, for which the Licensee should seek separate permission clearance.

3. The integrity of the Content must be ensured.

4. The License does extend to any edition published specifically for the use of handicapped or reading-impaired individuals.

5. The Licensee shall provide a prominent acknowledgement in the following format: author/s, title of article, name of journal, volume number, issue number, page references, , reproduced with permission.

Other terms and conditions:

$\mathrm{v} 1.0$

Questions? customercare@copyright.com or +1-855-239-3415 (toll free in the US) or +1-978-646-2777 


\subsection{Additional Optical Microscopy and FE-SEM Images Showing}

\section{Core-Sheath Structure}

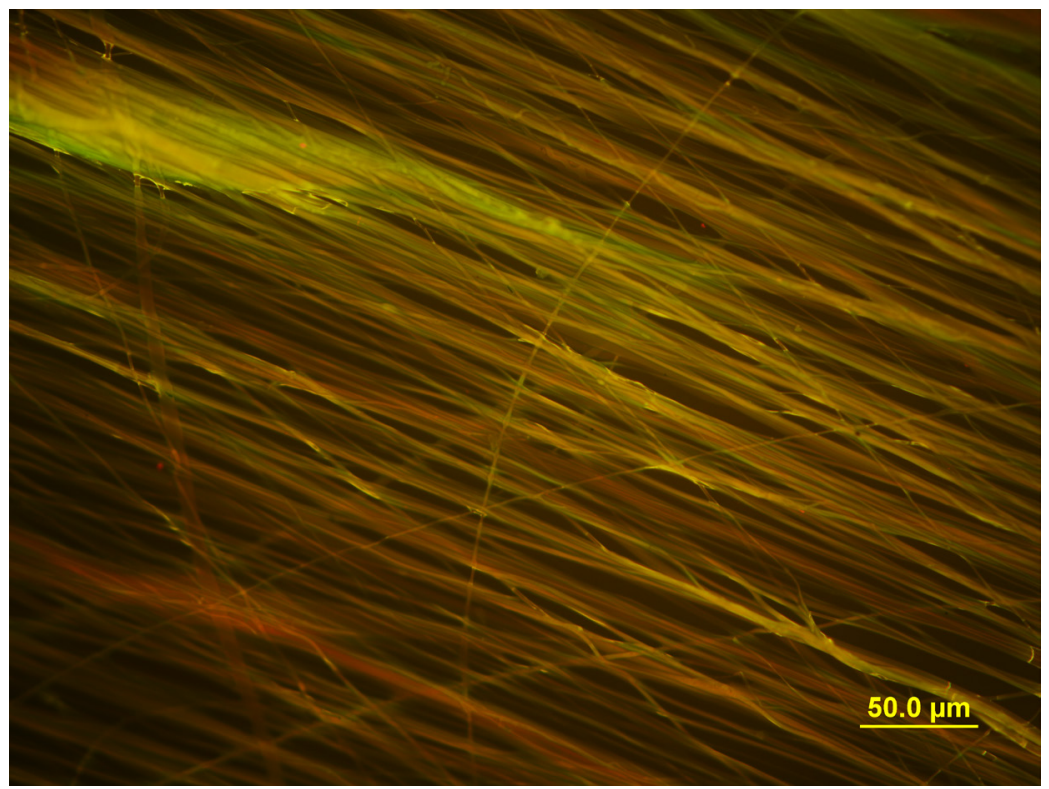

Figure 8-6: Yellow overlay created from red and green fluorescence images obtained from a coaxial fiber sample with PEDOT:DBSA nanoparticles in the core. Spun at 2500rpm wheel rotation speed and $16 G$ outer needle. 


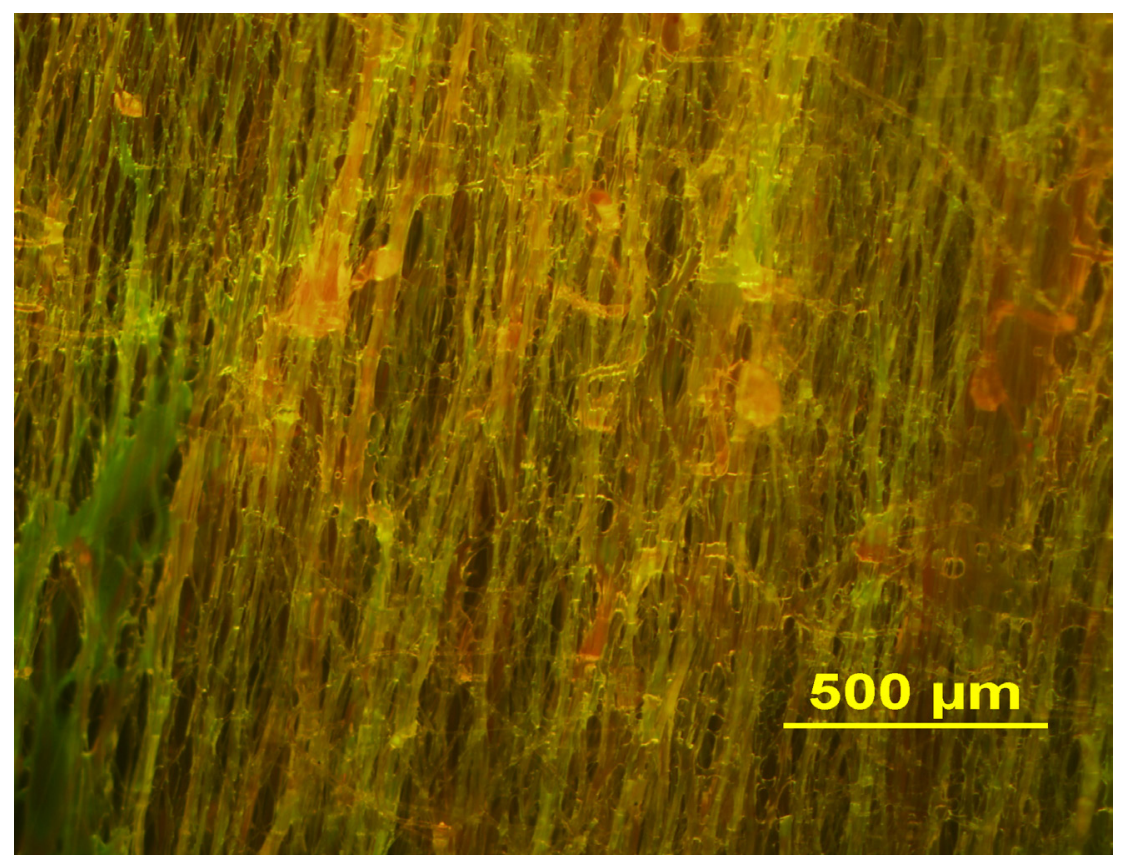

Figure 8-7: Yellow overlay created from red and green fluorescent images from a coaxial fiber sample with PEDOT:PSS in the core. Spun at 2250rpm wheel rotation speed and $16 G$ outer needle. 


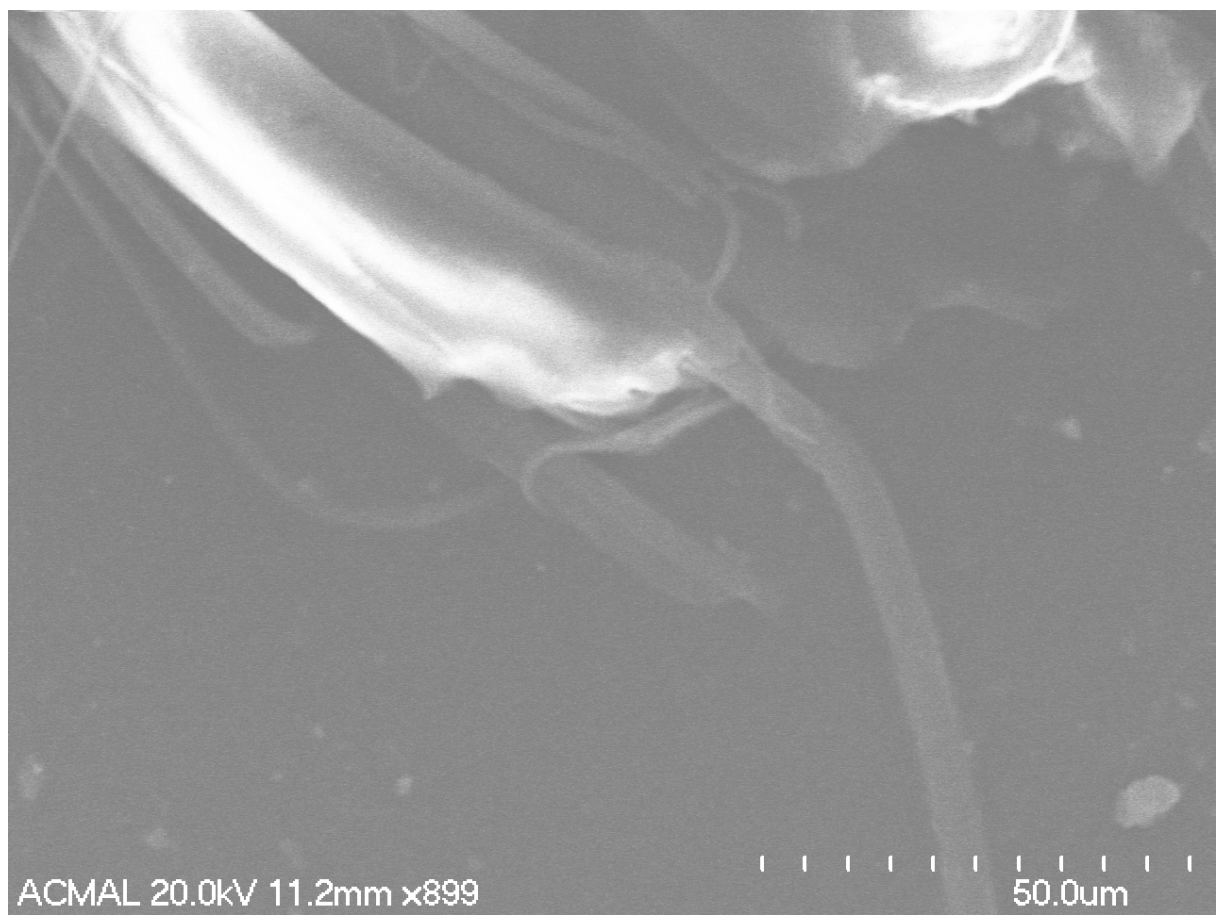

Figure 8-8: FE-SEM image obtained showing core-sheath structure. The core is pulled out of the sheath and is shown to be conductive. Apparant brightening of the sheath does not occur for the core fiber strand. 


\subsection{Copyright Clearance for Images used in Chapter 2}

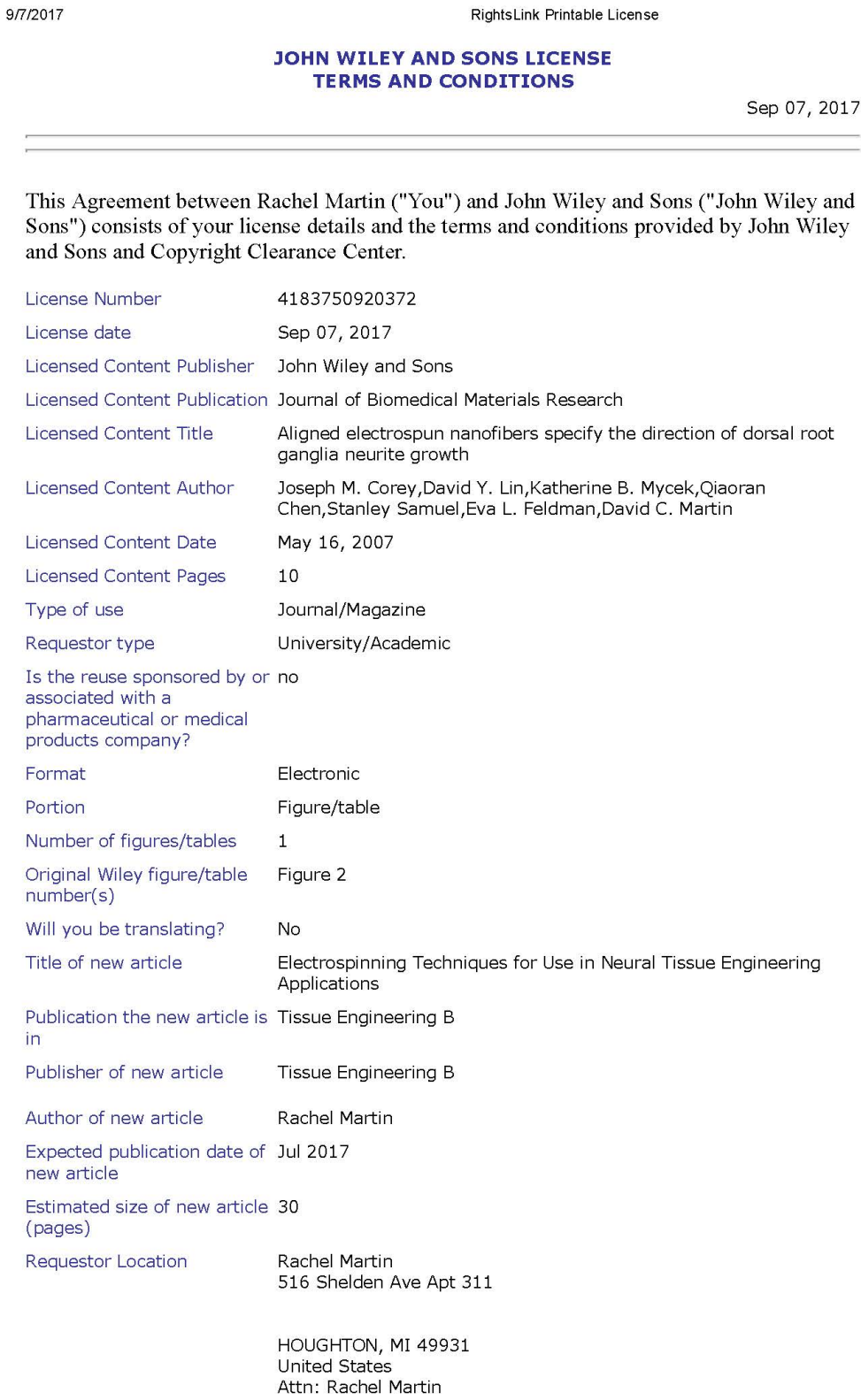




$\begin{array}{ll}9 / 7 / 2017 & \\ \text { Publisher Tax ID } & \text { EU826007151 } \\ \text { Billing Type } & \text { Invoice } \\ \text { Billing Address } & \text { Rachel Martin } \\ & 516 \text { Shelden Ave Apt 311 } \\ & \\ & \text { HOUGHTON, MI 49931 } \\ & \text { United States } \\ & \text { Attn: Rachel Martin } \\ \text { Total } & 0.00 \text { USD } \\ \text { Terms and Conditions } & \end{array}$

\section{TERMS AND CONDITIONS}

This copyrighted material is owned by or exclusively licensed to John Wiley \& Sons, Inc. or one of its group companies (each a"Wiley Company") or handled on behalf of a society with which a Wiley Company has exclusive publishing rights in relation to a particular work (collectively "WILEY"). By clicking "accept" in connection with completing this licensing transaction, you agree that the following terms and conditions apply to this transaction (along with the billing and payment terms and conditions established by the Copyright Clearance Center Inc., ("CCC's Billing and Payment terms and conditions"), at the time that you opened your RightsLink account (these are available at any time at http://mvaccount.copvright.com).

\section{Terms and Conditions}

- The materials you have requested permission to reproduce or reuse (the "Wiley Materials") are protected by copyright.

- You are hereby granted a personal, non-exclusive, non-sub licensable (on a standalone basis), non-transferable, worldwide, limited license to reproduce the Wilev Materials for the purpose specified in the licensing process. This license, and any CONTENT (PDF or image file) purchased as part of your order, is for a one-time use only and limited to any maximum distribution number specified in the license. The first instance of republication or reuse granted by this license must be completed within two years of the date of the grant of this license (although copies prepared before the end date may be distributed thereafter). The Wiley Materials shall not be used in any other manner or for any other purpose, beyond what is granted in the license. Permission is granted subject to an appropriate acknowledgement given to the author, title of the material/book/journal and the publisher. You shall also duplicate the copyright notice that appears in the Wiley publication in your use of the Wiley Material. Permission is also granted on the understanding that nowhere in the text is a previously published source acknowledged for all or part of this Wiley Material. Any third party content is expressly excluded from this permission.

- With respect to the Wiley Materials, all rights are reserved. Except as expressly granted by the terms of the license, no part of the Wiley Materials may be copied, modified, adapted (except for minor reformatting required by the new Publication), translated, reproduced, transferred or distributed, in any form or by any means, and no derivative works may be made based on the Wiley Materials without the prior permission of the respective copyright owner.For STM Signatory Publishers clearing permission under the terms of the STM Permissions Guidelines only, the terms of the license are extended to include subsequent editions and for editions in other languages, provided such editions are for the work as a whole in situ and does not involve the separate exploitation of the permitted figures or extracts, 
You may not alter, remove or suppress in any manner any copyright, trademark or other notices displayed by the Wiley Materials. You may not license, rent, sell, loan, lease, pledge, offer as security, transfer or assign the Wiley Materials on a stand-alone basis, or any of the rights granted to you hereunder to any other person.

- The Wiley Materials and all of the intellectual property rights therein shall at all times remain the exclusive property of John Wiley \& Sons Inc, the Wiley Companies, or their respective licensors, and your interest therein is only that of having possession of and the right to reproduce the Wiley Materials pursuant to Section 2 herein during the continuance of this Agreement. You agree that you own no right, title or interest in or to the Wiley Materials or any of the intellectual property rights therein. You shall have no rights hereunder other than the license as provided for above in Section 2. No right, license or interest to any trademark, trade name, service mark or other branding ("Marks") of WILEY or its licensors is granted hereunder, and you agree that you shall not assert any such right, license or interest with respect thereto

- NEITHER WILEY NOR ITS LICENSORS MAKES ANY WARRANTY OR REPRESENTATION OF ANY KIND TO YOU OR ANY THIRD PARTY, EXPRESS, IMPLIED OR STATUTORY, WITH RESPECT TO THE MATERIALS OR THE ACCURACY OF ANY INFORMATION CONTAINED IN THE MATERIALS, INCLUDING, WITHOUT LIMITATION, ANY IMPLIED WARRANTY OF MERCHANTABILITY, ACCURACY, SATISFACTORY QUALITY, FITNESS FOR A PARTICULAR PURPOSE, USABILITY, INTEGRATION OR NON-INFRINGEMENT AND ALL SUCH WARRANTIES ARE HEREBY EXCLUDED BY WILEY AND ITS LICENSORS AND WAIVED BY YOU.

- WILEY shall have the right to terminate this Agreement immediately upon breach of this Agreement by you.

- You shall indemnify, defend and hold harmless WILEY, its Licensors and their respective directors, officers, agents and employees, from and against any actual or threatened claims, demands, causes of action or proceedings arising from any breach of this Agreement by you.

- IN NO EVENT SHALL WILEY OR ITS LICENSORS BE LIABLE TO YOU OR ANY OTHER PARTY OR ANY OTHER PERSON OR ENTITY FOR ANY SPECIAL, CONSEQUENTIAL, INCIDENTAL, INDIRECT, EXEMPLARY OR PUNITIVE DAMAGES, HOWEVER CAUSED, ARISING OUT OF OR IN CONNECTION WITH THE DOWNLOADING, PROVISIONING, VIEWING OR USE OF THE MATERIALS REGARDLESS OF THE FORM OF ACTION, WHETHER FOR BREACH OF CONTRACT, BREACH OF WARRANTY, TORT, NEGLIGENCE, INFRINGEMENT OR OTHERWISE (INCLUDING, WITHOUT LIMITATION, DAMAGES BASED ON LOSS OF PROFITS, DATA, FILES, USE, BUSINESS OPPORTUNITY OR CLAIMS OF THIRD PARTIES), AND WHETHER OR NOT THE PARTY HAS BEEN ADVISED OF THE POSSIBILITY OF SUCH DAMAGES. THIS LIMITATION SHALL APPLY NOTWITHSTANDING ANY FAILURE OF ESSENTIAL PURPOSE OF ANY LIMITED REMEDY PROVIDED HEREIN.

- Should any provision of this Agreement be held by a court of competent jurisdiction to be illegal, invalid, or unenforceable, that provision shall be deemed amended to achieve as nearly as possible the same economic effect as the original provision, and the legality, validity and enforceability of the remaining provisions of this Agreement 
shall not be affected or impaired thereby.

- The failure of either party to enforce any term or condition of this Agreement shall not constitute a waiver of either party's right to enforce each and every term and condition of this Agreement. No breach under this agreement shall be deemed waived or excused by either party unless such waiver or consent is in writing signed by the party granting such waiver or consent. The waiver by or consent of a party to a breach of any provision of this Agreement shall not operate or be construed as a waiver of or consent to any other or subsequent breach by such other party.

- This Agreement may not be assigned (including by operation of law or otherwise) by you without WILEY's prior written consent.

- Any fee required for this permission shall be non-refundable after thirty (30) days from receipt by the $\mathrm{CCC}$.

- These terms and conditions together with CCC's Billing and Payment terms and conditions (which are incorporated herein) form the entire agreement between you and WILEY concerning this licensing transaction and (in the absence of fraud) supersedes all prior agreements and representations of the parties, oral or written. This Agreement may not be amended except in writing signed by both parties. This Agreement shall be binding upon and inure to the benefit of the parties' successors, legal representatives, and authorized assigns.

- In the event of any conflict between your obligations established by these terms and conditions and those established by CCC's Billing and Payment terms and conditions, these terms and conditions shall prevail.

- WILEY expressly reserves all rights not specifically granted in the combination of (i) the license details provided by you and accepted in the course of this licensing transaction, (ii) these terms and conditions and (iii) CCC's Billing and Payment terms and conditions.

- This Agreement will be void if the Type of Use, Format, Circulation, or Requestor Type was misrepresented during the licensing process.

- This Agreement shall be governed by and construed in accordance with the laws of the State of New York, USA, without regards to such state's conflict of law rules. Any legal action, suit or proceeding arising out of or relating to these Terms and Conditions or the breach thereof shall be instituted in a court of competent jurisdiction in New York County in the State of New York in the United States of America and each party hereby consents and submits to the personal jurisdiction of such court, waives any objection to venue in such court and consents to service of process by registered or certified mail, return receipt requested, at the last known address of such party.

\section{WILEY OPEN ACCESS TERMS AND CONDITIONS}

Wiley Publishes Open Access Articles in fully Open Access Journals and in Subscription journals offering Online Open. Although most of the fully Open Access journals publish open access articles under the terms of the Creative Commons Attribution (CC BY) License only, the subscription journals and a few of the Open Access Journals offer a choice of Creative Commons Licenses. The license type is clearly identified on the article.

The Creative Commons Attribution License 
The Creative Commons Attribution License (CC-BY) allows users to copy, distribute and transmit an article, adapt the article and make commercial use of the article. The CC-BY license permits commercial and non-

Creative Commons Attribution Non-Commercial License

The Creative Commons Attribution Non-Commercial (CC-BY-NC)License permits use, distribution and reproduction in any medium, provided the original work is properly cited and is not used for commercial purposes.(see below)

\section{Creative Commons Attribution-Non-Commercial-NoDerivs License}

The Creative Commons Attribution Non-Commercial-NoDerivs License (CC-BY-NC-ND) permits use, distribution and reproduction in any medium, provided the original work is properly cited, is not used for commercial purposes and no modifications or adaptations are made. (see below)

Use by commercial "for-profit" organizations

Use of Wiley Open Access articles for commercial, promotional, or marketing purposes requires further explicit permission from Wiley and will be subject to a fee.

Further details can be found on Wiley Online Library

http://olabout.wilev.com/WilevCDA/Section/id-410895.html

Other Terms and Conditions:

v1.10 Last updated September 2015

Questions? customercare@copyright.com or +1-855-239-3415 (toll free in the US) or +1-978-646-2777. 


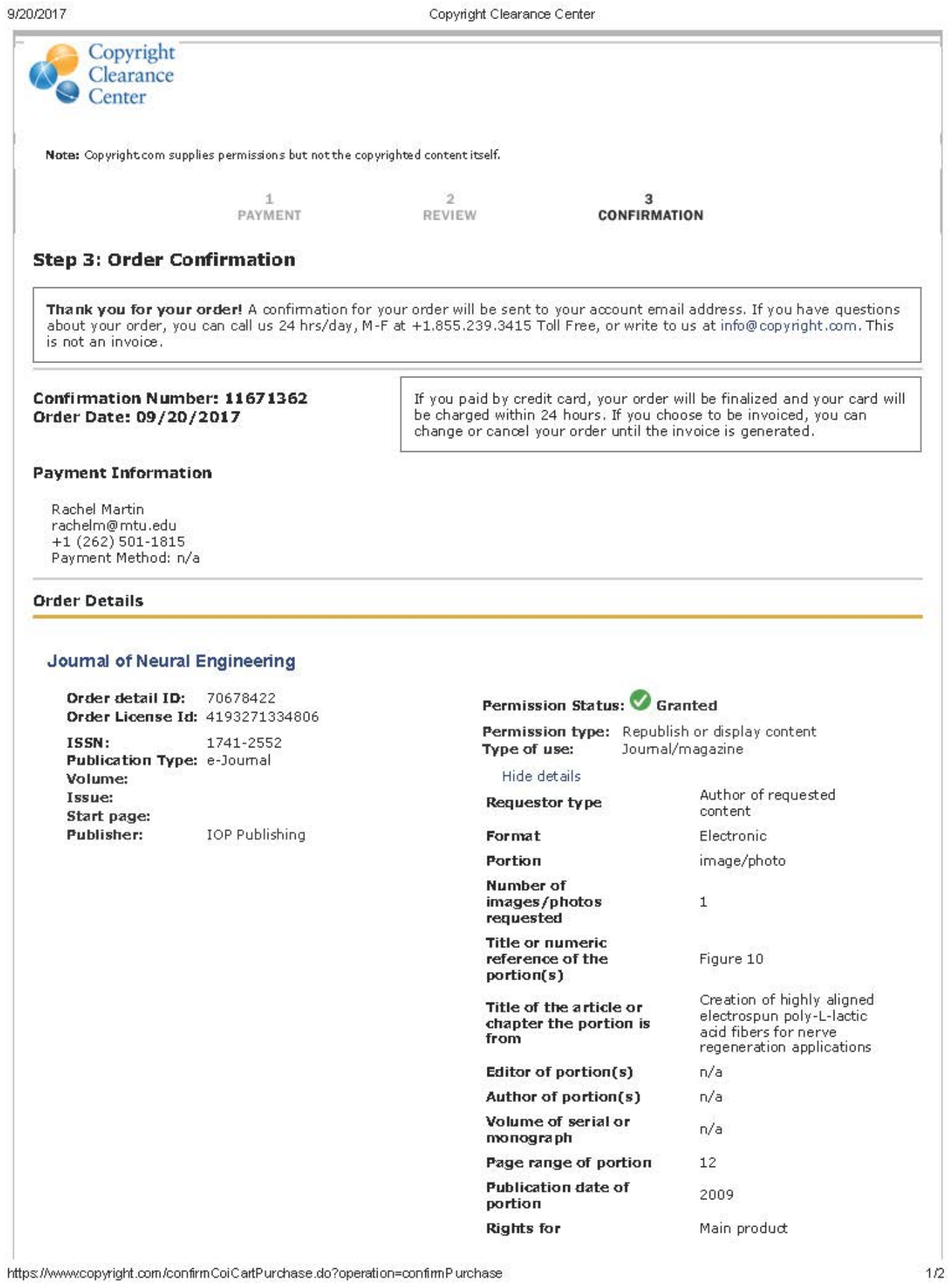




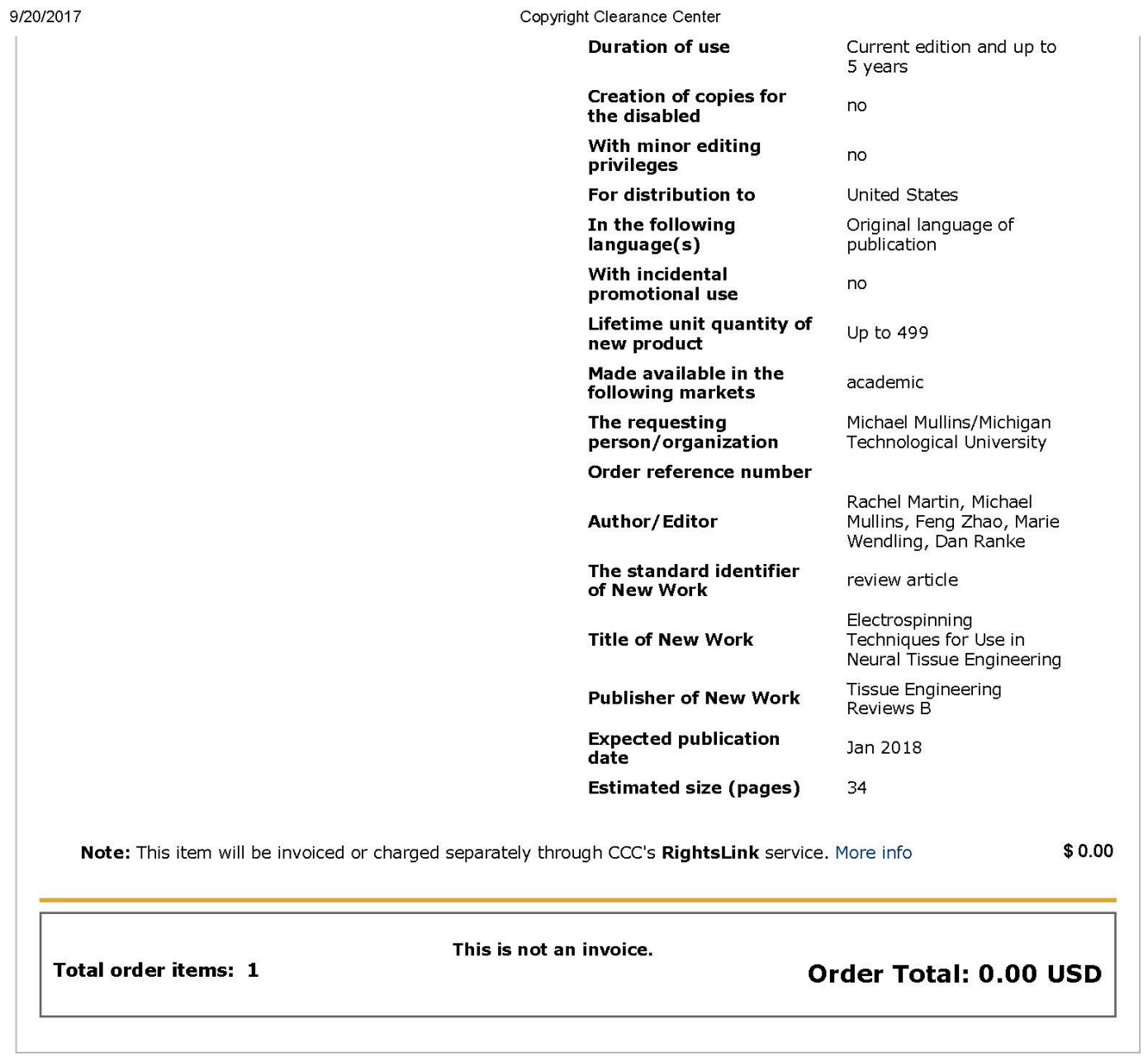




\section{JOHN WILEY AND SONS LICENSE}

TERMS AND CONDITIONS

This Agreement between Rachel Martin ("You") and John Wiley and Sons ("John Wiley and Sons") consists of your license details and the terms and conditions provided by John Wiley and Sons and Copyright Clearance Center.

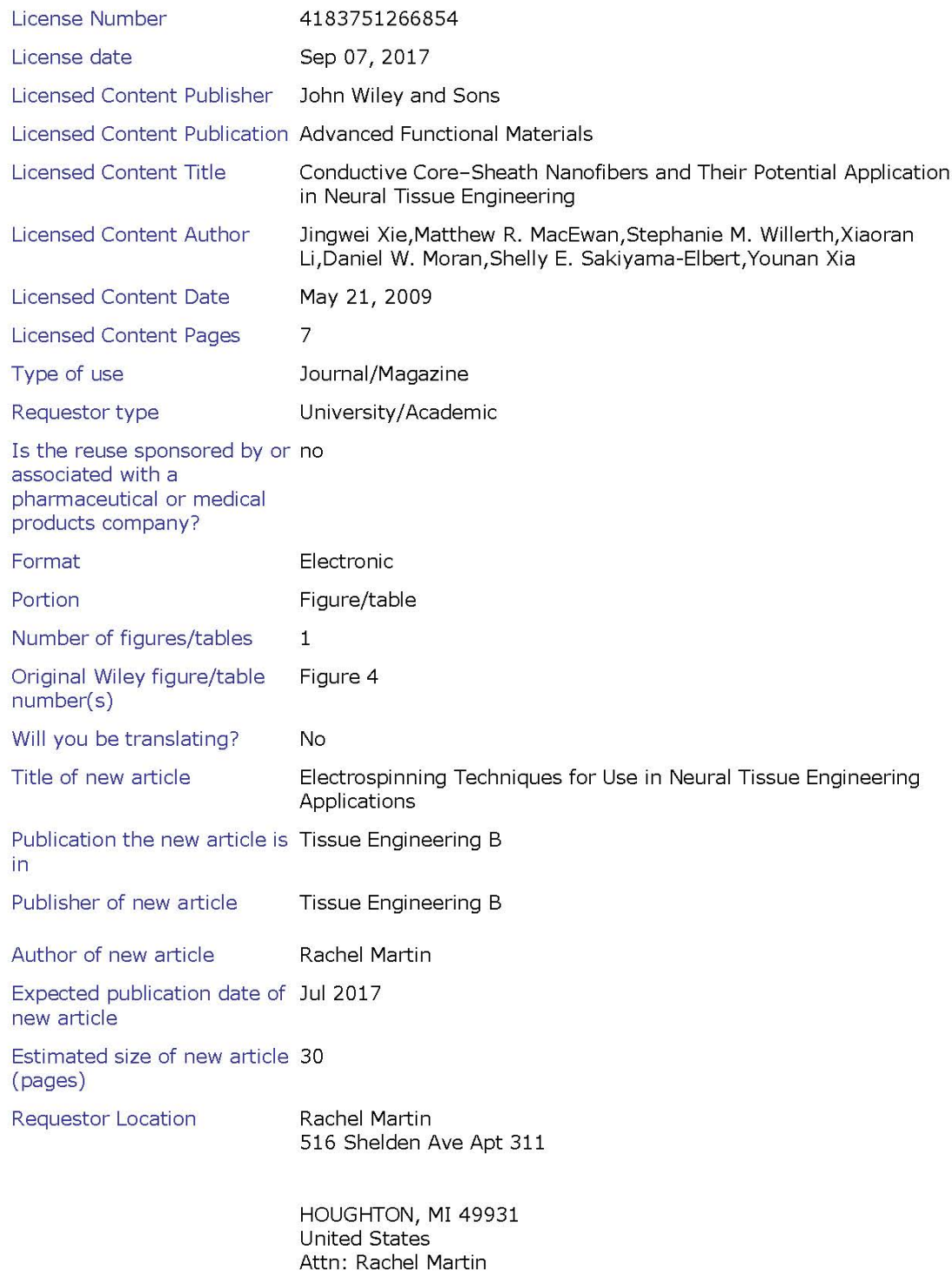




$\begin{array}{ll}9 / 7 / 2017 & \\ \text { Publisher Tax ID } & \text { EU826007151 } \\ \text { Billing Type } & \text { Invoice } \\ \text { Billing Address } & \text { Rachel Martin } \\ & 516 \text { Shelden Ave Apt 311 } \\ & \\ & \text { HOUGHTON, MI 49931 } \\ & \text { United States } \\ & \text { Attn: Rachel Martin } \\ \text { Total } & 0.00 \text { USD } \\ \text { Terms and Conditions } & \end{array}$

\section{TERMS AND CONDITIONS}

This copyrighted material is owned by or exclusively licensed to John Wiley \& Sons, Inc. or one of its group companies (each a"Wiley Company") or handled on behalf of a society with which a Wiley Company has exclusive publishing rights in relation to a particular work (collectively "WILEY"). By clicking "accept" in connection with completing this licensing transaction, you agree that the following terms and conditions apply to this transaction (along with the billing and payment terms and conditions established by the Copyright Clearance Center Inc., ("CCC's Billing and Payment terms and conditions"), at the time that you opened your RightsLink account (these are available at any time at http://mvaccount.copvright.com).

\section{Terms and Conditions}

- The materials you have requested permission to reproduce or reuse (the "Wiley Materials") are protected by copyright.

- You are hereby granted a personal, non-exclusive, non-sub licensable (on a standalone basis), non-transferable, worldwide, limited license to reproduce the Wilev Materials for the purpose specified in the licensing process. This license, and any CONTENT (PDF or image file) purchased as part of your order, is for a one-time use only and limited to any maximum distribution number specified in the license. The first instance of republication or reuse granted by this license must be completed within two years of the date of the grant of this license (although copies prepared before the end date may be distributed thereafter). The Wiley Materials shall not be used in any other manner or for any other purpose, beyond what is granted in the license. Permission is granted subject to an appropriate acknowledgement given to the author, title of the material/book/journal and the publisher. You shall also duplicate the copyright notice that appears in the Wiley publication in your use of the Wiley Material. Permission is also granted on the understanding that nowhere in the text is a previously published source acknowledged for all or part of this Wiley Material. Any third party content is expressly excluded from this permission.

- With respect to the Wiley Materials, all rights are reserved. Except as expressly granted by the terms of the license, no part of the Wiley Materials may be copied, modified, adapted (except for minor reformatting required by the new Publication), translated, reproduced, transferred or distributed, in any form or by any means, and no derivative works may be made based on the Wiley Materials without the prior permission of the respective copyright owner.For STM Signatory Publishers clearing permission under the terms of the STM Permissions Guidelines only, the terms of the license are extended to include subsequent editions and for editions in other languages, provided such editions are for the work as a whole in situ and does not involve the separate exploitation of the permitted figures or extracts, 
You may not alter, remove or suppress in any manner any copyright, trademark or other notices displayed by the Wiley Materials. You may not license, rent, sell, loan, lease, pledge, offer as security, transfer or assign the Wiley Materials on a stand-alone basis, or any of the rights granted to you hereunder to any other person.

- The Wiley Materials and all of the intellectual property rights therein shall at all times remain the exclusive property of John Wiley \& Sons Inc, the Wiley Companies, or their respective licensors, and your interest therein is only that of having possession of and the right to reproduce the Wiley Materials pursuant to Section 2 herein during the continuance of this Agreement. You agree that you own no right, title or interest in or to the Wiley Materials or any of the intellectual property rights therein. You shall have no rights hereunder other than the license as provided for above in Section 2. No right, license or interest to any trademark, trade name, service mark or other branding ("Marks") of WILEY or its licensors is granted hereunder, and you agree that you shall not assert any such right, license or interest with respect thereto

- NEITHER WILEY NOR ITS LICENSORS MAKES ANY WARRANTY OR REPRESENTATION OF ANY KIND TO YOU OR ANY THIRD PARTY, EXPRESS, IMPLIED OR STATUTORY, WITH RESPECT TO THE MATERIALS OR THE ACCURACY OF ANY INFORMATION CONTAINED IN THE MATERIALS, INCLUDING, WITHOUT LIMITATION, ANY IMPLIED WARRANTY OF MERCHANTABILITY, ACCURACY, SATISFACTORY QUALITY, FITNESS FOR A PARTICULAR PURPOSE, USABILITY, INTEGRATION OR NON-INFRINGEMENT AND ALL SUCH WARRANTIES ARE HEREBY EXCLUDED BY WILEY AND ITS LICENSORS AND WAIVED BY YOU.

- WILEY shall have the right to terminate this Agreement immediately upon breach of this Agreement by you.

- You shall indemnify, defend and hold harmless WILEY, its Licensors and their respective directors, officers, agents and employees, from and against any actual or threatened claims, demands, causes of action or proceedings arising from any breach of this Agreement by you.

- IN NO EVENT SHALL WILEY OR ITS LICENSORS BE LIABLE TO YOU OR ANY OTHER PARTY OR ANY OTHER PERSON OR ENTITY FOR ANY SPECIAL, CONSEQUENTIAL, INCIDENTAL, INDIRECT, EXEMPLARY OR PUNITIVE DAMAGES, HOWEVER CAUSED, ARISING OUT OF OR IN CONNECTION WITH THE DOWNLOADING, PROVISIONING, VIEWING OR USE OF THE MATERIALS REGARDLESS OF THE FORM OF ACTION, WHETHER FOR BREACH OF CONTRACT, BREACH OF WARRANTY, TORT, NEGLIGENCE, INFRINGEMENT OR OTHERWISE (INCLUDING, WITHOUT LIMITATION, DAMAGES BASED ON LOSS OF PROFITS, DATA, FILES, USE, BUSINESS OPPORTUNITY OR CLAIMS OF THIRD PARTIES), AND WHETHER OR NOT THE PARTY HAS BEEN ADVISED OF THE POSSIBILITY OF SUCH DAMAGES. THIS LIMITATION SHALL APPLY NOTWITHSTANDING ANY FAILURE OF ESSENTIAL PURPOSE OF ANY LIMITED REMEDY PROVIDED HEREIN.

- Should any provision of this Agreement be held by a court of competent jurisdiction to be illegal, invalid, or unenforceable, that provision shall be deemed amended to achieve as nearly as possible the same economic effect as the original provision, and the legality, validity and enforceability of the remaining provisions of this Agreement 
shall not be affected or impaired thereby.

- The failure of either party to enforce any term or condition of this Agreement shall not constitute a waiver of either party's right to enforce each and every term and condition of this Agreement. No breach under this agreement shall be deemed waived or excused by either party unless such waiver or consent is in writing signed by the party granting such waiver or consent. The waiver by or consent of a party to a breach of any provision of this Agreement shall not operate or be construed as a waiver of or consent to any other or subsequent breach by such other party.

- This Agreement may not be assigned (including by operation of law or otherwise) by you without WILEY's prior written consent.

- Any fee required for this permission shall be non-refundable after thirty (30) days from receipt by the $\mathrm{CCC}$.

- These terms and conditions together with CCC's Billing and Payment terms and conditions (which are incorporated herein) form the entire agreement between you and WILEY concerning this licensing transaction and (in the absence of fraud) supersedes all prior agreements and representations of the parties, oral or written. This Agreement may not be amended except in writing signed by both parties. This Agreement shall be binding upon and inure to the benefit of the parties' successors, legal representatives, and authorized assigns.

- In the event of any conflict between your obligations established by these terms and conditions and those established by CCC's Billing and Payment terms and conditions, these terms and conditions shall prevail.

- WILEY expressly reserves all rights not specifically granted in the combination of (i) the license details provided by you and accepted in the course of this licensing transaction, (ii) these terms and conditions and (iii) CCC's Billing and Payment terms and conditions.

- This Agreement will be void if the Type of Use, Format, Circulation, or Requestor Type was misrepresented during the licensing process.

- This Agreement shall be governed by and construed in accordance with the laws of the State of New York, USA, without regards to such state's conflict of law rules. Any legal action, suit or proceeding arising out of or relating to these Terms and Conditions or the breach thereof shall be instituted in a court of competent jurisdiction in New York County in the State of New York in the United States of America and each party hereby consents and submits to the personal jurisdiction of such court, waives any objection to venue in such court and consents to service of process by registered or certified mail, return receipt requested, at the last known address of such party.

\section{WILEY OPEN ACCESS TERMS AND CONDITIONS}

Wiley Publishes Open Access Articles in fully Open Access Journals and in Subscription journals offering Online Open. Although most of the fully Open Access journals publish open access articles under the terms of the Creative Commons Attribution (CC BY) License only, the subscription journals and a few of the Open Access Journals offer a choice of Creative Commons Licenses. The license type is clearly identified on the article.

The Creative Commons Attribution License 
The Creative Commons Attribution License (CC-BY) allows users to copy, distribute and transmit an article, adapt the article and make commercial use of the article. The CC-BY license permits commercial and non-

Creative Commons Attribution Non-Commercial License

The Creative Commons Attribution Non-Commercial (CC-BY-NC)License permits use, distribution and reproduction in any medium, provided the original work is properly cited and is not used for commercial purposes.(see below)

\section{Creative Commons Attribution-Non-Commercial-NoDerivs License}

The Creative Commons Attribution Non-Commercial-NoDerivs License (CC-BY-NC-ND) permits use, distribution and reproduction in any medium, provided the original work is properly cited, is not used for commercial purposes and no modifications or adaptations are made. (see below)

Use by commercial "for-profit" organizations

Use of Wiley Open Access articles for commercial, promotional, or marketing purposes requires further explicit permission from Wiley and will be subject to a fee.

Further details can be found on Wiley Online Library

http://olabout.wilev.com/WilevCDA/Section/id-410895.html

Other Terms and Conditions:

v1.10 Last updated September 2015

Questions? customercare@copyright.com or +1-855-239-3415 (toll free in the US) or +1-978-646-2777. 